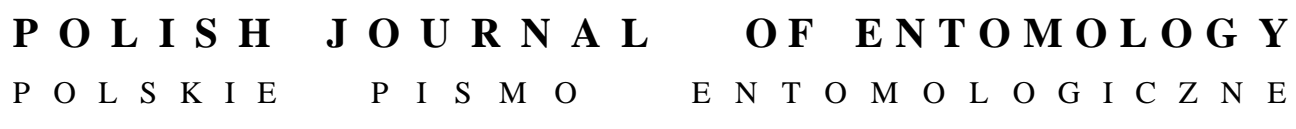

VOL. 85: 27-92

Lublin

30 March 2016

DOI: $10.1515 /$ pjen-2016-0003

\title{
Natural islands and habitat islands as refuges of vegetation cover and wild bees. The case of the Lednica Landscape Park in western Poland
}

\author{
JÓZEF BANASZAK ${ }^{1 *}$, HALINA RATYŃSKA ${ }^{2}$ \\ ${ }^{1}$ Department of Ecology, Institute of Biology and Environmental Protection, Kazimierz \\ Wielki University, Ossolińskich Av. 12, 85-093 Bydgoszcz, Poland \\ ${ }^{2}$ Department of Botany, Institute of Biology and Environmental Protection, Kazimierz \\ Wielki University, Ossolińskich Av. 12, 85-093 Bydgoszcz, Poland
}

\begin{abstract}
The study has contributed to the identification of the apifauna of central Wielkopolska. The study identified 161 bee species, accounting for $34.2 \%$ of the Polish bee fauna. The highest contribution (28.7\% of the fauna) comes from four species, namely Andrena haemorrhoa, A. helvola, Evylaeus calceatus and Osmia rufa, while Bombus terrestris and Evylaeus pauxillus are two subdominants. The assemblages of Apiformes in the study area are characterised by a significant contribution of spring-associated species, which is probably an effect of the presence of numerous willow thickets offering abundant host plants (mainly Salix sp. div.). Both the islands and the surroundings of the lake have a unique species composition, and there are differences in the proportions of the individual dominant species. The overall abundance of bees varies greatly, with mean seasonal density figures on Ostrów Lednicki Island being more than twice as high as that on the mainland grassland, with a distinct predominance of bumblebees. The exceptional richness of Apiformes, including bumblebees, on Ostrów Lednicki should be regarded as the basis for treating this island as a life refuge for bumblebees and including it and its environs in the list of sites of Community importance (SCI). A simultaneous study of the vegetation cover contributed significant data on the vascular plant flora and plant communities of the Lednica Landscape Park. For example, it was the first such investigation of Mewia Island. The study revealed the importance of marginal habitats (natural islands and habitat islands) for the preservation of protected and endangered plant species and plant communities receding from an agricultural landscape.
\end{abstract}

KEY WORDS: vascular plant flora, plant communities, Hymenoptera, Apoidea, Apiformes, bees, wild bees, habitat island, natural island.

* Corresponding author: lednica@ukw.edu.pl 


\section{INTRODUCTION}

The Lednica Landscape Park (LLP) is located in central Wielkopolska, approximately 30 $\mathrm{km}$ east of Poznań. It was established in 1988 to protect the nationally unique land around Lake Lednica, which was the cradle of Polish statehood. Abounding in monuments of the past and in an only slightly transformed postglacial landscape, it is an area of historical importance for Poles. It reached its prime in the $10^{\text {th }}$ and $11^{\text {th }}$ centuries $\mathrm{AD}$, when Ostrów Lednicki Island, the biggest of the four islands, housed the headquarters of Prince Mieszko I. Material remnants of those times are the ruins of immense stone edifices within the former stronghold: the Prince's palace (palatium) communicating with a chapel and the socalled second church. The discovery of baptismal pools in the chapel suggests that this was the very place where the baptism of Poland took place.

The Landscape Park occupies an area of 4854 hectares and has the shape of a belt approximately $10 \mathrm{~km}$ in length and a mean width of approximately $3 \mathrm{~km}$. The Park's protective buffer zone has a mean width of $1.5 \mathrm{~km}$, running near the Park's border only at its northern and southern ends (Fig. 1). The terrain of the LLP is formed by an undulating moraine plateau at an altitude of 110-115 m above sea level. The axis of the Park is Lake Lednica, which is $7300 \mathrm{~m}$ long and has an area of almost 340 ha. The maximum width of the lake is nearly $825 \mathrm{~m}$, and the maximum depth is $15.1 \mathrm{~m}$. The shoreline is indented. There are five islands on the lake, the largest of which is Ostrów Lednicki (7.5 ha in area). The other islands are Ledniczka Island and Mewia Island (or Ptasia Island) and two nameless islets. The lake's shores are treeless and, except for the south-western shore, they are tall. The River Główna flows out of the lake's southern end.

The vegetation cover of the Lednica Landscape Park is almost treeless and quite monotonous as a result of numerous settlements and intensive agricultural use since the early Middle Ages. Early medieval palynological data indicate the nearly total treelessness of both Ostrów Lednicki Island and the environs of Lake Lednica. Today, only the northern end of the lake is surrounded by a small forest (TOBOLSKI 1991, CHOJNACKA \& RASZKA 2007). Archaeological studies have revealed 257 plant taxa that, one thousand years ago, formed the phytocoenoses of both natural communities and those variously transformed by human management (POLCYN 2000). The contemporary vegetation cover of the LLP has been investigated, but the data are incomplete (CELKA 1998, 1999, POLCYN 2000, partly RATYŃSKA 2003).

The LLP has not been studied previously with regard to its bee fauna (Apiformes). Published work includes only minor contributions relating to certain species associated with clay buildings (BANASZAK 1995) and papers announcing the research described in the present paper (BANASZAK 1996, 1998, 2000). The presence of the currently rare species Anthophora plagiata (ILLIGER, 1806) has been reported by BANASZAK (2005). 


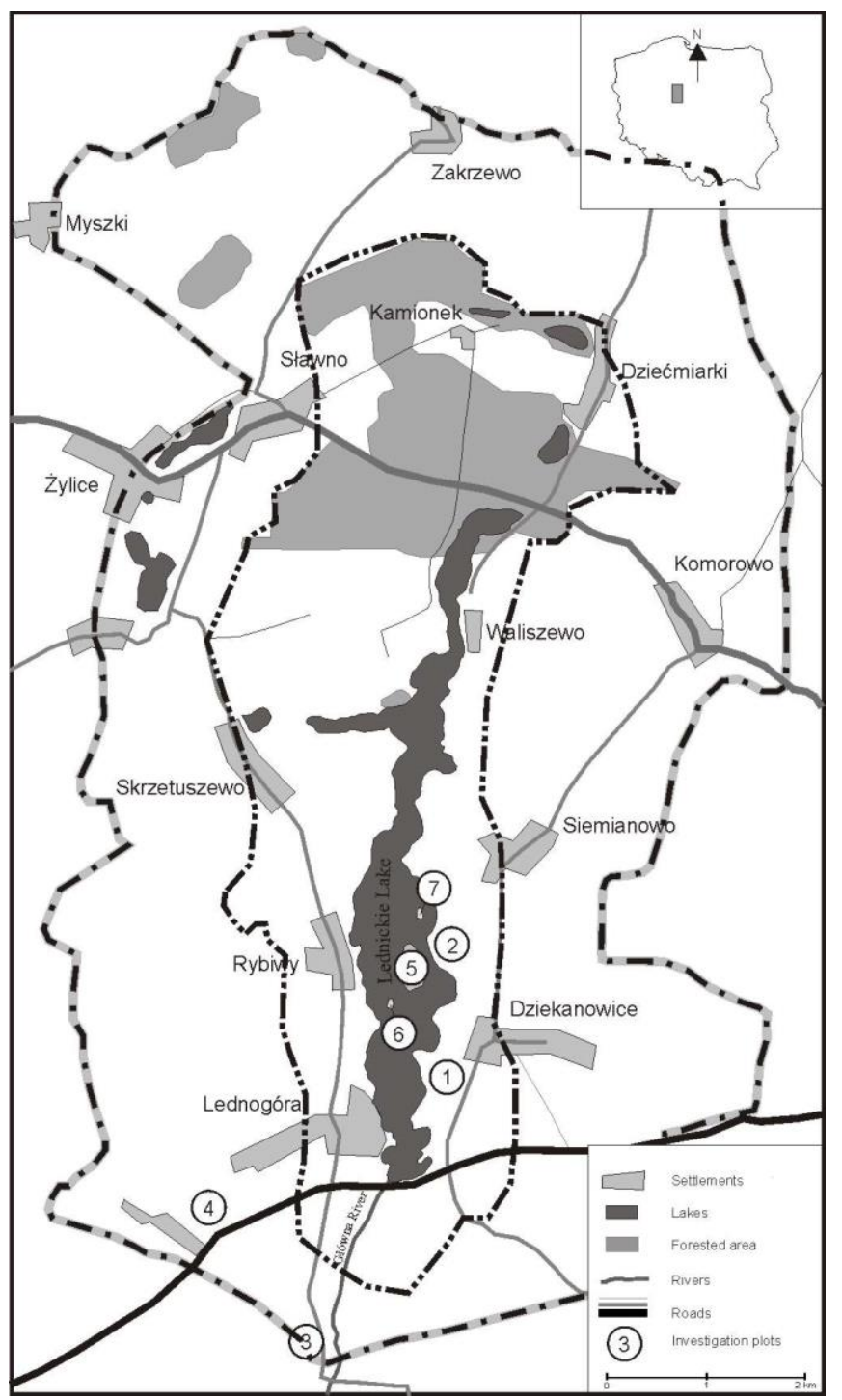

Fig. 1. Lednicki Landscape Park and locaton of study sites $(1$ - Large Heritage Park, 2 - Small Heritage Park, 3 - Posłonkowe Hill, 4 - Moraczewskie Hill, 5 - Ostrów Lednicki Island, $6-$ Ledniczka Island, 7 - Mewia Island.

Considering that the LLP area has a well-documented history of early human activity in terms of both settlement and agriculture, it appeared to be an ideal location for studying pollinating insects in an agricultural landscape.

The research project aimed to assess the species diversity of Apiformes, their dominance patterns and phenology in various habitat types represented by the islands on Lake Lednica and small marginal habitats (habitat islands) within the complex of agricultural fields. A detailed assessment of the flora and vegetation cover of the study sites 
contributes to a better identification of the food resources of Apiformes and adds to our knowledge about the vegetation cover in the Lednica Landscape Park. We would also like to give our heartfelt thanks to the then Director of the Museum of the First Piasts at Lednica, Andrzej KASZUBKIEWICZ, for making this study possible, and first and foremost, for providing technical and financial assistance in collecting materials during the several years of fieldwork. Thanks are also due to our colleagues Olga SONDEJ MSc, Amelia LEWANDOWSKA MSc and Piotr SZEFER MSc for their technical assistance.

Some of the materials investigated in our studies were obtained by the late, sorely missed, Roman DORUCHOWSKI (1965-2012) of the Museum of the First Piasts at Lednica. We should like to express our immense gratitude for his dedication and conscientious assistance. We dedicate this paper to his memory.

\section{STUDY AREA AND METHODS}

The dominant feature of the landscape of the Park is the presence of extensive agrocoenoses, with lower-lying areas being occupied by dried-out meadows and fens, while the higher ground is covered by arable fields. There is a small area of woodland only in the northern part of the Park. Its stands are of various ages and are clearly dominated by introduced pine.

The terrain in the Park is formed by an undulating morainic plateau lying at $110-120 \mathrm{~m}$ above sea level. The higher ground is covered by brown and loess soils, whereas the lowest-lying areas have muck or peat formations. In higher-lying spots there are chernozem soils. Local mounds have poorly developed soils, called arenosols (DRZYMAŁA \& MOCEK 1994).

A peculiarity of this historic area is the presence of numerous fortified settlements with rich grassland vegetation that is attractive to insects as a food resource and nesting site. The vascular flora of the fortified settlements accounts for half ( 283 species) of the species composition of the entire flora of the LLP (CELKA 1998).

Climatically, the LLP occupies an intermediate position between the diminishing features of subatlantic and subcontinental climates (ТовOLSKI 1991). The long-term mean precipitation is about $550 \mathrm{~mm}$, and the mean temperature is slightly above $8^{\circ} \mathrm{C}$. The coldest month is January, with a mean temperature of $-2.2^{\circ} \mathrm{C}$ and an absolute minimum of $-28.0^{\circ} \mathrm{C}$. The highest temperatures have been recorded in July, with a mean of $18.0^{\circ} \mathrm{C}$ and a maximum of $38.2^{\circ} \mathrm{C}$. According to the regionalisation by Woś (1999), the LLP is classified within climatic region XV (Central Wielkopolska), the largest in Poland. Compared to other areas, it is characterised by relatively frequent days of very warm but cloudy weather as well as a considerable frequency of very cold days with ground frost and 
snow. The inflow of continental air in summer leads to droughts. Black frost in spring caused by the dominant arctic air at this time of the year is a very undesirable feature (WoŚ 2003).

The study sites are three of the islands on Lake Lednica (Ostrów Lednicki, Ledniczka and Mewia), largely covered with woodland, and habitat islands - clusters of persistent semi-natural vegetation (meadows and pastures) or wooded areas (the Small Heritage Park, the Large Heritage Park, Moraczewskie Hill, Posłonkowe Hill) within the predominantly arable landscape. The study sites were selected for their diversity as habitats (from the potential biotope of a fertile alder forest to a mesic coniferous forest) and type of management, which is reflected in the current vegetation cover (from forest communities to ruderal plants).

The field studies of the flora were conducted between 1996 and 2013. Each of the seven sites was treated as a complex of plant communities (TÜXEN 1973, SOLON 1983, RATYŃSKA 2003). Only the Large Heritage Park, because of the substantial habitat differences and type of management, was divided into an area with historical buildings and accompanying arable land, and a steep escarpment supporting thermophilous grassland vegetation. Within these divisions, the plant communities were recorded together with the spontaneous vascular flora, and the abundance was estimated according to the Braun-Blanquet scale as modified by BARKMAN (1964). The nomenclature of plant communities and vascular plants follows the list given by RATYŃSKA et al. (2010). Works by the same authors were also used to assess the conservation status and surface area of phytocoenoses, and to classify them with regard to their response to anthropogenic impacts. The spectrum of geographical and historical groups and life-forms was analysed (JACKOWIAK 1990). Types of natural habitats were also distinguished, including priority types requiring protection under the Natura 2000 scheme (Ordinance of the Minister of 13 April 2010), and legally protected species (Ordinance of the Minister of 20 January 2012). The conservation status of vascular plants was assessed after MIREK et al. (2006) for Poland as a whole and according to JACKOWIAK et al. (2007) for the region. The details of the vascular plant species are given in Table 1 and of the plant communities in Table 2 . The vegetation cover of the various study sites are compared in Table 3. 


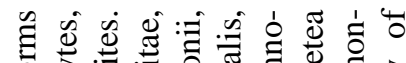

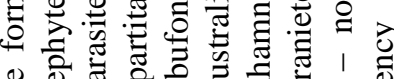

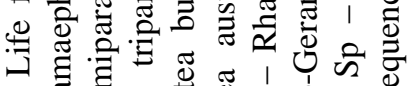

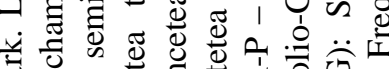

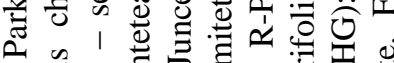
。

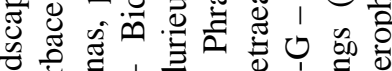
焉 \% ।

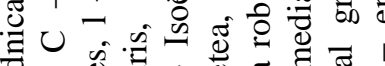

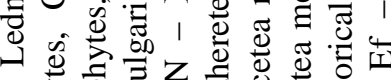

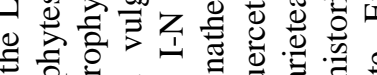

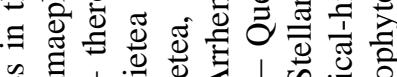

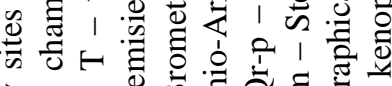

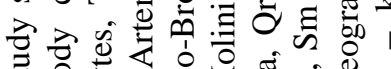

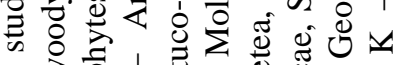

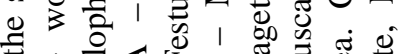

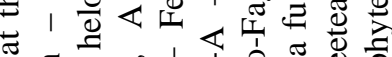

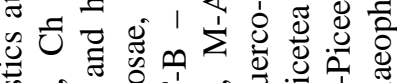

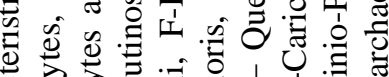

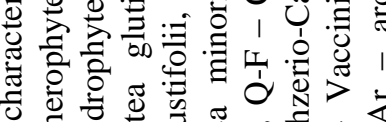
ए

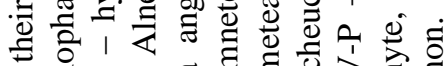
즌

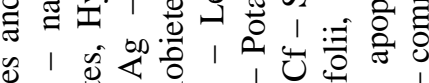
क

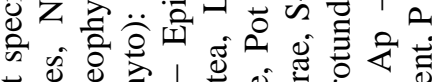

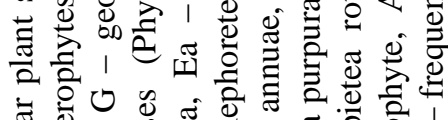

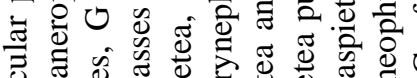

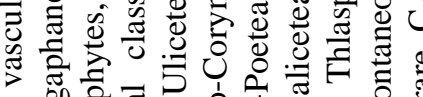

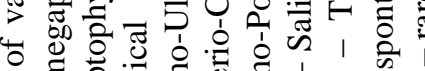
勿

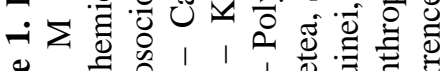

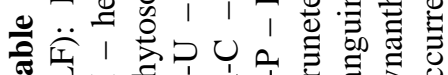

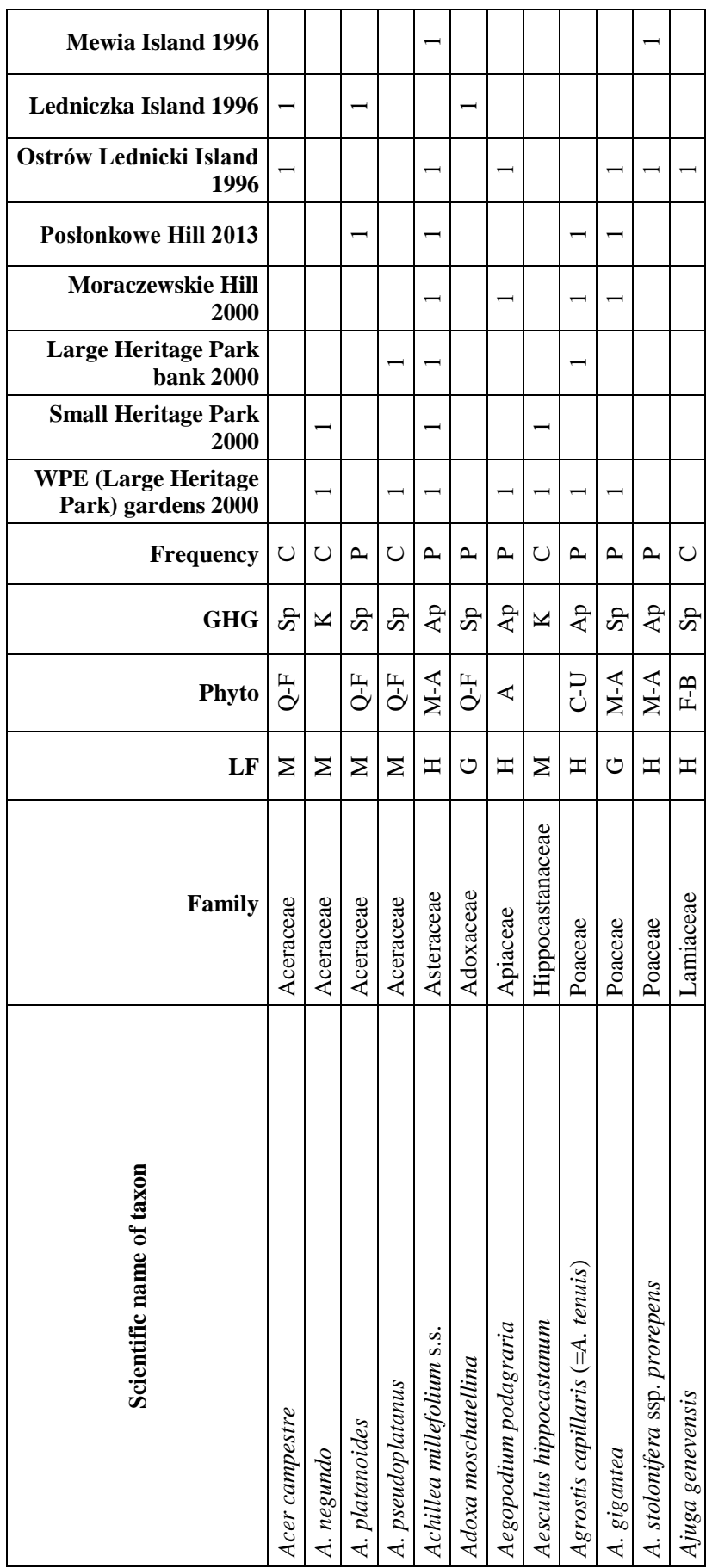




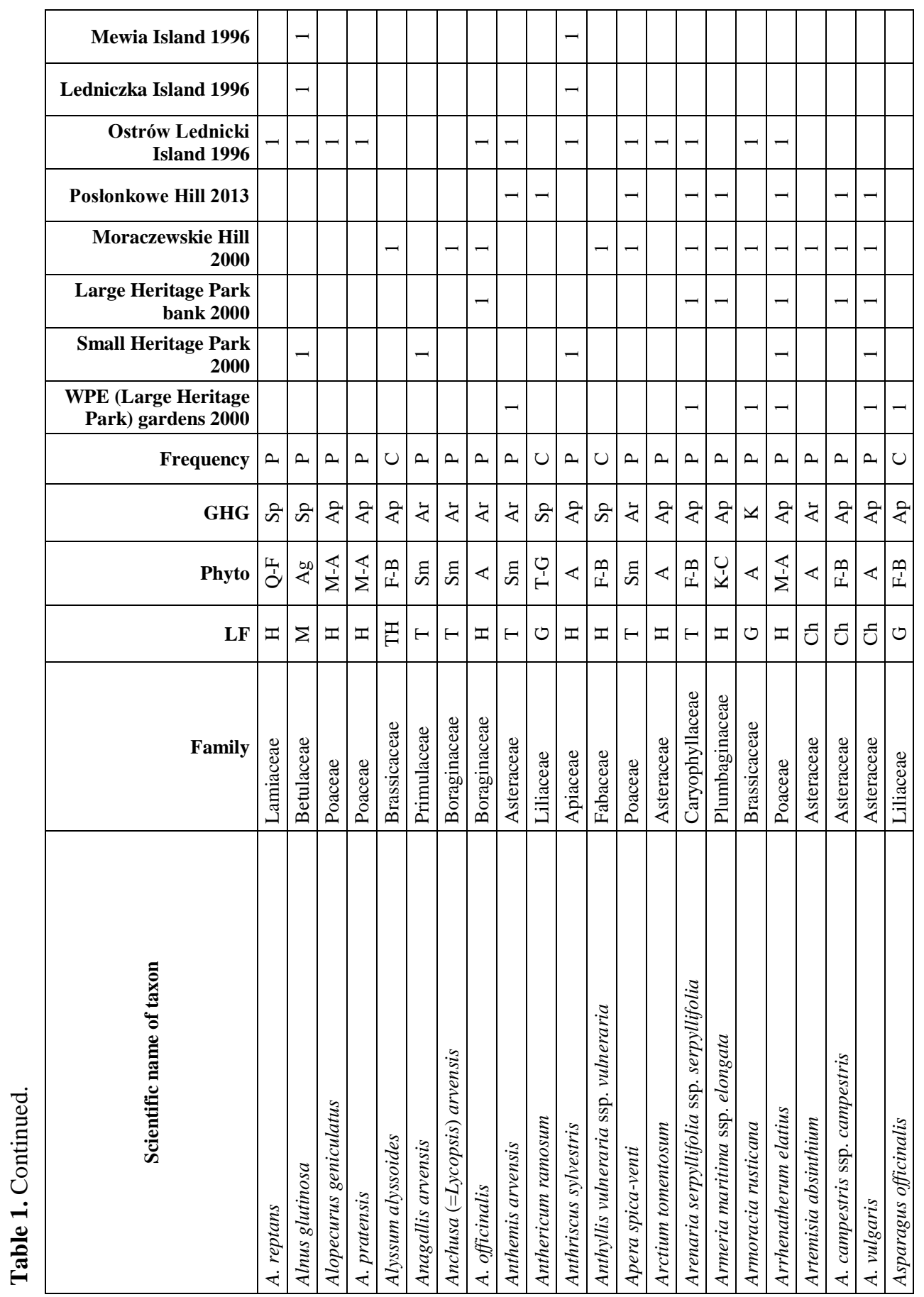




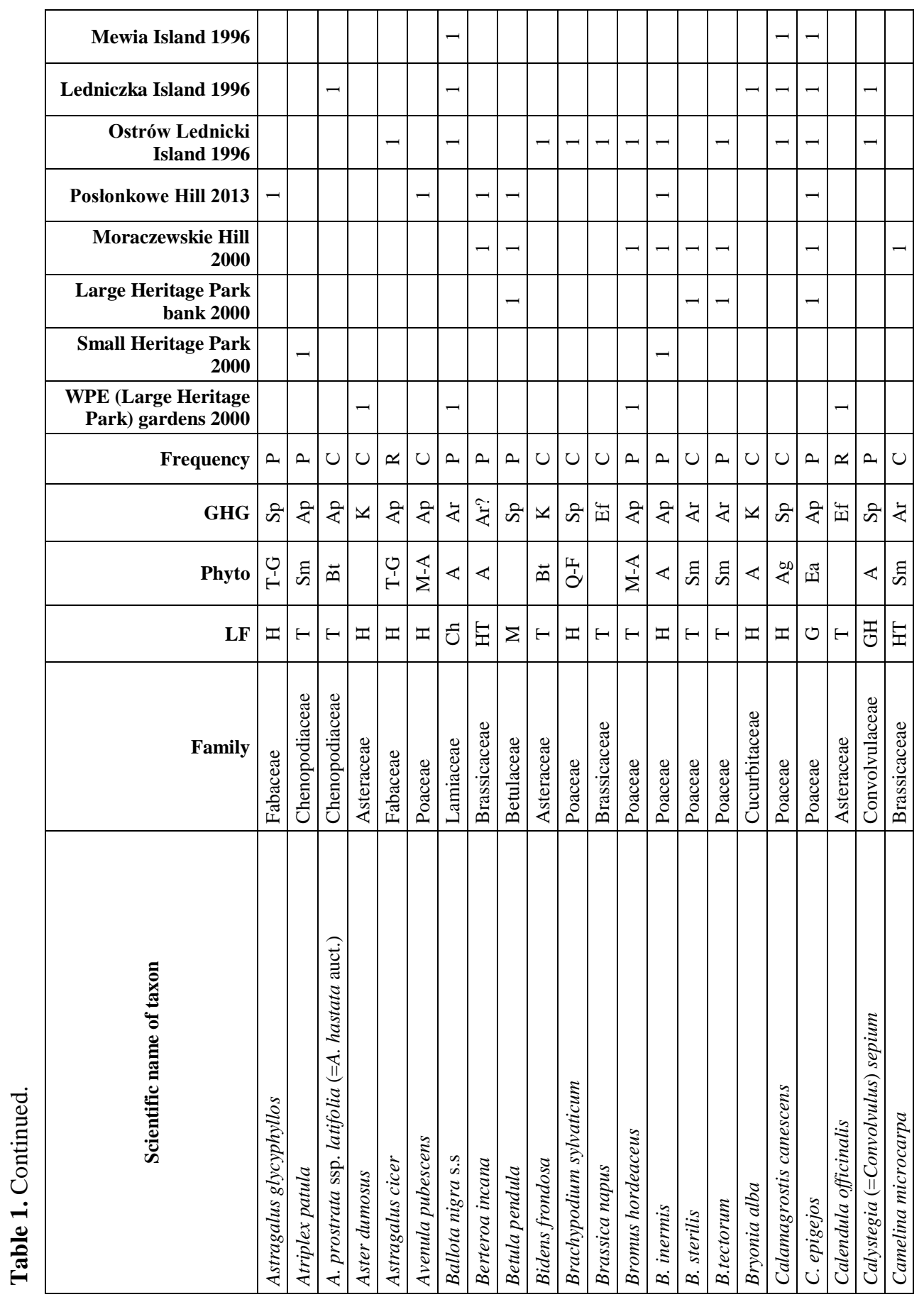




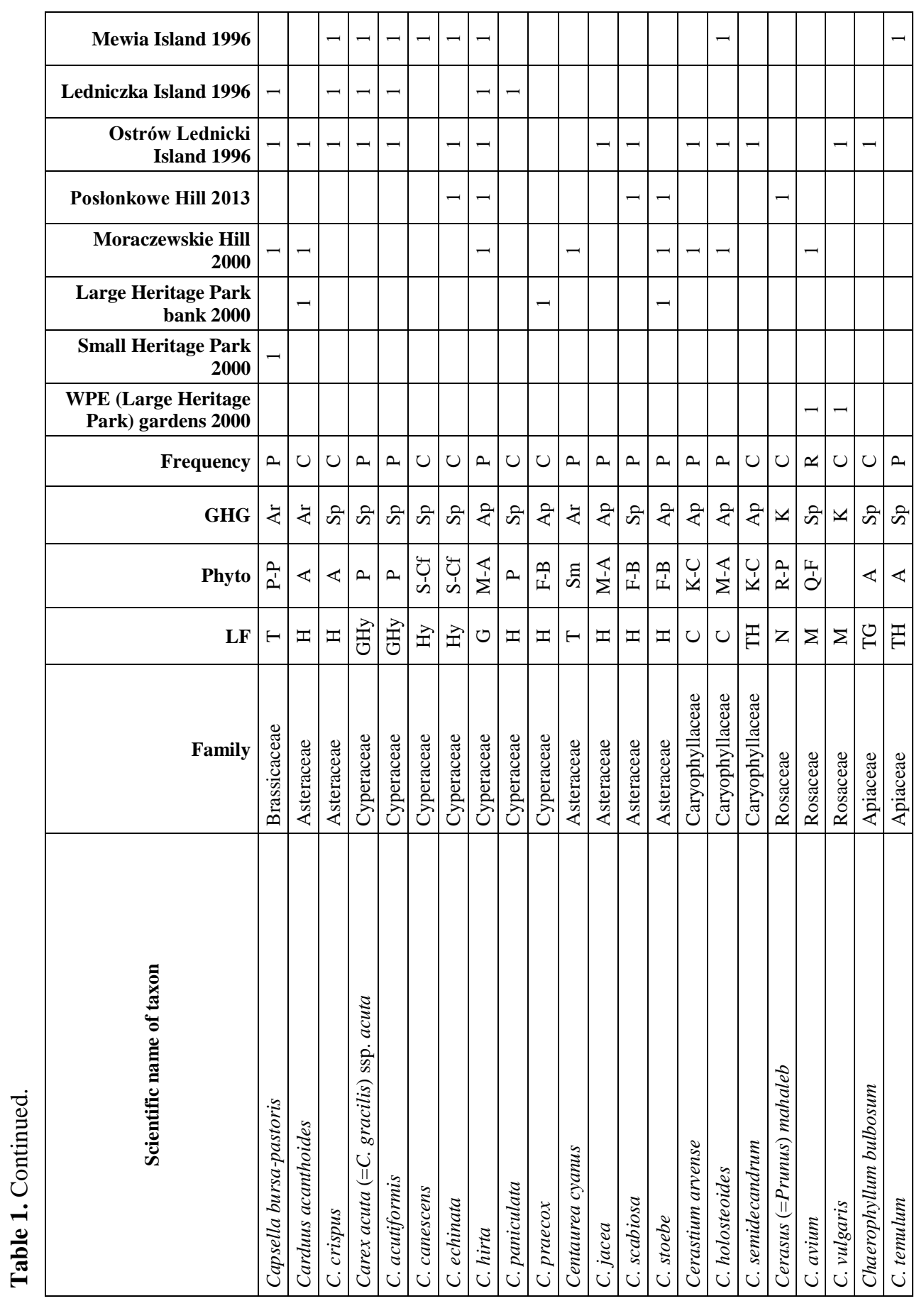




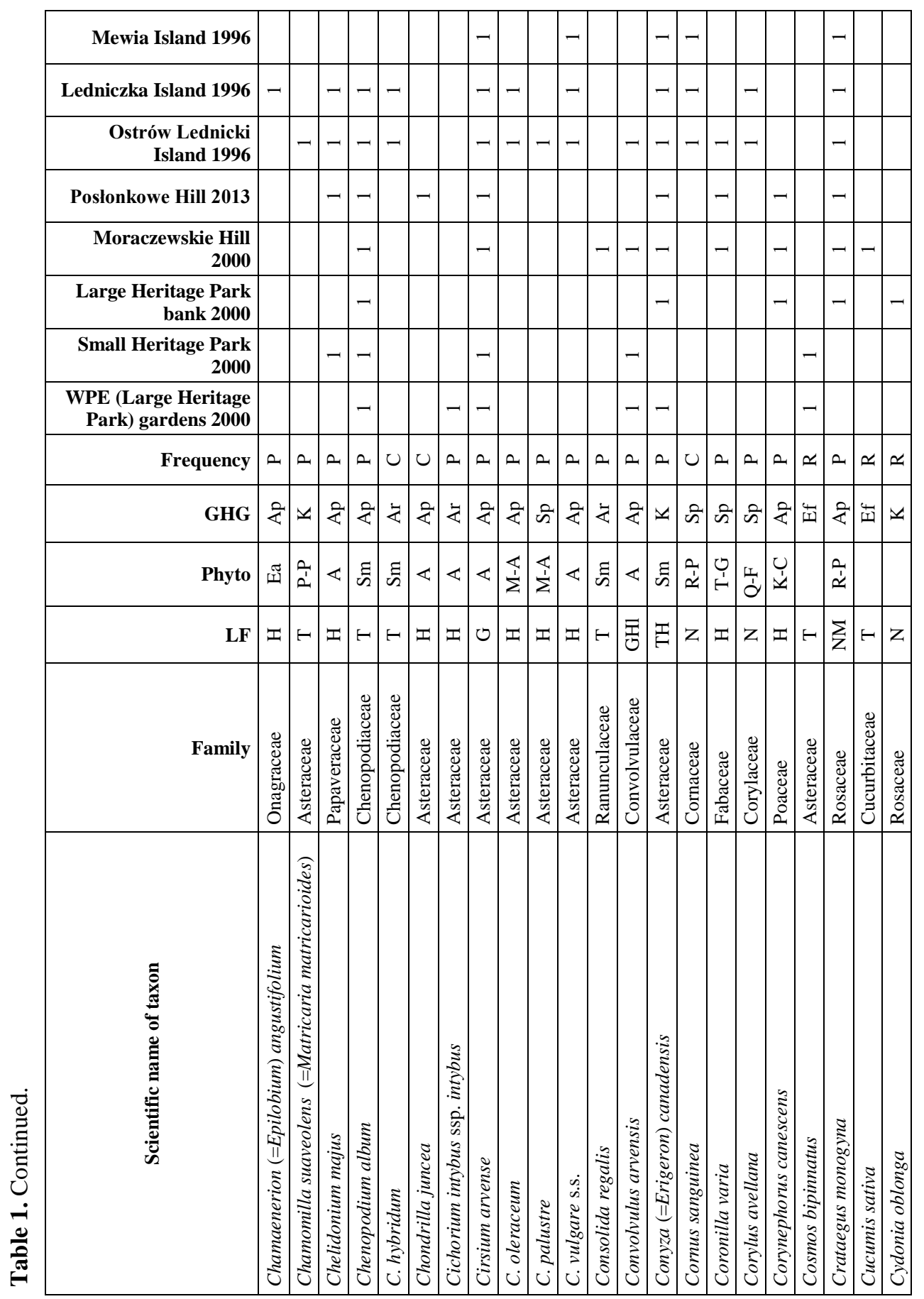




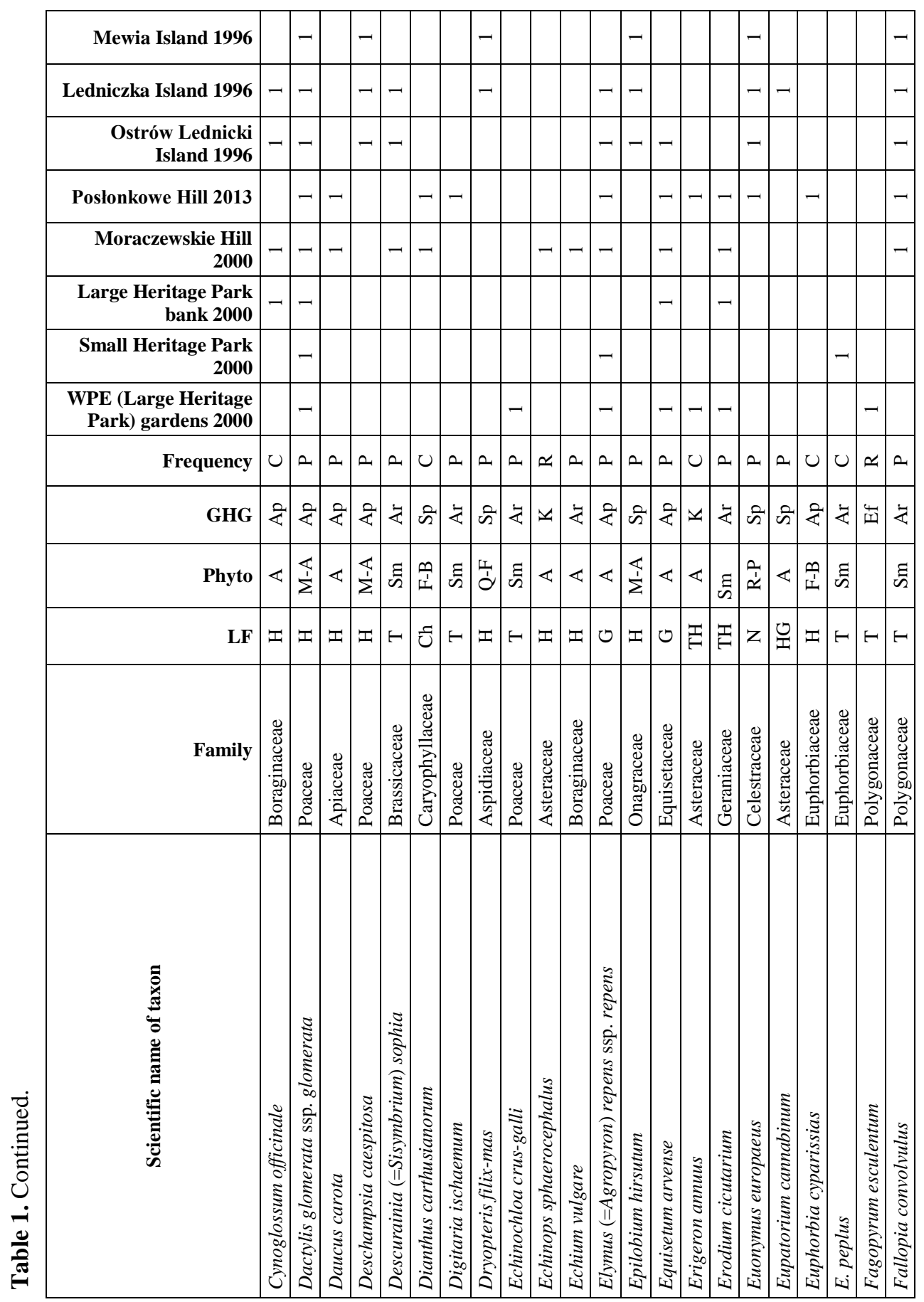




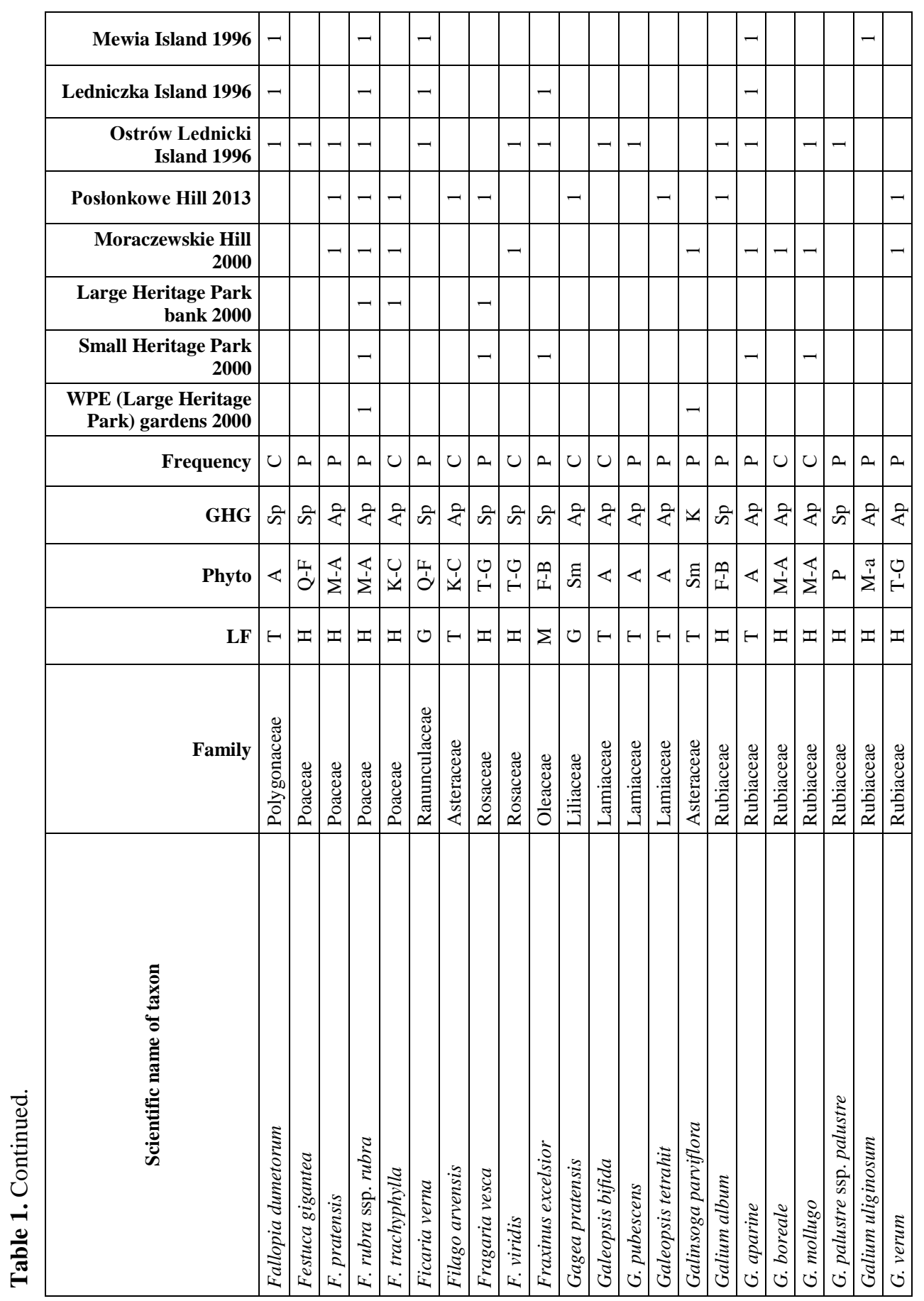




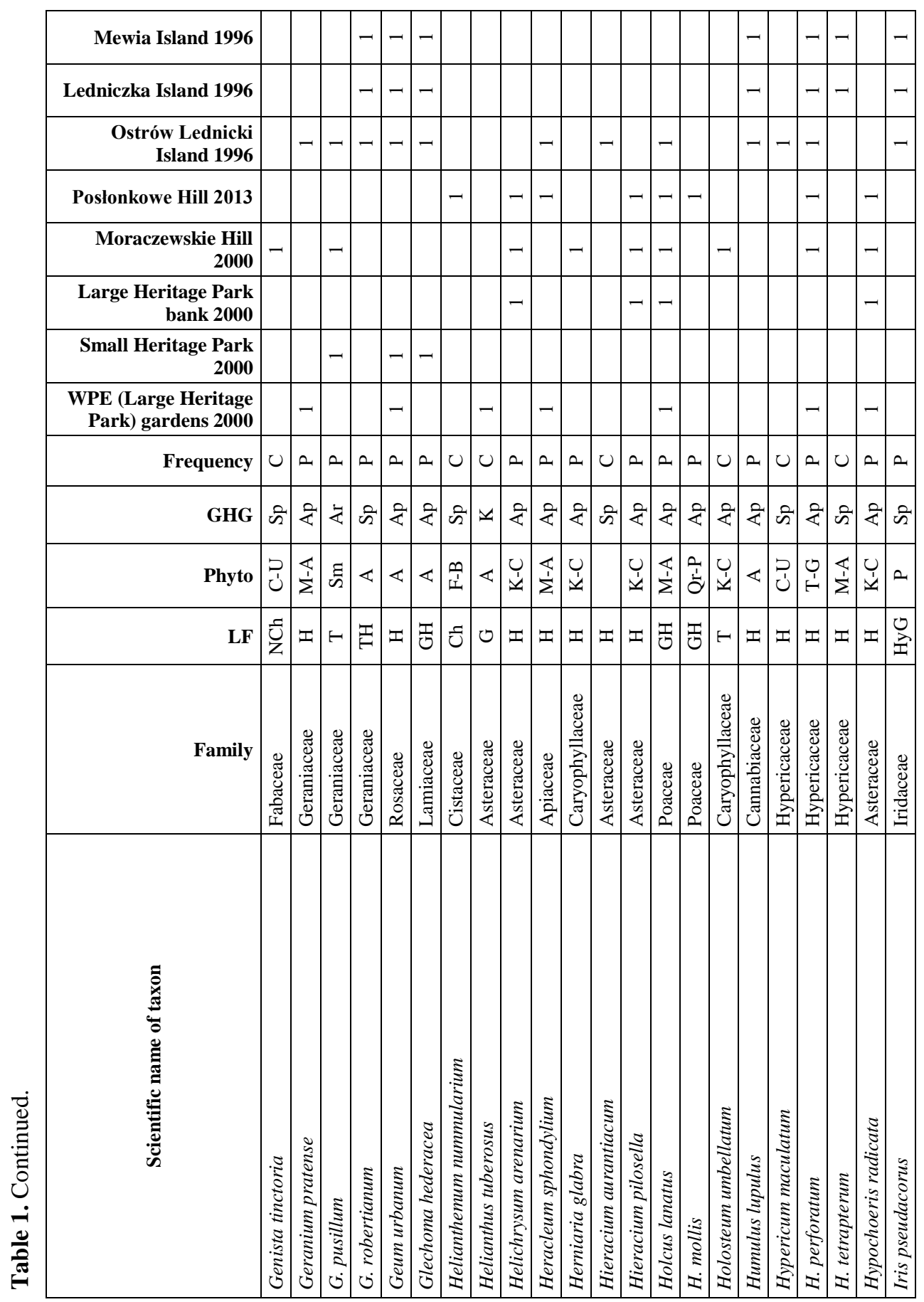




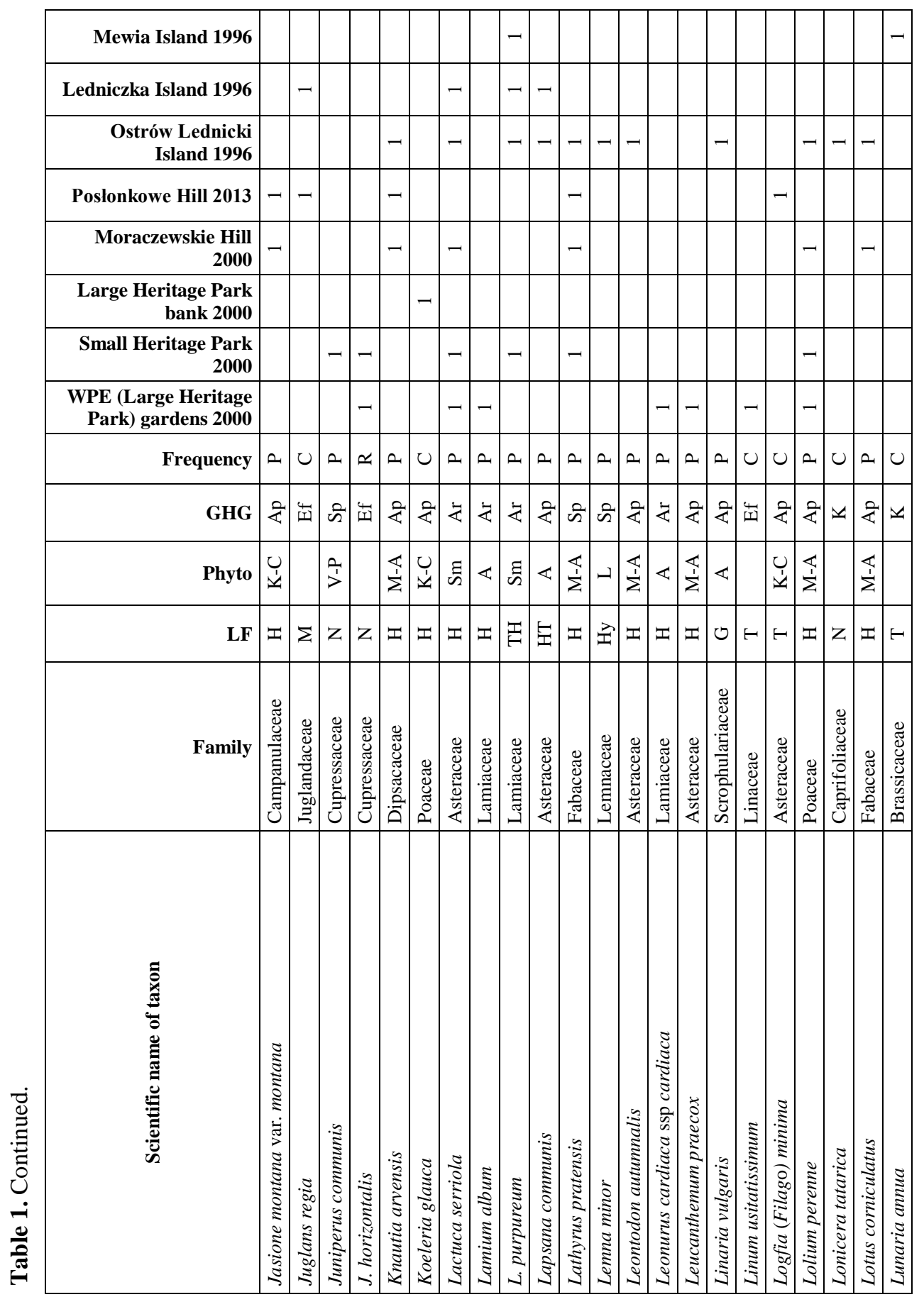




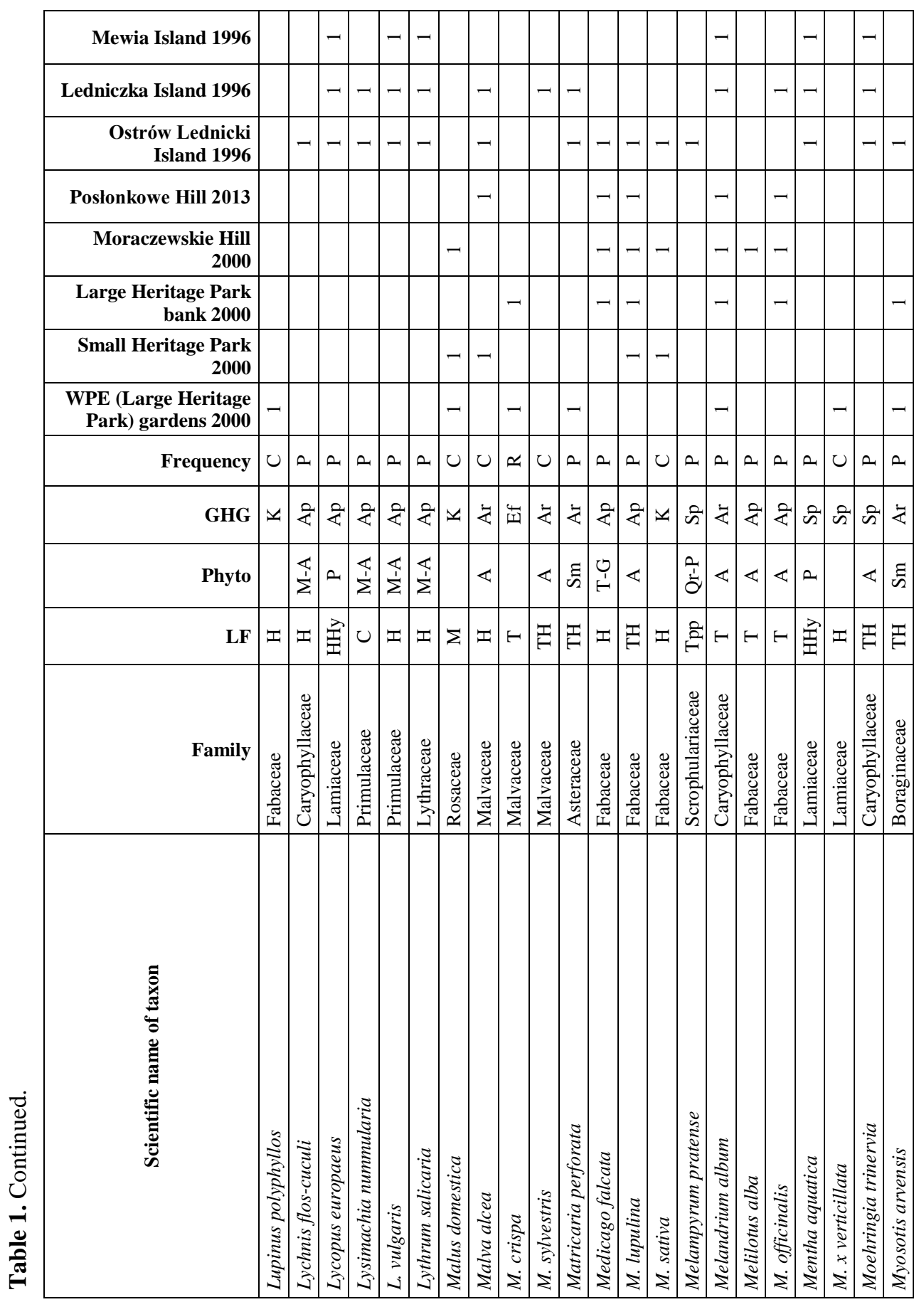




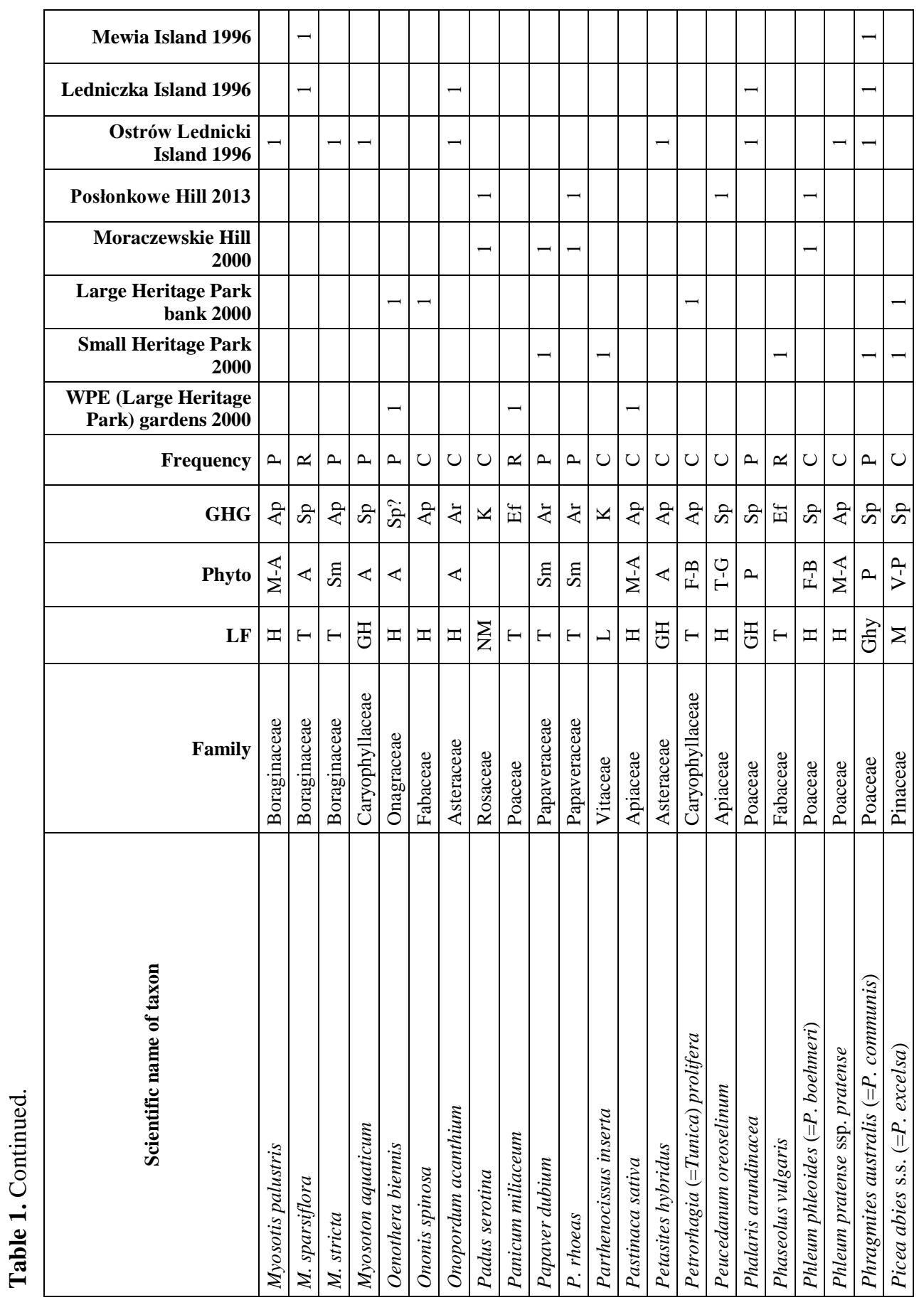




\begin{tabular}{|c|c|c|c|c|c|c|c|c|c|c|c|c|c|c|c|c|c|c|c|c|c|}
\hline Mewia Island 1996 & & & & - & & - & & & & & $-1-$ & $-1-$ & - & & & & & - & & & \\
\hline $\begin{array}{r}\text { Ledniczka Island } \\
1996\end{array}$ & & & & - & & -1 & -7 & - & & & - & - & - & & & & & & & & \\
\hline $\begin{array}{r}\text { Ostrów Lednicki } \\
\text { Island } 1996 \\
\end{array}$ & & - & & - & - & - & & -1 & - & & -7 & -1 & - & & - & - & - & & & - & - \\
\hline Posłonkowe Hill 2013 & - & & - & & - & & & - & & & & & & - & & & & & & & - \\
\hline $\begin{array}{r}\text { Moraczewskie Hill } \\
2000\end{array}$ & & - & -1 & & - & & & - & & - & & - & & & & & & & & - & - \\
\hline $\begin{array}{r}\text { Large Heritage Park } \\
\text { bank } 2000\end{array}$ & & - & - & & - & & & & & - & & - & & & & & & & & & \\
\hline $\begin{array}{r}\text { Small Heritage Park } \\
2000\end{array}$ & & & & & & - & & & - & & & - & & & & - & & & & & \\
\hline $\begin{array}{l}\text { WPE (Large Heritage } \\
\text { Park) gardens 2000 }\end{array}$ & & & & & - & - & & & - & & & & & & & & & & & & \\
\hline Frequency & 0 & 2 & $\infty$ & 0 & $\infty$ & 2 & 0 & $\infty$ & 2. & 2. & 2 & 4 & 2 & e & u & 2 & u & a & & 2 & 0 \\
\hline GHG & दे & की & के & 到 & 㝴 & से. & 워. & 这. & 这. & के & $n^{2}$ & a & 戠 & we & की & दे & के & की & ले & 安 & के \\
\hline Phyto & $\varangle$ & $\begin{array}{l}0 \\
\stackrel{1}{+} \\
\end{array}$ & $P^{2}$ & $z_{1}$ & $\stackrel{\mathbb{1}}{\Sigma}$ & $\pi_{i}^{\pi}$ & m & $\begin{array}{c}0 \\
\\
H\end{array}$ & in: & 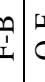 & $\frac{1}{2}$ & & $\sum^{4}$ & $\frac{1}{d}$ & & $\overbrace{}^{\prime}$ & & « & & $\mid \begin{array}{l}\mathbb{1} \\
\Sigma\end{array}$ & 龟 \\
\hline LF & \pm & \pm & $\Sigma$ & $\underline{\mathbf{I}}$ & \pm & \pm & \pm & \pm & $\mathbb{E}$ & $I=$ & $E=$ & 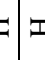 & \pm & 0 & 0 & $\mathrm{r}$ & $\Sigma$ & $\Sigma$ & & \pm & \pm \\
\hline Family & 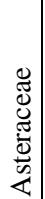 & 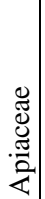 & 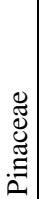 & 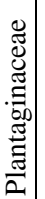 & 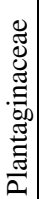 & 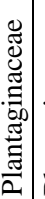 & 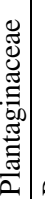 & 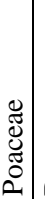 & 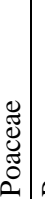 & & & & 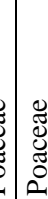 & 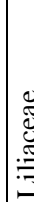 & 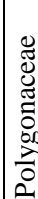 & & & 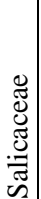 & & 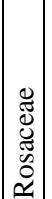 & 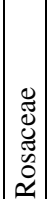 \\
\hline 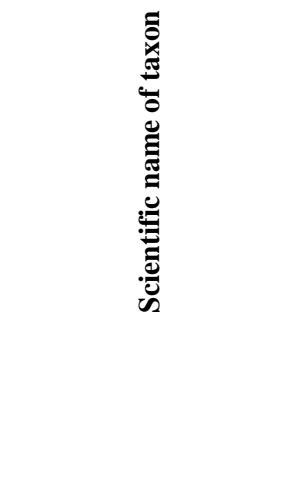 & 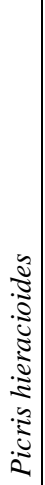 & 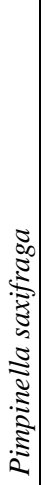 & 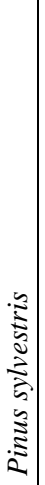 & 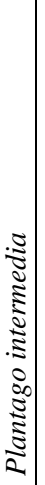 & 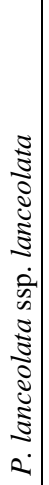 & 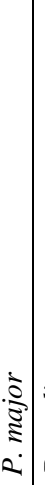 & : & 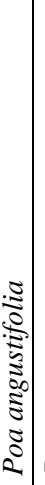 & 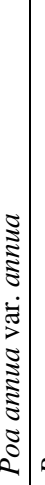 & $\begin{array}{c}0 \\
0 \\
0 \\
0\end{array}$ & 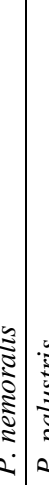 & 8 & 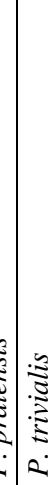 & 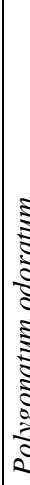 & 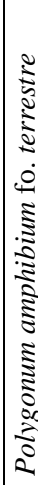 & 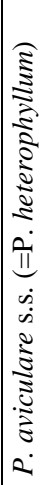 & 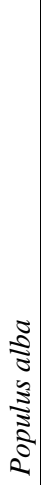 & & & 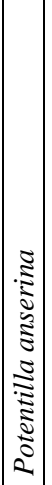 & 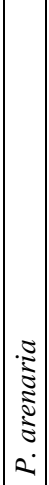 \\
\hline
\end{tabular}




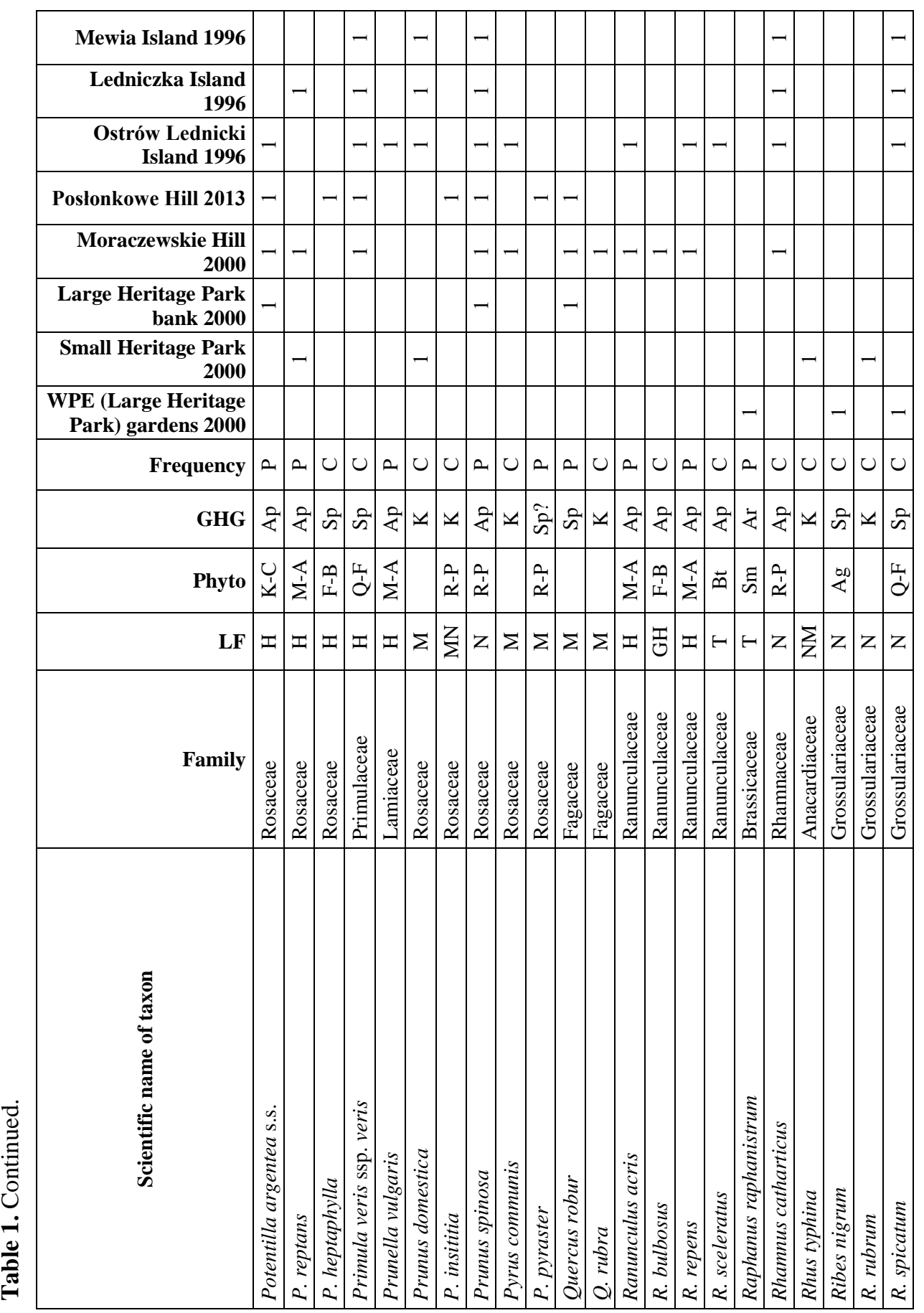




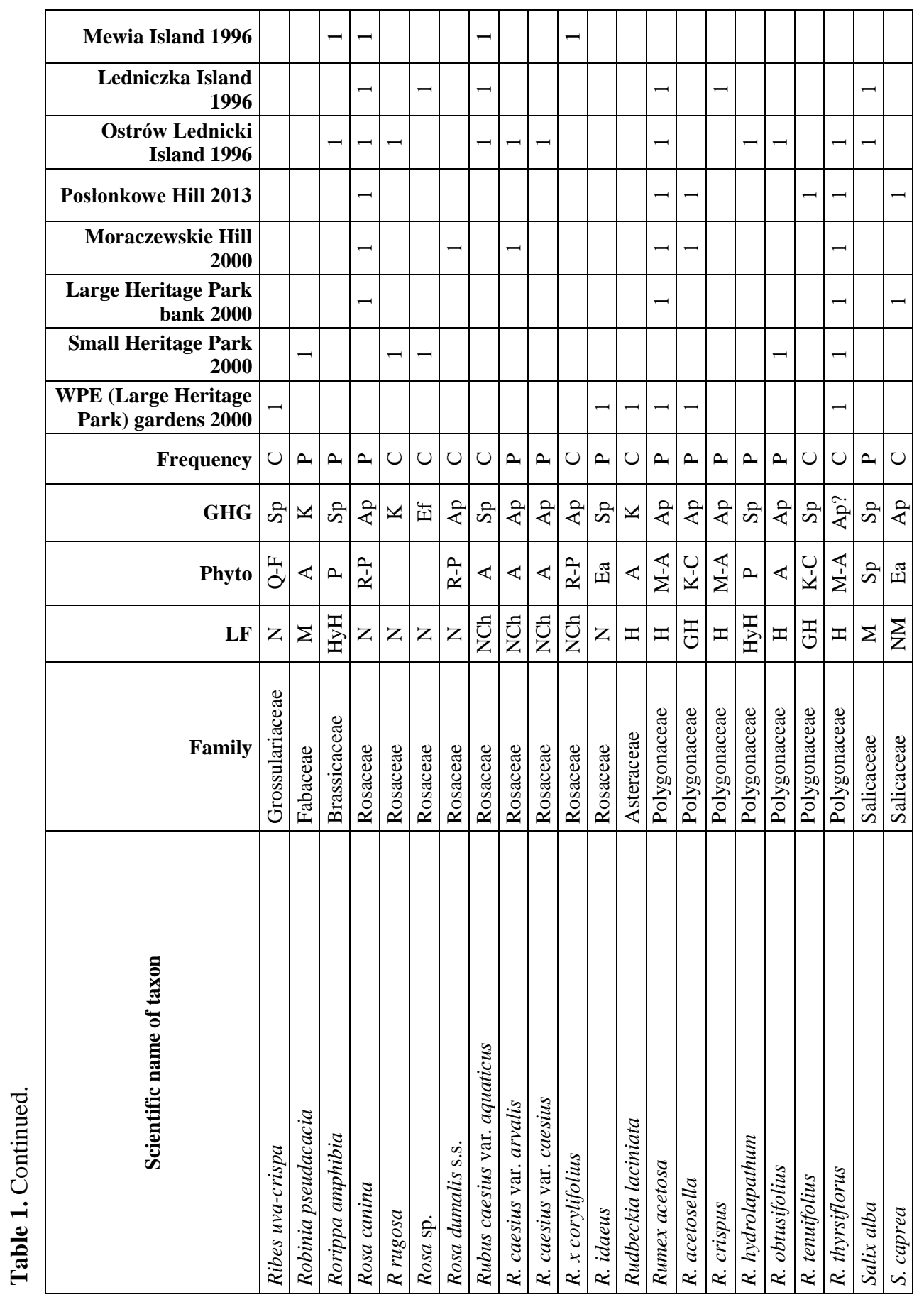




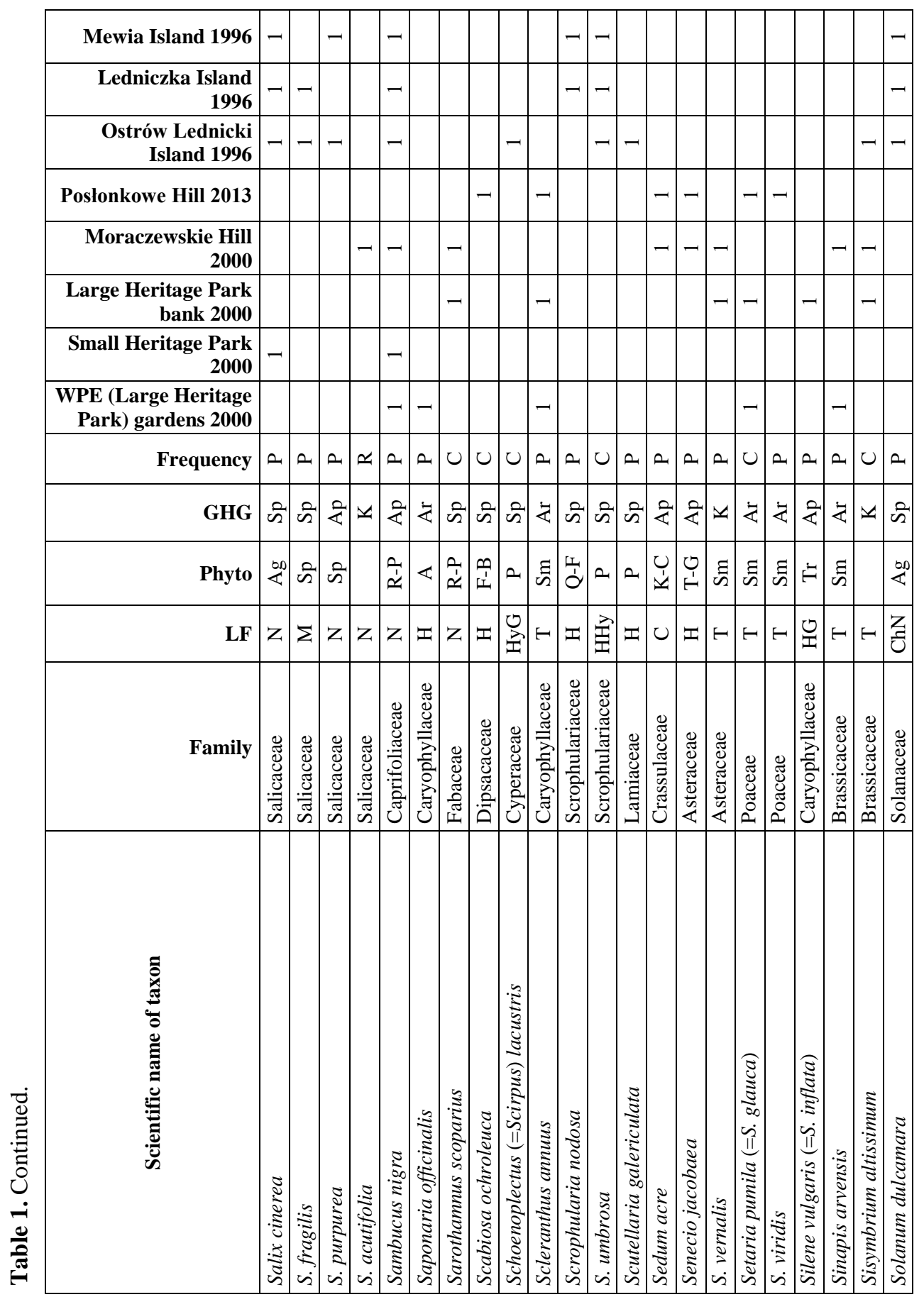




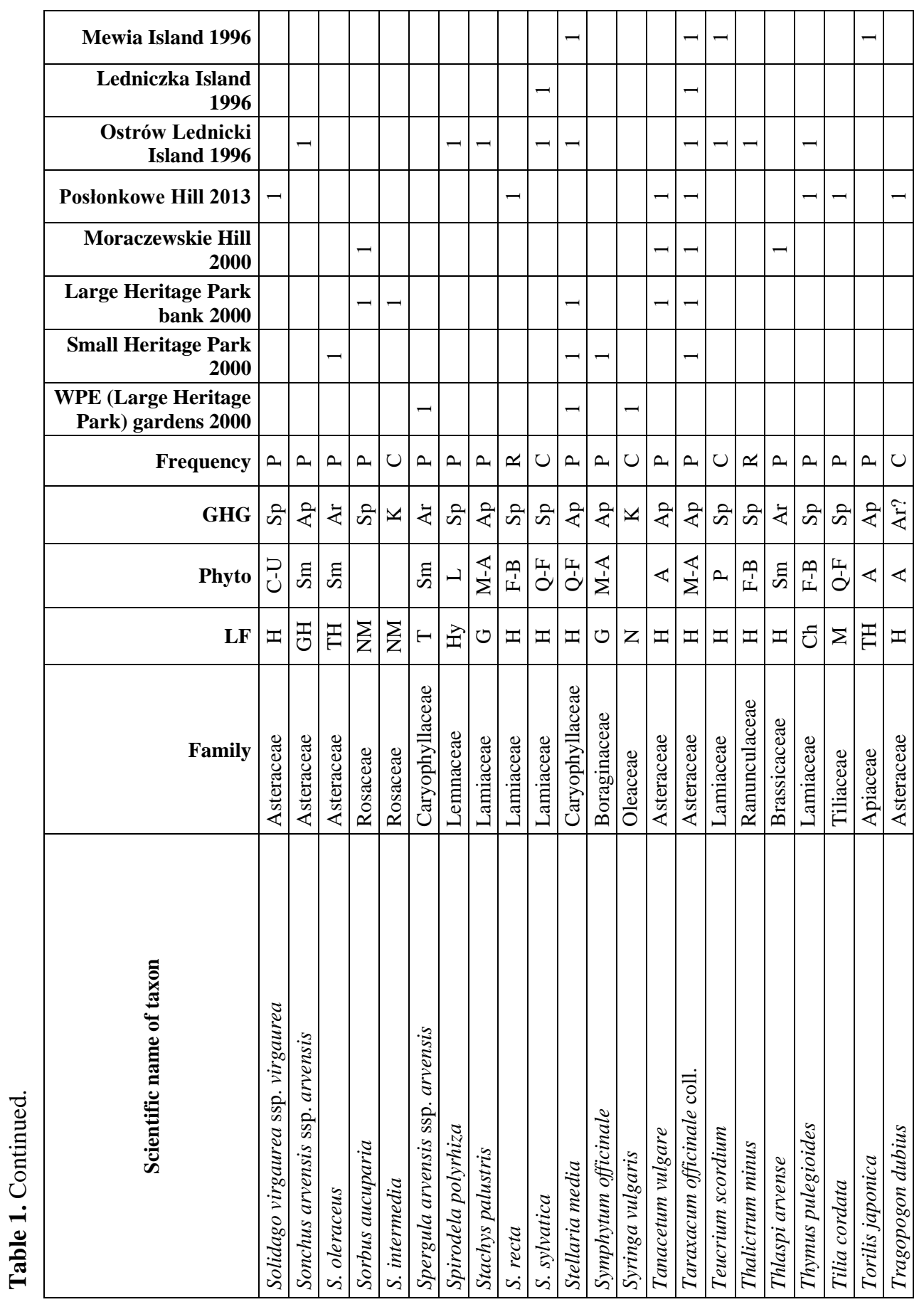




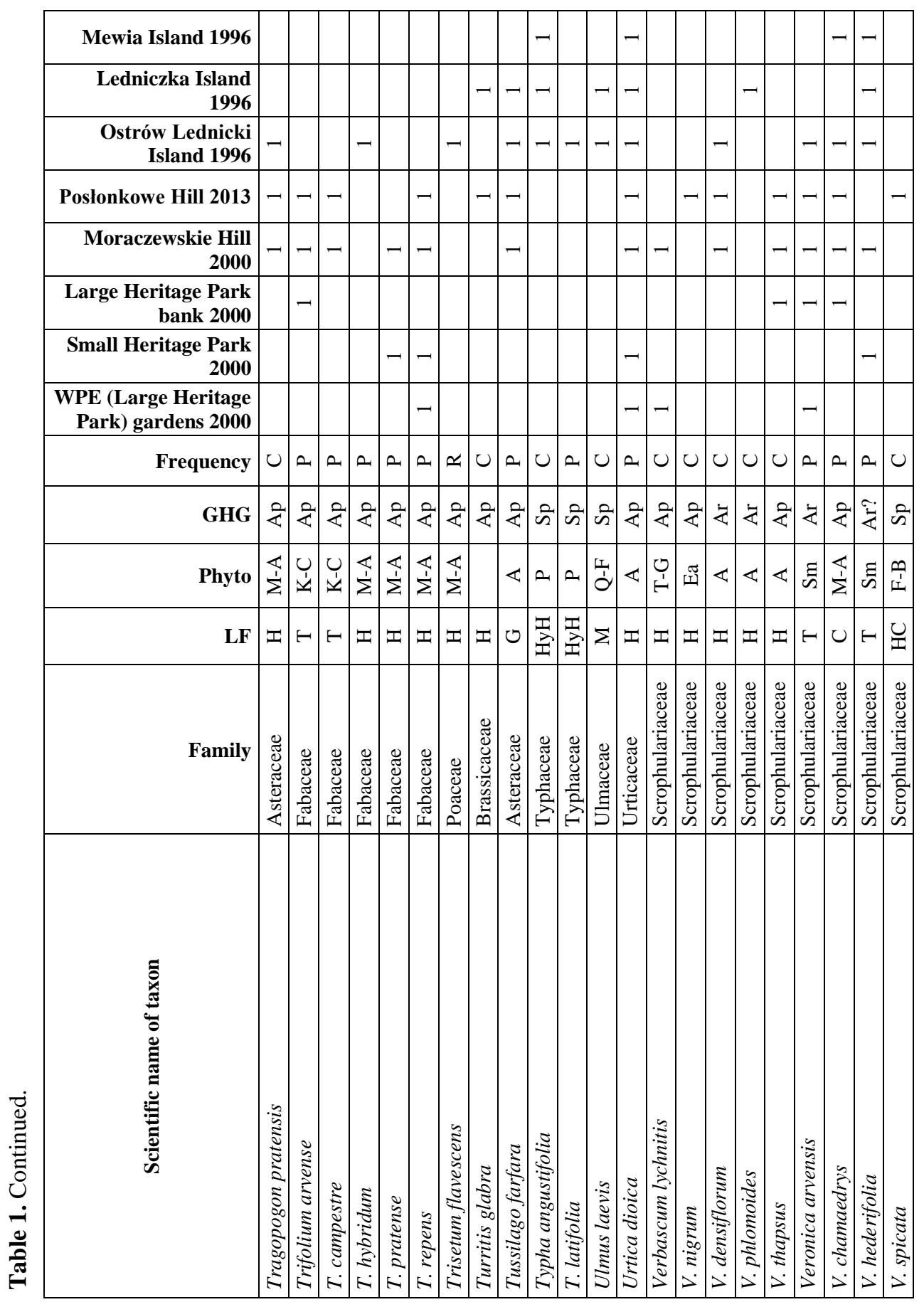




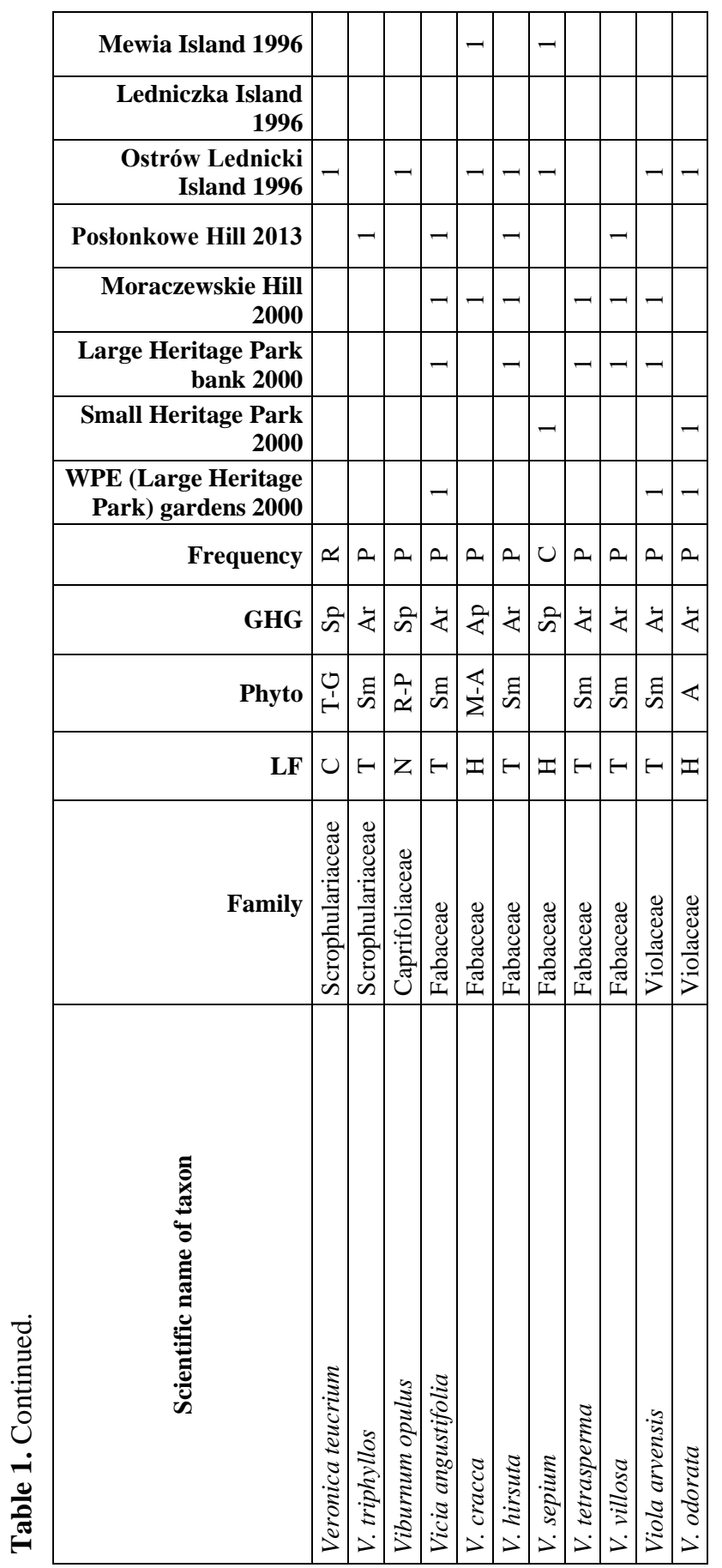




\begin{tabular}{|c|c|c|c|c|c|c|}
\hline 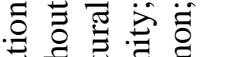 & Mewia Island 1996 & & กิ & & - & \\
\hline 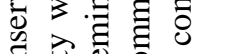 & Ledniczka Island 1996 & & กี & & ते & + \\
\hline ن & $\begin{array}{r}\text { Ostrów Lednicki } \\
\text { Island } 1996\end{array}$ & & तี & - & กี & \\
\hline 怘䓌 & Posłonkowe Hill 2013 & & & & & \\
\hline 忌芯 & $\begin{array}{r}\text { Moraczewskie Hill } \\
2000\end{array}$ & & & & & \\
\hline Z & $\begin{array}{r}\text { Large Heritage Park } \\
\text { bank } 2000\end{array}$ & & & & & \\
\hline d & $\begin{array}{r}\text { Small Heritage Park } \\
2000\end{array}$ & & & & & \\
\hline 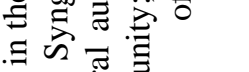 & $\begin{array}{r}\text { WPE (Large Heritage } \\
\text { Park) gardens } 2000\end{array}$ & 芯 & & & & \\
\hline 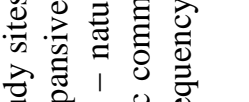 & DS. Minist & $\mid$ & & 畩 & 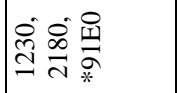 & 軠 \\
\hline 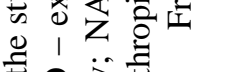 & $\begin{array}{r}\text { Frequency of } \\
\text { occurrence }\end{array}$ & 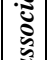 & 0 & $\simeq$ & u & u \\
\hline : 㭉芯 & Syngenesis & .ัँّ & 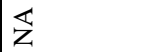 & 学 & $\mathrm{z}$ & z \\
\hline & Endangerment status & $\stackrel{\Sigma}{*}$ & 1 & $>$ & - & $>$ \\
\hline 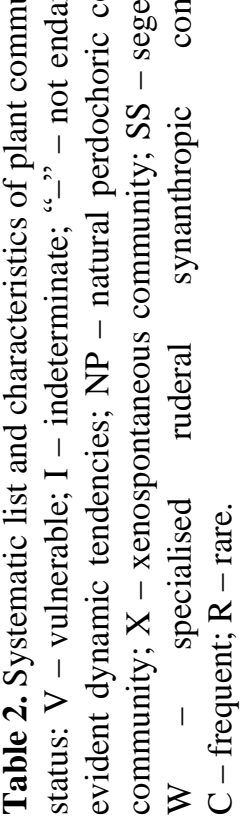 & 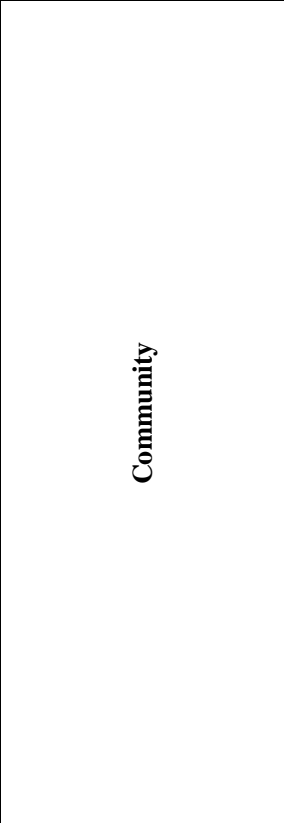 & 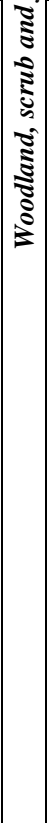 & 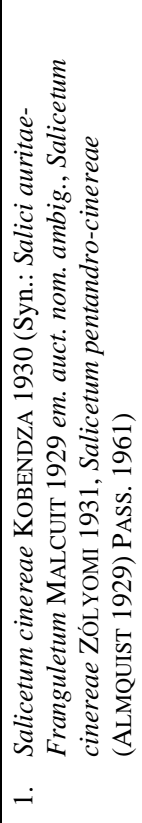 & 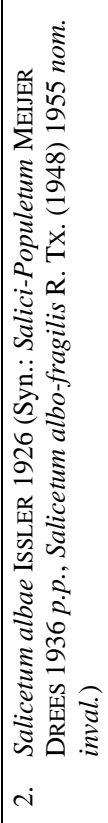 & 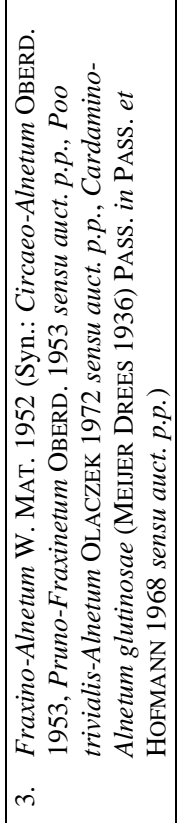 & 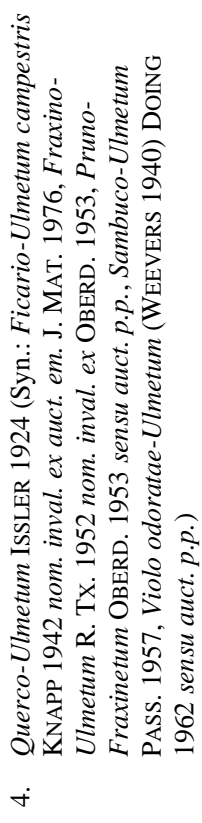 \\
\hline
\end{tabular}




\begin{tabular}{|c|c|c|c|c|c|c|c|c|}
\hline Mewia Island 1996 & + & & ลे & & & ลे & & \\
\hline Ledniczka Island 1996 & - & $m$ & & ते & & & & \\
\hline $\begin{array}{l}\text { Ostrów Lednicki } \\
\text { Island } 1996\end{array}$ & ปี & ते & บ็ & & & & & \\
\hline Poslonkowe Hill 2013 & สี & & & & & & & घี \\
\hline $\begin{array}{r}\text { Moraczewskie Hill } \\
2000\end{array}$ & & & & & + & & + & + \\
\hline $\begin{array}{r}\text { Large Heritage Park } \\
\text { bank } 2000\end{array}$ & & & & & & & - & + \\
\hline $\begin{array}{r}\text { Small Heritage Park } \\
2000\end{array}$ & & & & & & & & \\
\hline $\begin{array}{r}\text { WPE (Large Heritage } \\
\text { Park) gardens 2000 }\end{array}$ & & & & & & & & \\
\hline DS. Minist & & & & & & & & \\
\hline $\begin{array}{r}\text { Frequency of } \\
\text { occurrence }\end{array}$ & a & $a$ & $u$ & 0 & $\simeq$ & 0 & 0 & a \\
\hline Syngenesis & $\overleftrightarrow{z}$ & $\overleftrightarrow{z}$ & $\overleftrightarrow{z}$ & $\overleftrightarrow{z}$ & $\overleftrightarrow{z}$ & & $\overleftrightarrow{z}$ & $\overleftrightarrow{z}$ \\
\hline Endangerment status & 1 & 1 & - & - & $>$ & । & - & 1 \\
\hline ठ & 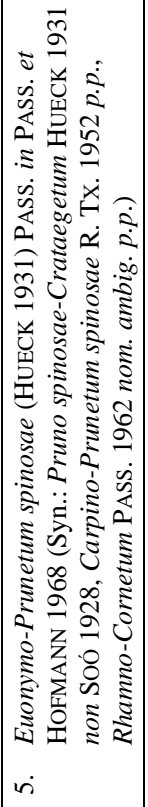 & 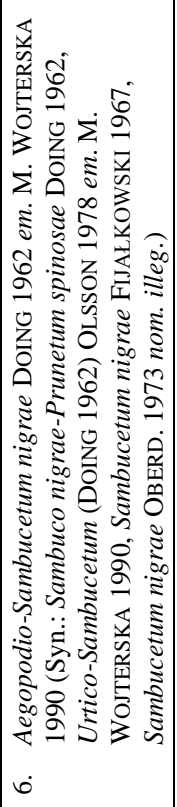 & 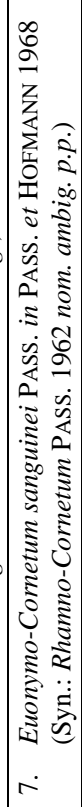 & 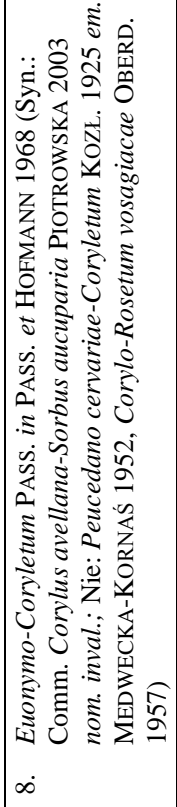 & 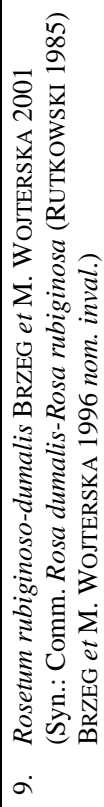 & 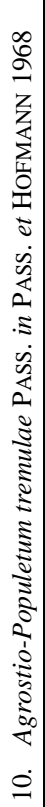 & 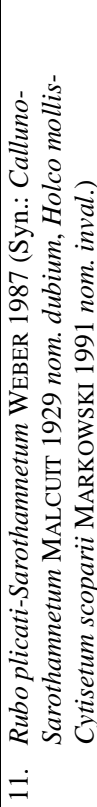 & 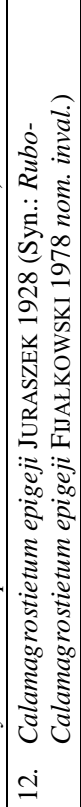 \\
\hline
\end{tabular}




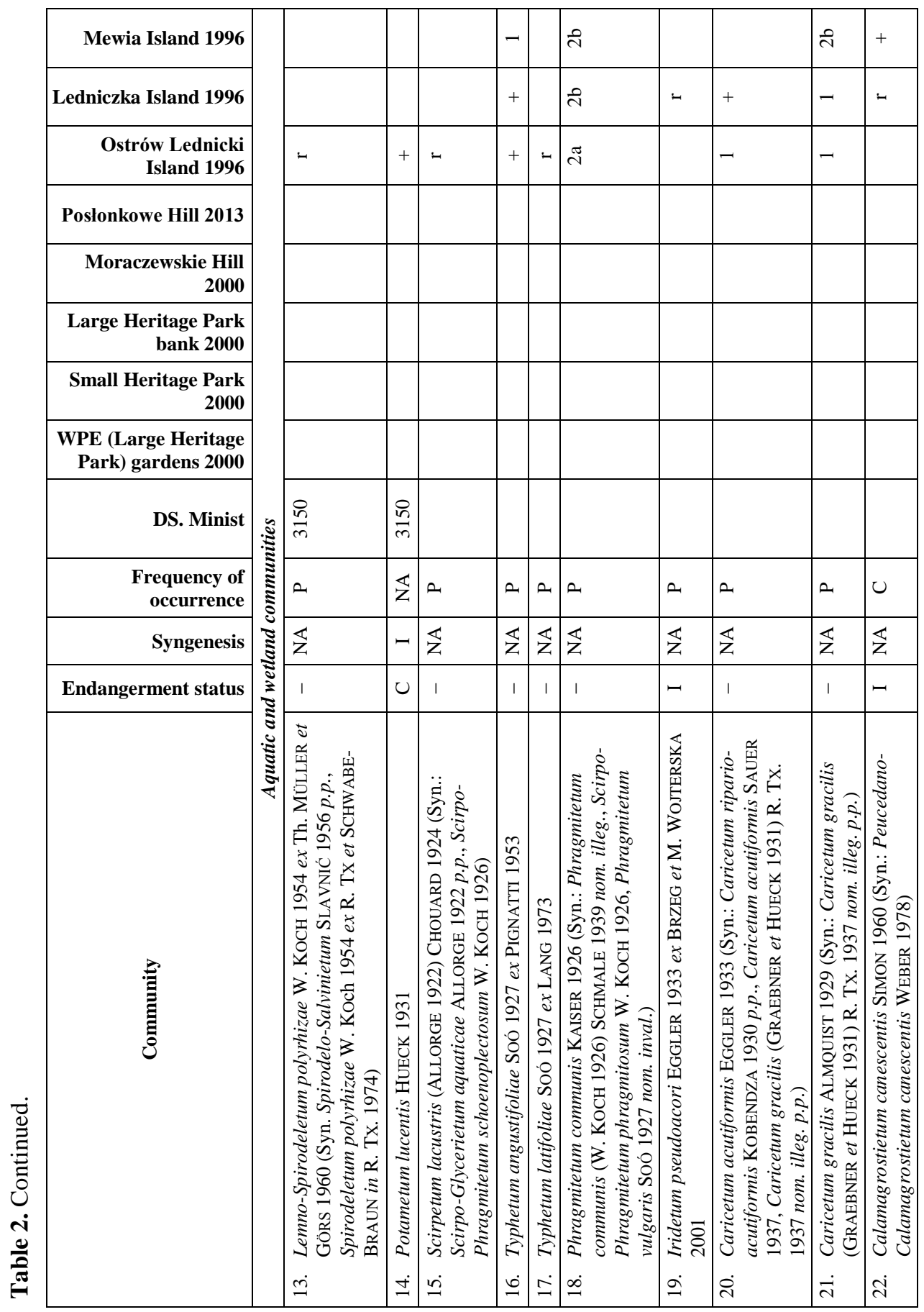




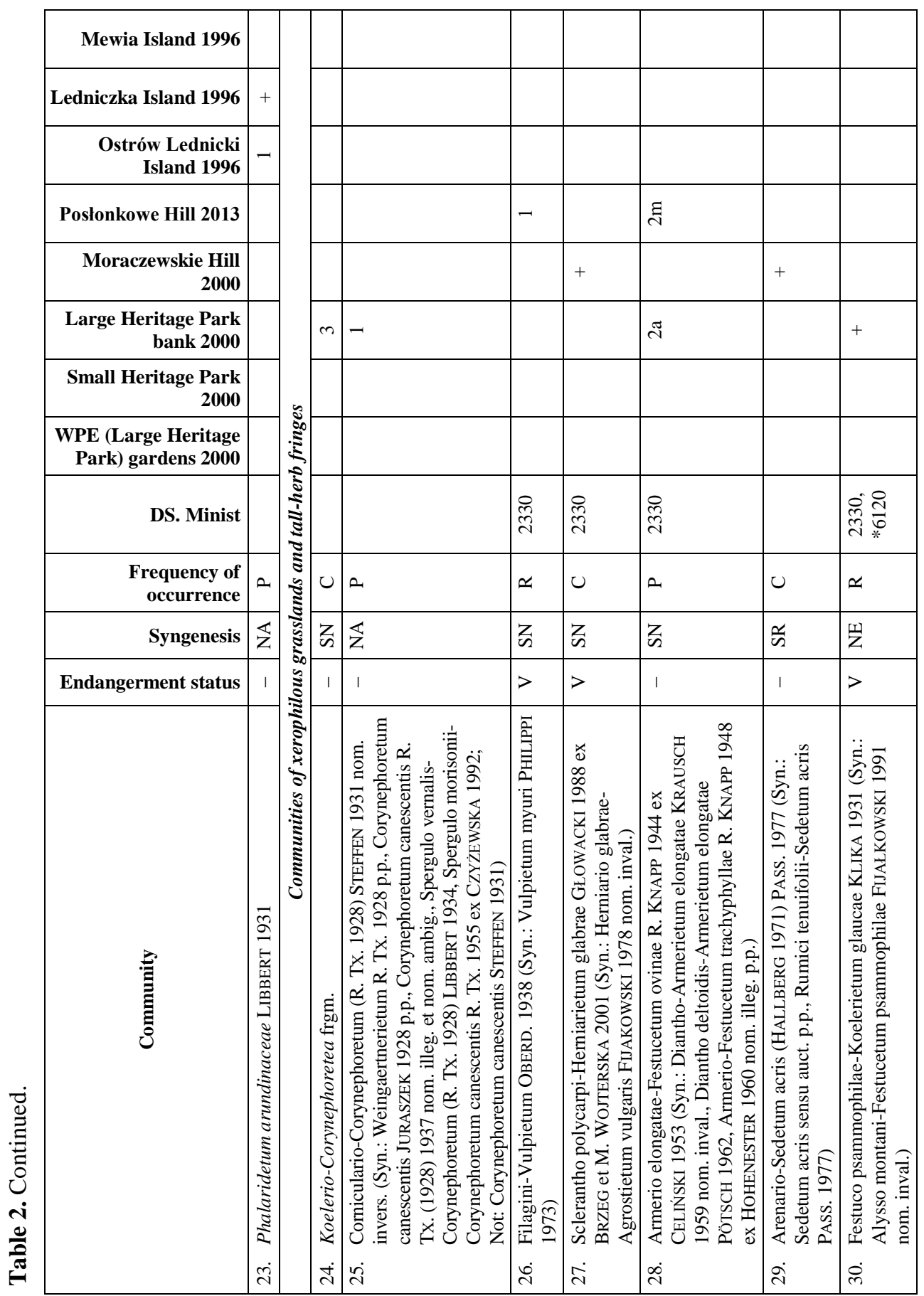




\begin{tabular}{|c|c|c|c|c|c|c|c|c|}
\hline Mewia Island 1996 & & & & & & & & \\
\hline Ledniczka Island 1996 & & & & & & & & \\
\hline $\begin{array}{r}\text { Ostrów Lednicki } \\
\text { Island } 1996\end{array}$ & & & & & - & - & + & $m$ \\
\hline Poslonkowe Hill 2013 & m & & ก็ & ते & & & & \\
\hline $\begin{array}{r}\text { Moraczewskie Hill } \\
2000\end{array}$ & + & + & & & & & & ते \\
\hline $\begin{array}{r}\text { Large Heritage Park } \\
\text { bank } 2000\end{array}$ & & & & & & & & - \\
\hline $\begin{array}{r}\text { Small Heritage Park } \\
2000\end{array}$ & & & & & & & & - \\
\hline $\begin{array}{l}\text { WPE (Large Heritage } \\
\text { Park) gardens } 2000\end{array}$ & & & & & & & & - \\
\hline DS. Minist & & $\stackrel{ }{\text { तु }}$ & & & & & & $\frac{0}{30}$ \\
\hline $\begin{array}{r}\text { Frequency of } \\
\text { occurrence }\end{array}$ & u & $\simeq$ & a & 0 & $\bar{\Xi}$ & 0 & 0 & 2 \\
\hline Syngenesis & $z$ & $\overleftrightarrow{z}$ & $\overleftrightarrow{z}$ & $\overleftrightarrow{z}$ & $\overleftrightarrow{\text { Z }}$ & z & z & z \\
\hline Endangerment status & 1 & $>$ & 1 & 1 & 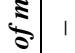 & I & $>$ & $>$ \\
\hline ن & 童 & 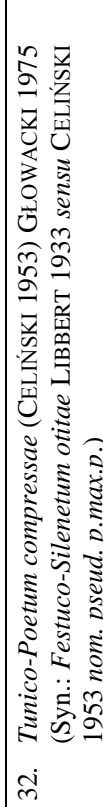 & 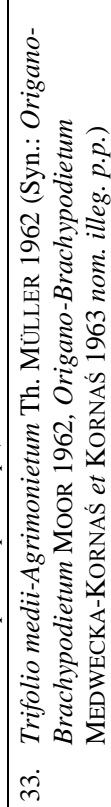 & 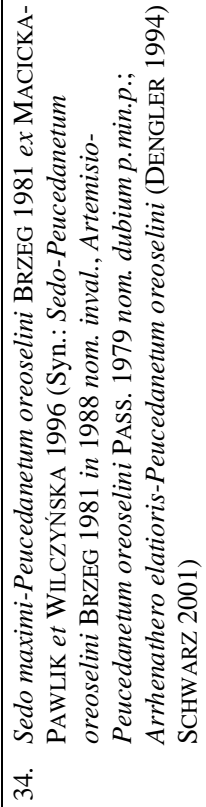 & 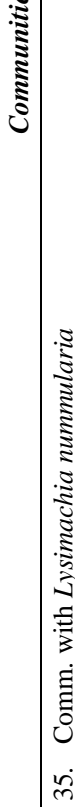 & 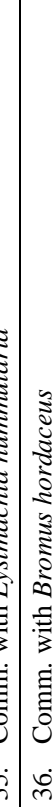 & 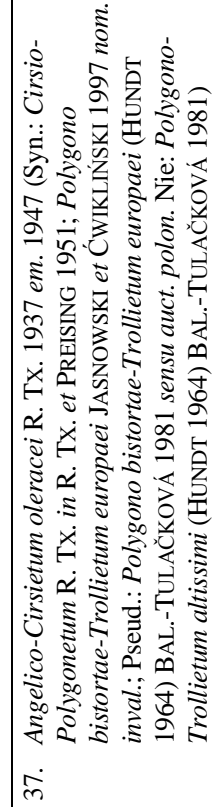 & 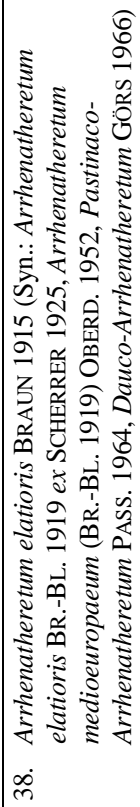 \\
\hline
\end{tabular}




\begin{tabular}{|c|c|c|c|c|c|c|}
\hline Mewia Island 1996 & & & & & สี & \\
\hline Ledniczka Island 1996 & & & & & บ็ & \\
\hline $\begin{array}{r}\text { Ostrów Lednicki } \\
\text { Island } 1996\end{array}$ & ปี & - & + & - & $m$ & \\
\hline Poslonkowe Hill 2013 & & & & & $\simeq$ & \\
\hline $\begin{array}{r}\text { Moraczewskie Hill } \\
2000\end{array}$ & & & & & + & \\
\hline $\begin{array}{r}\text { Large Heritage Park } \\
\text { bank } 2000\end{array}$ & & & & & & \\
\hline $\begin{array}{r}\text { Small Heritage Park } \\
2000\end{array}$ & in & - & & & + & \\
\hline $\begin{array}{l}\text { WPE (Large Heritage } \\
\text { Park) gardens } 2000\end{array}$ & & ก็ & & & & + \\
\hline DS. Minist & & & & & & \\
\hline $\begin{array}{r}\text { Frequency of } \\
\text { occurrence }\end{array}$ & u & a & u & $a$ & $a$ & u \\
\hline Syngenesis & 台 & Z & z & Z & $\mathbb{z}$ & $x$ \\
\hline Endangerment status & - & 1 & 1 & 1 & 1 & 0 \\
\hline ठ & 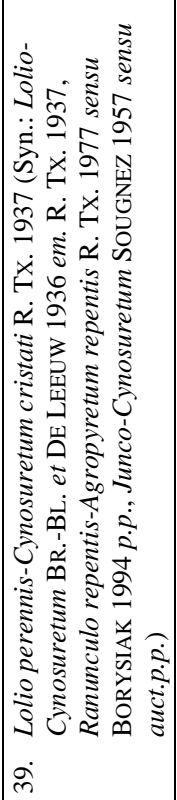 & 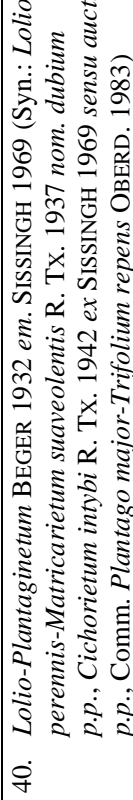 & 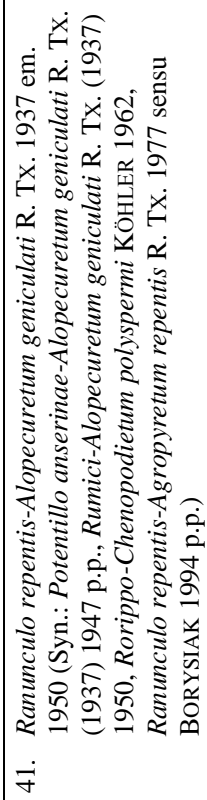 & 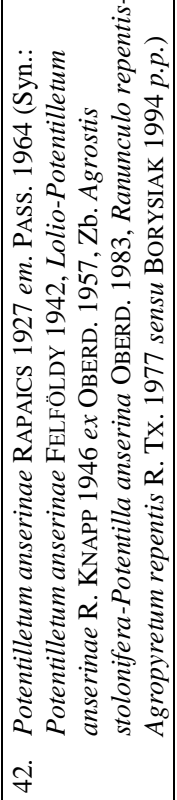 & 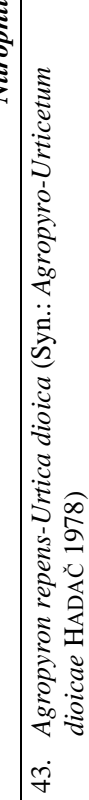 & 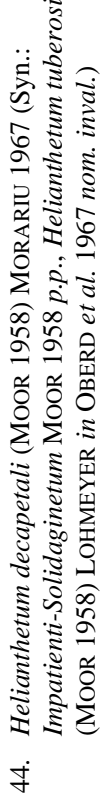 \\
\hline
\end{tabular}




\begin{tabular}{|c|c|c|c|c|c|c|c|c|c|c|c|c|}
\hline Mewia Island 1996 & & & & & สี & & & & & + & & \\
\hline \multicolumn{13}{|l|}{ Ledniczka Island 1996} \\
\hline $\begin{array}{r}\text { Ostrów Lednicki } \\
\text { Island } 1996\end{array}$ & - & तี & - & + & तิ & + & ते & + & + & & & + \\
\hline \multicolumn{13}{|l|}{ Posłonkowe Hill 2013} \\
\hline $\begin{array}{r}\text { Moraczewskie Hill } \\
2000\end{array}$ & & & & + & & & & & + & & & \\
\hline \multicolumn{13}{|l|}{$\begin{array}{r}\text { Large Heritage Park } \\
\text { bank } 2000 \\
\end{array}$} \\
\hline $\begin{array}{r}\text { Small Heritage Park } \\
2000\end{array}$ & & & & & + & & & + & + & & - & \\
\hline $\begin{array}{r}\text { WPE (Large Heritage } \\
\text { Park) gardens } 2000\end{array}$ & & & & + & & & & & & & & \\
\hline DS. Minist & 尽 & 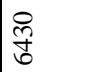 & 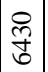 & 官 & 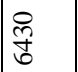 & & ণ্ণি & 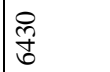 & & 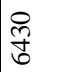 & & î \\
\hline $\begin{array}{r}\text { Frequency of } \\
\text { occurrence }\end{array}$ & a & $U$ & $U$ & $a$ & 2 & $u$ & U & $u$ & $U$ & $u$ & $u$ & $u$ \\
\hline Syngenesis & $\mathbb{z}$ & $\overleftrightarrow{z}$ & $\overleftrightarrow{z}$ & $\overleftrightarrow{z}$ & $\overleftrightarrow{z}$ & $\overleftrightarrow{z}$ & $\mathbb{z}$ & $\overleftrightarrow{Z}$ & $\mathbb{z}$ & $\overleftrightarrow{z}$ & $\overleftrightarrow{z}$ & $\overleftrightarrow{z}$ \\
\hline Endangerment status & 1 & 1 & 1 & 1 & 1 & 1 & - & 1 & । & 1 & । & । \\
\hline 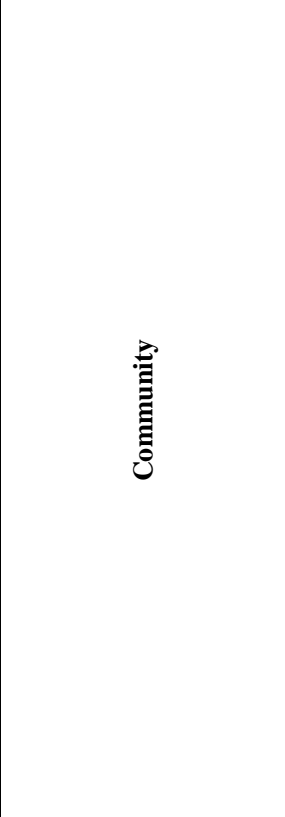 & 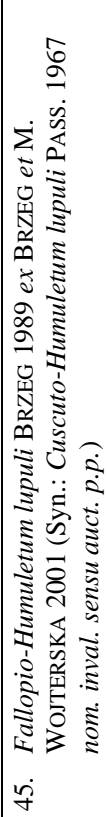 & 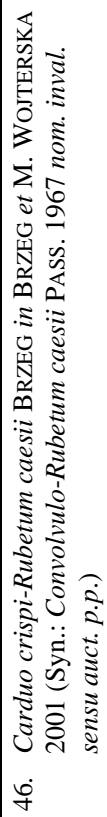 & 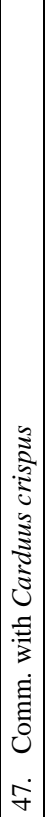 & 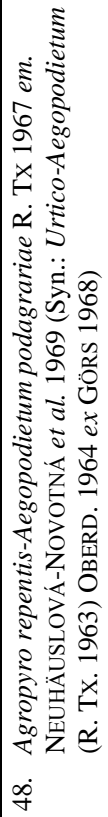 & 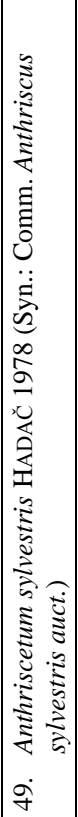 & 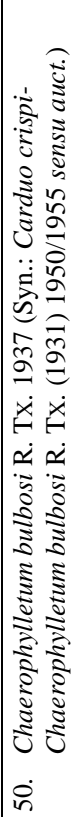 & 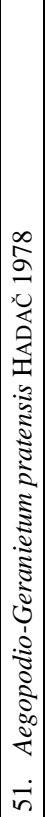 & 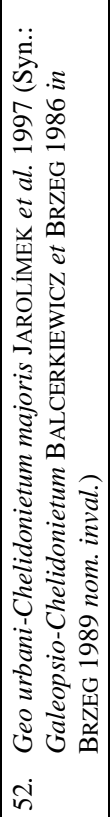 & 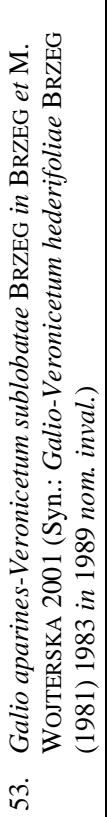 & 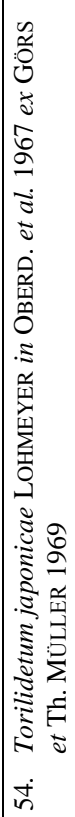 & 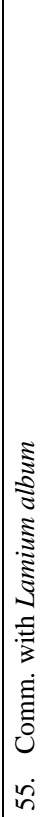 & 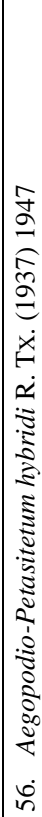 \\
\hline
\end{tabular}




\begin{tabular}{|c|c|c|c|c|c|c|c|c|c|c|}
\hline Mewia Island 1996 & & & & & & & & & + & \\
\hline Ledniczka Island 1996 & & & & & & & & & + & \\
\hline $\begin{array}{r}\text { Ostrów Lednicki } \\
\text { Island } 1996\end{array}$ & & - & & & & + & $m$ & & + & \\
\hline Posłonkowe Hill 2013 & + & & & & घี & & & & & \\
\hline $\begin{array}{r}\text { Moraczewskie Hill } \\
2000\end{array}$ & & & + & + & ก็ & & $\vec{\lambda}$ & + & & + \\
\hline $\begin{array}{r}\text { Large Heritage Park } \\
\text { bank } 2000\end{array}$ & & & - & & & & & & & \\
\hline $\begin{array}{r}\text { Small Heritage Park } \\
2000\end{array}$ & & & & & & & & & & \\
\hline $\begin{array}{l}\text { WPE (Large Heritage } \\
\text { Park) gardens } 2000\end{array}$ & & & & & ก็ & & & & + & \\
\hline DS. Minist & & & & & & & & & & \\
\hline $\begin{array}{r}\text { Frequency of } \\
\text { occurrence }\end{array}$ & u & 0 & 2 & $\simeq$ & a & 0 & 0 & 2 & a & u \\
\hline Syngenesis & $\ddot{\varkappa}$ & 丞 & 乩 & $\tilde{w}$ & $\tilde{\omega}$ & $\approx$ & $\tilde{\omega}$ & $\frac{\tilde{\omega}}{\omega}$ & $\ddot{\omega}$ & $z_{s}$ \\
\hline Endangerment status & 1 & 1 & 1 & - & 1 & 0 & 0 & 1 & 1 & \\
\hline 㞯 & 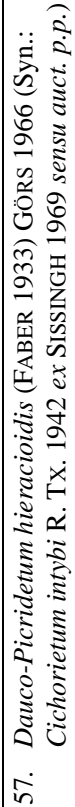 & 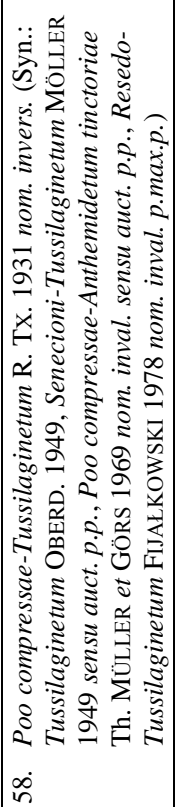 & 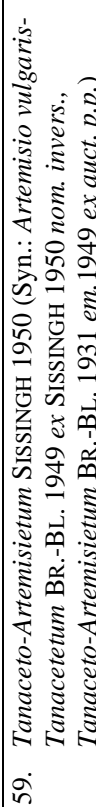 & 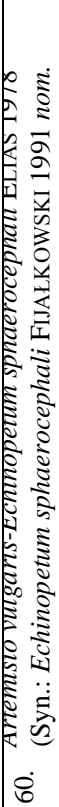 & 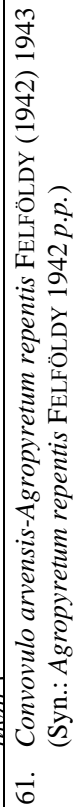 & 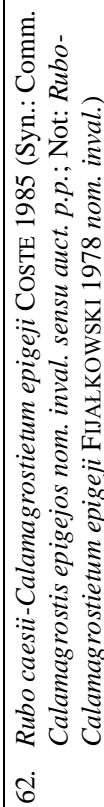 & 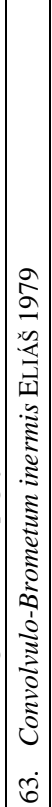 & 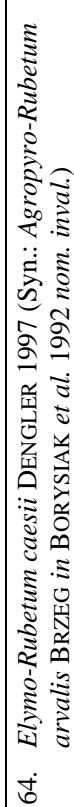 & 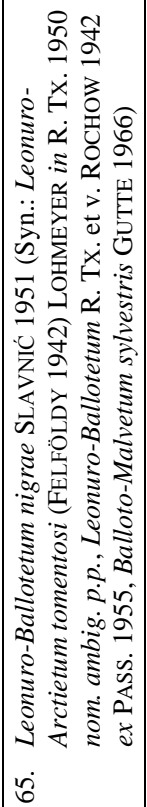 & 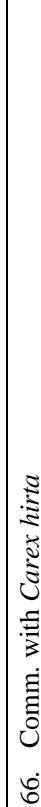 \\
\hline
\end{tabular}




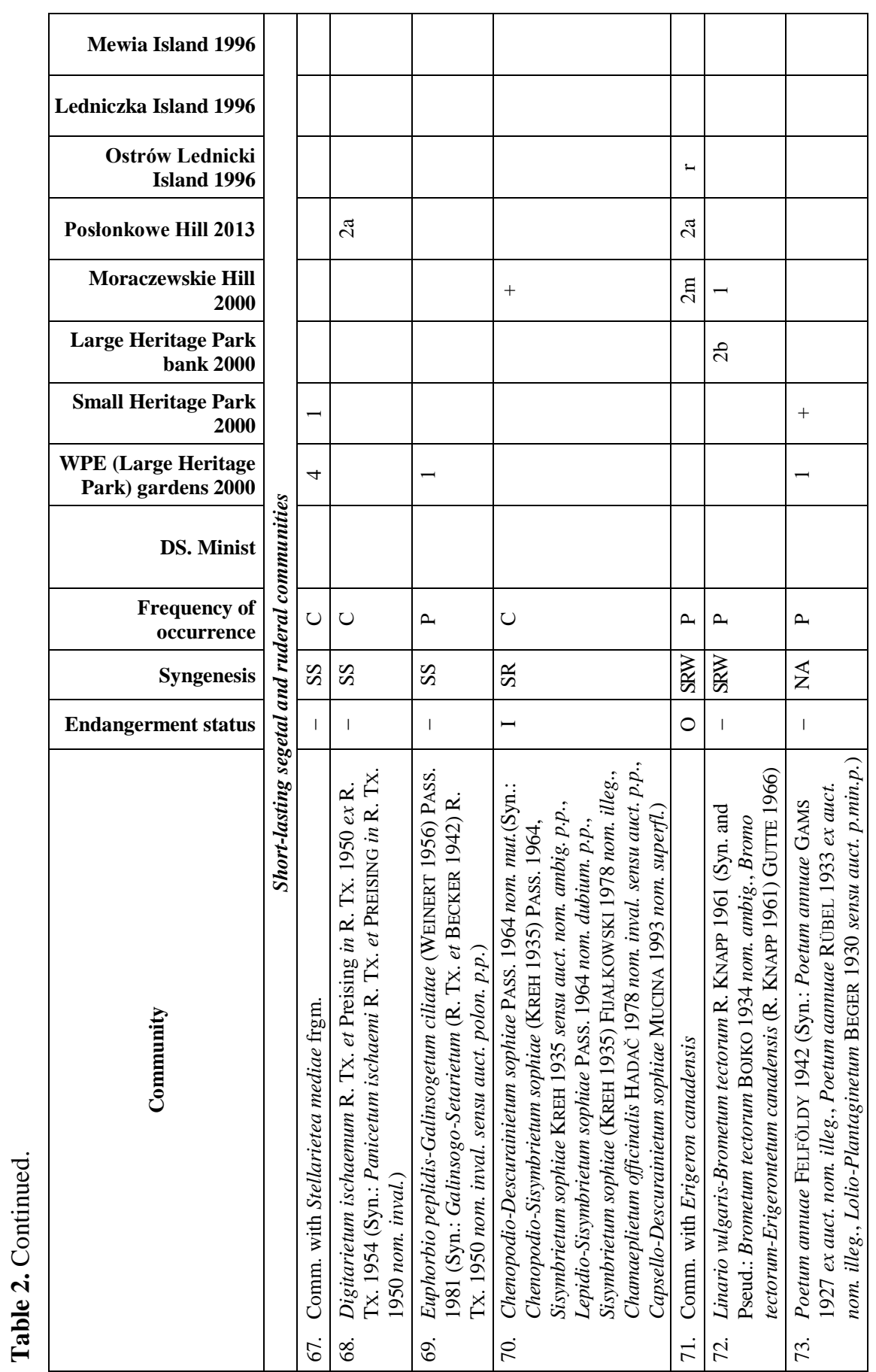




\begin{tabular}{|c|c|c|}
\hline Mewia Island 1996 & & \\
\hline Ledniczka Island 1996 & & तิ \\
\hline $\begin{array}{r}\text { Ostrów Lednicki } \\
\text { Island } 1996\end{array}$ & + & \\
\hline Posłonkowe Hill 2013 & & \\
\hline $\begin{array}{r}\text { Moraczewskie Hill } \\
2000\end{array}$ & & \\
\hline $\begin{array}{r}\text { Large Heritage Park } \\
\text { bank } 2000\end{array}$ & & \\
\hline $\begin{array}{r}\text { Small Heritage Park } \\
2000\end{array}$ & - & \\
\hline $\begin{array}{l}\text { WPE (Large Heritage } \\
\text { Park) gardens } 2000\end{array}$ & & \\
\hline DS. Minist & & \\
\hline $\begin{array}{r}\text { Frequency of } \\
\text { occurrence }\end{array}$ & $a$ & 0 \\
\hline Syngenesis & 竧 & $x$ \\
\hline Endangerment status & 1 & 1 \\
\hline है & 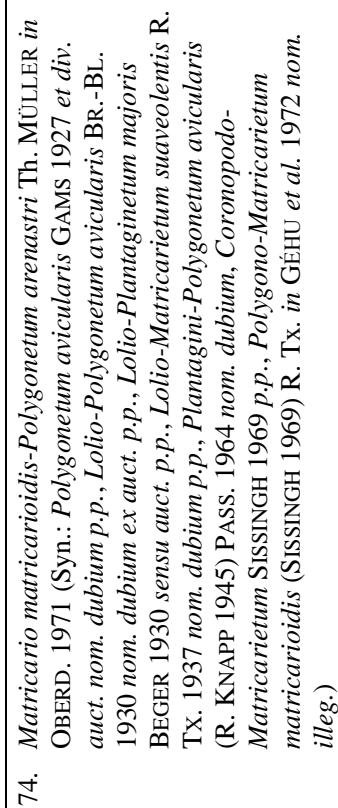 & 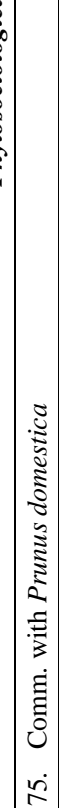 \\
\hline
\end{tabular}




\begin{tabular}{|c|c|c|c|c|c|c|c|c|c|c|c|c|c|c|c|c|c|c|}
\hline \multirow[t]{2}{*}{ Mewia Island 1996} & $b^{\circ}$ & $\stackrel{\infty}{\dot{f}}$ & 3 & 3 & $\stackrel{\infty}{\infty}$ & & $\stackrel{\infty}{m}$ & : & 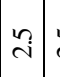 & \begin{tabular}{l|l}
$n$ & $\infty$ \\
$\vartheta$
\end{tabular} & & $\stackrel{\ominus}{\varrho}$ & ?ె & $\underline{2}$ & & $\sqrt[n]{2}$ & $\stackrel{n}{i}$ & \\
\hline & 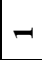 & $m$ & $\hat{n}$ & $n$ & $m$ & & $m$ & $\simeq$ & $\mathrm{N}$ & $m \mid n$ & & $\infty$ & $n$ & 2 & & $\mathrm{~N}$ & 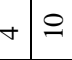 & \\
\hline \multirow{2}{*}{$\begin{array}{r}\text { Ledniczka Island } \\
1996\end{array}$} & $0^{\circ}$ & $\stackrel{\infty}{\circ}$ & $\stackrel{\check{\gamma}}{\vec{\gamma}}$ & $\begin{array}{l}0 \\
\stackrel{0}{-}\end{array}$ & 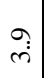 & $\stackrel{0}{-}$ & $\hat{\infty}$ & 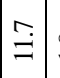 & 2 & $\stackrel{i}{\exists}$ & & 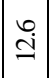 & 9 & $\stackrel{?}{I}$ & & & $\hat{ल} \vec{\sigma}$ & $\stackrel{-}{-}$ \\
\hline & - & F & 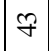 & \pm & $\nabla$ & - & $a$ & $\simeq$ & $N$ & -7 & & $\cong$ & N & $\stackrel{\infty}{\infty}$ & & & $+\stackrel{0}{\circ}$ & - \\
\hline \multirow{2}{*}{$\begin{array}{r}\text { Ostrów Lednicki } \\
\text { Island } 1996\end{array}$} & $s^{\circ}$ & 先 & \begin{tabular}{|l|}
$n$ \\
$\infty$ \\
$\infty$ \\
\end{tabular} & $\stackrel{n}{=}$ & $\stackrel{n}{q}$ & $\stackrel{\circ}{-}$ & $n$ & $\mid \begin{array}{l}n \\
\infty\end{array}$ & $\because$ & 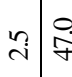 & & $\dddot{n}$ & $\stackrel{i}{i}$ & $\stackrel{2}{\infty}$ & $\stackrel{-}{-}$ & $n$ & \begin{tabular}{l|l}
$\stackrel{i}{i}$ & $\infty$ \\
\end{tabular} & $\stackrel{-}{\circ}$ \\
\hline & - & 8 & 5 & $\theta$ & $a$ & N & $=$ & $=$ & $m$ & n & & 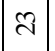 & 으 & $n$ & a & - & +0 & $a$ \\
\hline \multirow{2}{*}{$\begin{array}{r}\text { Posłonkowe Hill } \\
2013\end{array}$} & $0^{\circ}$ & 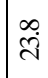 & 官 & $\begin{array}{l}0 \\
0 \\
2 \\
0\end{array}$ & F & $\stackrel{\infty}{0}$ & $\stackrel{0}{0}$ & $\hat{n}$ & $\exists$ & 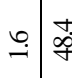 & & $\stackrel{n}{=}$ & $\stackrel{\infty}{\circ}$ & $\frac{m}{\lambda}$ & & $\stackrel{\infty}{\circ}$ & & \\
\hline & $=$ & নे & 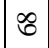 & 2 & $n$ & - & $\infty$ & $r$ & $n$ & $\alpha$ in & & \pm & -1 & ป & & - & & \\
\hline \multirow{2}{*}{ Moraczewskie $\underset{2000}{H i l l}$} & $b^{\circ}$ & $\stackrel{\sim}{=}$ & 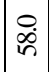 & $\stackrel{\grave{\lambda}}{\vec{\lambda}}$ & $\stackrel{+}{\infty}$ & $\hat{0}$ & $\stackrel{\dot{q}}{ }$ & 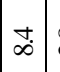 & $\begin{array}{l}\infty \\
i\end{array}$ & 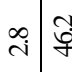 & & $+\infty$ & & రి. & & & & \\
\hline & - & 0 & $\infty$ & $\bar{m}$ & $\simeq$ & - & $r$ & $\simeq$ & t & +8 & & $\simeq$ & & $\infty$ & & & & \\
\hline \multirow{2}{*}{$\begin{array}{r}\text { Large Heritage Park } \\
\text { bank } 2000\end{array}$} & $0^{\circ}$ & $\ddot{g}$ & 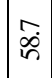 & $\stackrel{0}{\stackrel{1}{*}}$ & $\hat{\sigma}$ & 9 & $\tilde{\sigma}$ & $\hat{\varrho}$ & $\widehat{i}$ & 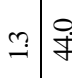 & & $\stackrel{\circ}{\rightarrow}$ & & $\hat{\dot{p}}$ & & & & \\
\hline & - & 은 & 寸 & $\because$ & $n$ & - & in & $\infty$ & $\sim$ & $-m$ & & $m$ & & $\approx$ & & & & \\
\hline \multirow{2}{*}{$\begin{array}{r}\text { Small Heritage Park } \\
2000\end{array}$} & $b^{\circ}$ & $\stackrel{+}{\ddot{2}}$ & $\stackrel{m}{q}$ & 苞 & $\stackrel{\partial}{\dot{I}}$ & 8 & $\stackrel{\varrho}{=}$ & $\stackrel{9}{=}$ & $\because$ & $\hat{q}$ & $\begin{array}{l}3 \\
\vdots\end{array}$ & $\stackrel{\circ}{\circ}$ & & ते & & & $\stackrel{n}{e}$ & \\
\hline & - & $a$ & $m$ & $=$ & 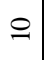 & $\nabla$ & $\infty$ & $\infty$ & - & ה & - & 0 & & $\mathscr{0}$ & & & $N-$ & \\
\hline \multirow{2}{*}{$\begin{array}{r}\text { WPE (Large } \\
\text { Heritage Park) } \\
\text { gardens 2000 }\end{array}$} & $0^{\circ}$ & ন & भิ & 守 & $\stackrel{n}{2}$ & $\begin{array}{l}1 \\
\infty\end{array}$ & $\vec{\nabla}$ & $\infty$ & $\stackrel{J}{i}$ & $\begin{array}{l}\infty \\
\infty \\
\infty\end{array}$ & & $\stackrel{\infty}{\exists}$ & & $\frac{\infty}{\dot{m}}$ & & & $\cong$ & \\
\hline & - & $\infty$ & $\ddot{m}$ & $\vec{\lambda}$ & $\cong$ & - & 0 & - & $a$ & $m$ & & 으 & & $\bar{\lambda}$ & & & - & \\
\hline \multirow[t]{2}{*}{ 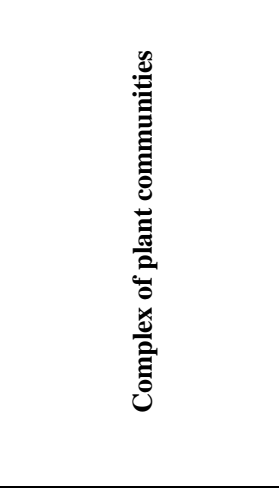 } & & 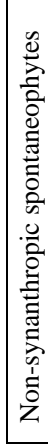 & $\mid$ & 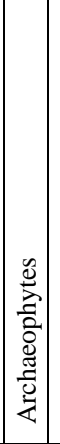 & 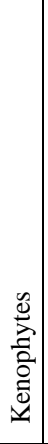 & 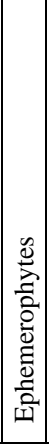 & 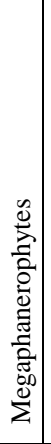 & 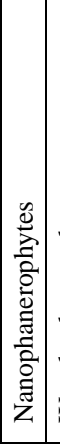 & 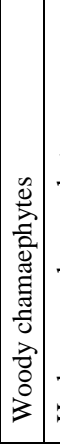 & 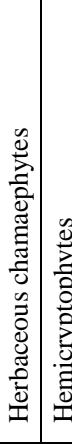 & 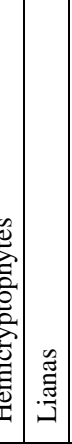 & 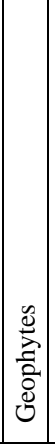 & 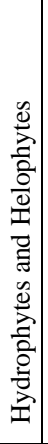 & 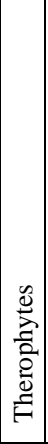 & 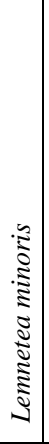 & 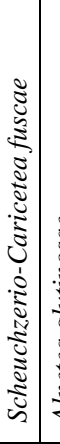 & 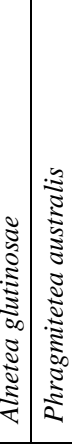 & 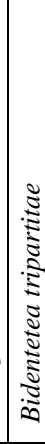 \\
\hline & & & \multicolumn{4}{|c|}{ GHG } & \multicolumn{8}{|c|}{ Life forms } & \multicolumn{4}{|c|}{ SG } \\
\hline
\end{tabular}




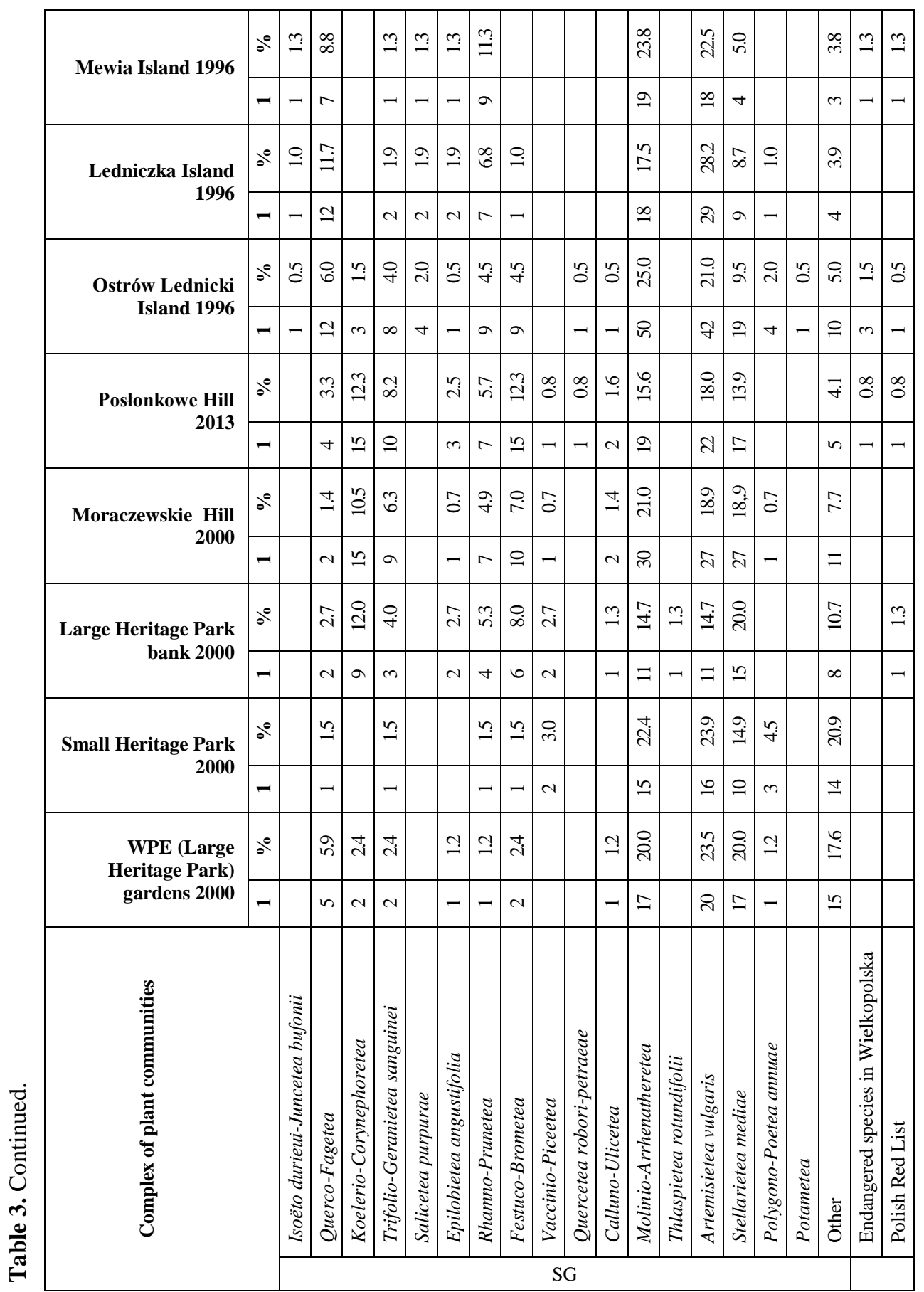




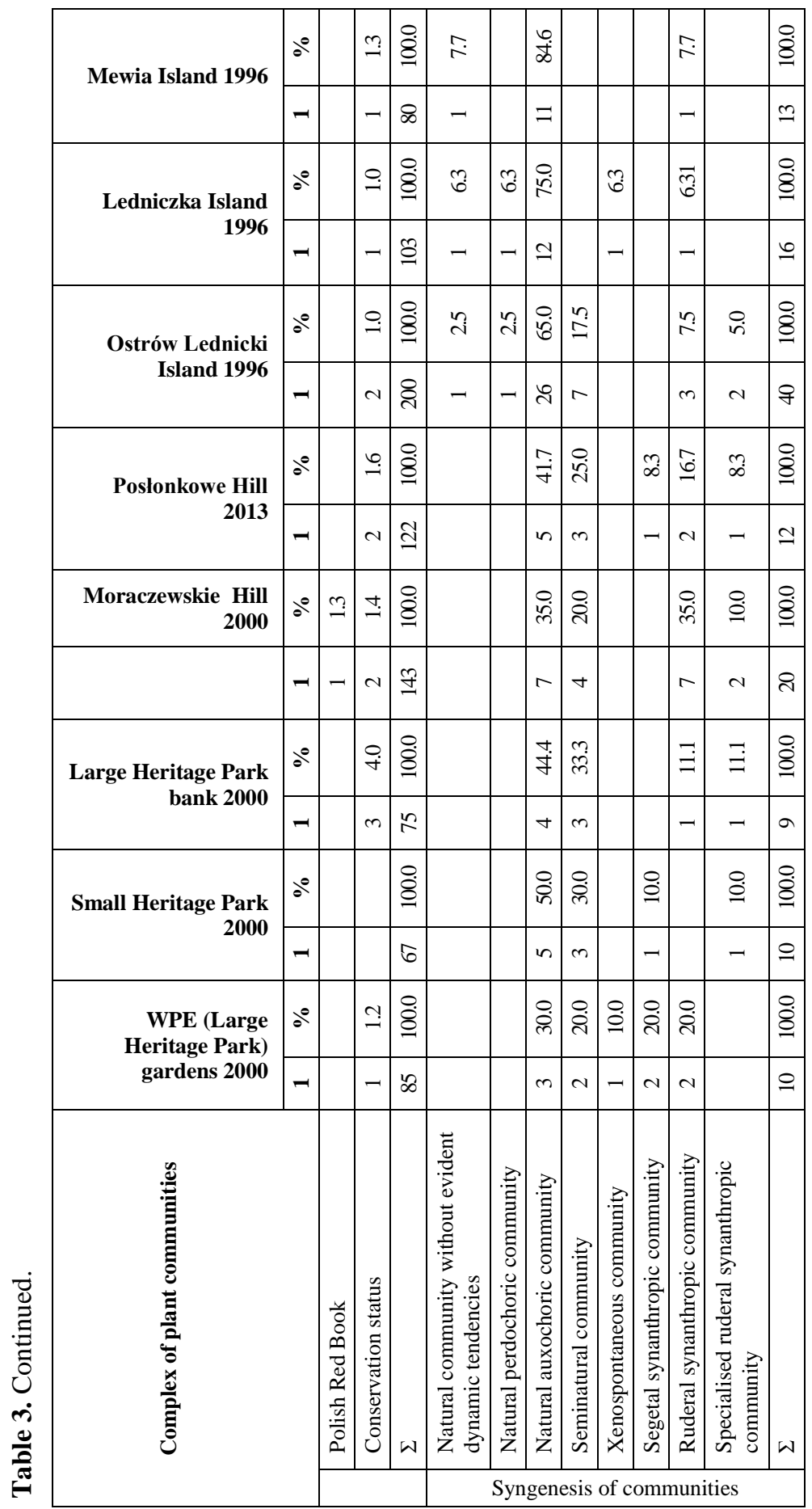


Bees were sampled systematically (throughout the respective growing seasons) over five years (1994-1998) and occasionally (approximately twice per growing season) for another five years from 2003 to 2007. The details of the sampling design varied from site to site:

- Wielkopolski Ethnographic Park (Large Heritage Park): bees were sampled systematically in 1994-1998 by using a sweep net (stalking) and by trapping them in Moericke colour traps. Bee density was also assessed on the hill with the escarpment in 1994-1996 from May to August (once or twice a month).

- Small Heritage Park: bees were sampled in 1996-1998 using Moericke pitfall traps and by stalking.

- Posłonkowe Hill: qualitative stalking of bees was carried out from April to August in 2003-2007.

- Moraczewskie Hill: bees were sampled using Moericke pitfall traps and by stalking in 1997-1998.

- Ostrów Lednicki Island: bees were sampled using Moericke pitfall traps and a sweep net (stalking), and bee density was assessed in 1994-1996.

- Ledniczka Island: bees were sampled using Moericke traps in 1994-1996. The sweep net was used on one occasion (29 April 1994).

- Mewia Island: bees were sampled into Moericke pitfall traps in 1994-1996. The sweep net was used on one occasion (29 April 1994).

Apart from the sites listed above, bee sampling by sweeping was carried out sporadically at Waliszewo (a grassland surrounded by a pine forest, 12 June 1994), Dziekanowice (a village outside the Heritage Park, 20 August 2003) and Imiołki (a meadow on the north-western shore of Lake Lednica and a motte-and-bailey castle with a thermophilous grassland).

Insects were captured in Moericke colour traps, i.e. white bowls filled with a preservative liquid. The liquid was a mixture of water (95\%), ethylene glycol $(4.8 \%)$ and surfactant $(0.2 \%)$. Such bowls were used in the Wielkopolska Ethnographic Park, the Small Heritage Park and on Moraczewskie Hill from March to early October. The sweep net was also used at all sites, with bees searched for on flowers and in favourite nesting sites during field visits, most often at monthly intervals from March-April to September-October. Most samples collected on the islands were captured in Moericke traps. Sets of six bowls were placed directly on the ground at each site. On the small and densely vegetated islands, a few (3) bowls were hung on tree or shrub branches at a height of approximately $1.5 \mathrm{~m}$. The bowls were emptied at weekly intervals and the insects were preserved in a 70\% ethyl alcohol solution and subsequently pinned.

Bee density was assessed using linear transects or belts (BANASZAK 1980). Bees were recorded in belts $1 \mathrm{~m}$ wide and $200 \mathrm{~m}$ long: an area of $200 \mathrm{~m}^{2}$ constituted one sample. 
Assessments were conducted when the weather conditions were favourable for bee flights, i.e. at air temperatures of at least $20^{\circ} \mathrm{C}$ and a slight wind. The plant species used by the bees were recorded on the same occasion, so that the list of food plants could be made more complete. Density was assessed on Ostrów Lednicki Island and at the site by the lake.

Bee species diversity and richness was analysed by constructing a function of the number of species (rarefaction curves) against the number of samples (sample-based rarefaction) (GOTELLI \& COLWELL 2001) using the MauTau estimator (COLWELL et al. 2004). The calculations were carried out in EstimateS (COLWELL 2006).

The structural diversity of the bee assemblages in the study habitats was determined by the NMDS ordination method (multi-dimensional scaling). Bray-Curtis dissimilarity was used to assess the degree of similarity between individual samples because of its close relation to changes in assemblage composition (FAITH et al. 1987). Samples consisting of at least five individuals were included in the ordination analysis. The statistical significance of differences between assemblages (groups of samples) was determined using the multiresponse permutation procedure (MRPP), which tests the hypothesis of no differences in species composition between the sites being compared (ZIMMERMAN et al. 1985, BONNER et al. 2009). The MRPP method uses a Bray-Curtis dissimilarity matrix to compare mean intragroup and intergroup dissimilarity. When the null hypothesis was rejected, comparisons according to the MRPP method were carried out for each pair of habitats to determine the significance of differences between the habitats.

To determine the species responsible for the lack of homogeneity between clusters of samples identified by NMDS analysis, indicator values (IndVal) were calculated for each species recorded in these groups (DUFRÊNE \& LEGENDRE 1997). The IndVal method allows one to calculate the strength of association of a particular species with a given habitat by analysing the constancy of its occurrence across samples and relative density. Species strongly associated with a given habitat are those whose IndVal exceeded 0.25. The significance of indicator values was assessed with a permutation test with 10000 repetitions. These analyses were conducted in the statistical package R (RDCT 2008) using the vegan (OKSANEN et al. 2011) and labdsv (ROBERTS 2010) libraries.

\section{Characteristics of the study sites}

\section{A. Environs of Lake Lednica}

1. The Wielkopolska Ethnographic Park (the Large Heritage Park) is situated on the outskirts of the village of Dziekanowice in the basin of Lake Lednica. It is one of the largest open-air museums in Poland, exhibiting historical wooden buildings relocated from sites within the historical Wielkopolska region and copies of brick buildings. Established in 1975, the Heritage Park occupies an area of 21 ha. To date, more than 50 reconstructed buildings from the $19^{\text {th }}$ and $18^{\text {th }}$ centuries are on display. Adjoining the 
households are small gardens with traditional plants (ornamental plants, herbs and vegetables), orchards and fields representing attractive locations for bees, including the clay and timber walls of buildings and the straw and reed roofing. Large numbers of tourists visit the museum. The potential natural vegetation is Galio sylvatici-Carpinetum oak-hornbeam forest. A total of 85 species of vascular plants have been recorded in the area, almost $52 \%$ of which are native species, mostly apophytes. The vast majority of non-native species are archaeophytes. The two co-dominant life forms are hemicryptophytes (nearly 39\%) and therophytes (nearly 32\%), with a significant contribution of phanerophytes (over 15\%). There are representatives of 12 phytosociological classes, the most numerous being taxa of mainly ruderal, tall herbs of the Artemisietea class (23.5\%), field taxa of the Stellarietea mediae class (20\%) and meadow and pasture taxa of the Molinio-Arrhenatheretea class (20\%). Blackcurrant (Ribes nigrum) was the only legally protected species identified. The largest group among the 10 plant communities found is that of natural auxochoric communities (3), while semi-natural, segetal and ruderal communities are represented by two of each. In terms of area occupied, the dominant communities are those of field weeds, usually species-impoverished. Legally protected habitat types (2) comprise a small-sized patch of a mesic Arrhenatheretum elatioris meadow (6510) and tall herbs (6430).

Immediately adjoining the Park is an escarpment with a southerly exposure. The habitat is poor there. The potential natural vegetation is Querco roboris-Pinetum mixed coniferous forest and Leucobryo-Pinetum mesic coniferous forest. A total of 75 plant species have been identified, of which native forms, mainly apophytes, account for $72.4 \%$. The dominant anthropophytes by far are archaeophytes. With regard to life forms, there is a co-dominance of hemicryptophytes (over 39\%) and therophytes (over $33 \%$ ). Phanerophytes are mostly shrubs. Of the 12 phytosociological classes present, the largest group is that of edificators of the Stellarietea mediae class (almost 22\%). Other abundant classes include Molinio-Arrhenatheretea (almost 16\%), Artemisietea (almost $12 \%)$ and Koelerio-Corynephoretea and Festuco-Brometea, i.e. grassland species, which together account for more than $20 \%$. "Special care" species include some listed in the Polish Red Book: Sorbus intermedia (at an anthropogenic site), and on the Polish Red List: Vernal Sedge Carex praecox; legally protected species include Dwarf Everlast Helichrysum arenarium, Spiny Restharrow Ononis spinosa and Swedish Whitebeam Sorbus intermedia. The most abundant of the 9 plant communities are natural auxochoric (4) and semi-natural (3) communities. With regard to area occupied, the dominant community is species-impoverished psammophilous grassland. Protected habitat types (3) include psammophilous grasslands (2330) and $(* 6120)$ a mesic meadow (6510). 
Bees were collected from the entire area of the Heritage Park, from the escarpment and an eminence with a group of old windmills, both covered with thermophilous grassland. The most important food plants are Taraxacum officinale, Ballota nigra, Potentilla argentea, Senecio vernalis, Rosa canina, Veronica chamaedrys, Anchusa officinalis, Centaurea stoebe, Medicago falcata, Rubus idaeus, Melilotus alba, Cirsium arvense, Cynoglossum officinale, Fragaria vesca, Carduus acanthoides and Ononis spinosa.

2. The Small Heritage Park, situated north of the village of Dziekanowice and $1.5 \mathrm{~km}$ distant from the Wielkopolska Ethnographic Park. This small area (1.9 ha) of a former mediaeval settlement, between the lake shore and arable fields, hosts several historical wooden buildings and reconstructed ones with typical country gardens; there are also orchards. A mediaeval settlement of iron-workers and blacksmiths was located there from the $9^{\text {th }}$ to the $13^{\text {th }}$ century. Later, there was a cemetery on the northern outskirts. A total of 1500 graves have been discovered here to date (CHOJNACKA \& RASZKA 2007).

The potential natural vegetation is Galio sylvatici-Carpinetum oak-hornbeam forest. Sixty-seven species of vascular plants have been identified, almost $63 \%$ of which are native, mainly apophytes. Among the anthropophytes, there is a slight predominance of archaeophytes. The most numerous life forms are hemicryptophytes (over 40\%), with abundant therophytes (almost 24\%) and phanerophytes (almost 24\%). The 11 phytosociological classes identified are characterised by the co-dominance of tall herb taxa of the Artemisietea class (almost 24\%), meadow and pasture taxa of the MolinioArrhenatheretea class (almost 22\%) and segetal taxa of the Stellarietea mediae class (almost 15\%). A feature of note is the large percentage (almost 21\%) of species without a distinct phytosociological affiliation. Ten plant communities were found, natural auxochoric ones (5) being the prevailing type. The dominant type of area occupied is meadows and pastures, and particularly phytocoenoses resistant to trampling (intensive tourist pressure): Lolio-Cynosuretum and Lolio-Plantaginetum. Protected habitats (2) are mostly small patches of mesic Arrhenatheretum elatioris meadow (6510) and tall herbs (6430).

The most important food plants for bees are Tussilago farfara, Salix cinerea, Taraxacum officinale, Lamium album, L. purpureum, Cirsium arvense, Convolvulus arvensis, Glechoma hederacea, Malus domesticus, Prunus domestica, Medicago sativa, Ribes rubrum and Symphytum officinale.

3. Posłonkowe Hill is an eminence in a field landscape near a road not far from the railway station in Lednogóra, at the border of the LLP buffer zone. Nearby, there are small 
wooded areas, mostly with pines. This rich site of xerothermal flora is named after an abundant population of the Common Rockrose Helianthemum nummularium (TOBOLSKI 1996). The site is heterogeneous, comprising formerly arable land with some new ruderal and xerothermophilous vegetation and some patches of xerothermal communities, which are becoming overgrown with tall herbs and tree and shrub undergrowth. The potential natural vegetation is the poorer series of Galio sylvaticiCarpinetum oak-hornbeam forest. There is an illegal sand quarry nearby. A total of 122 plant species have been identified, $79 \%$ of which are native forms, mainly apophytes. Archaeophytes make up the largest group among the anthropophytes. The dominant life-forms are hemicryptophytes (almost 50\%), with a significant contribution from therophytes (almost 22\%), geophytes (over 10\%) and phanerophytes. The most numerous of the 13 phytosociological classes present there include ruderal and tall herb taxa of the Artemisietea class (almost 18\%), meadows and pastures of the MolinioArrhenatheretea class (16\%), segetal taxa of the Stellarietea mediae class (over 13\%), as well as thermophilous tall herbs of the Trifolio-Geranietea class and xerothermal grasslands of the Festuco-Brometea class (12.6\% each). There are rich sites of the protected Dwarf Everlast Helichrysum arenarium, Common Cowslip Primula veris ssp. veris. Stiff Hedgenettle Stachys recta is regionally endangered. There are twelve plant communities, with prevalent natural and auxochoric ones (5), and three semi-natural communities. The largest area is occupied by partly developed xerothermal grassland communities. Psammophilous grasslands (2330) are a protected habitat; since communities with Festuco-Brometea are species-impoverished, they were not included among Directive-covered habitats.

The main food plants are Tussilago farfara, Knautia arvensis, Potentilla sp. div., Centaurea stoebe, C. scabiosa, Veronica spicata, V. chamaedrys, Fragaria vesca, Crataegus monogyna, Sedum acre, Thymus pulegioides, Helichrysum arenarium, Galium verum, Salix caprea and Gagea pratensis.

4. Moraczewskie Hill by the Poznań-Gniezno road is an eminence with three windmills. This steep escarpment with slopes up to $40^{\circ}$ and a south-westerly exposure has xerothermal vegetation. Owing to the attractive landscape as well as its natural and cultural value, the hill was put forward for legal protection as an ecological interest site (ТовOLSKI 1991). The potential natural vegetation is a poor series of Galio sylvaticiCarpinetum oak-hornbeam forest or Querco roboris-Pinetum mixed coniferous forest. A total of 143 plant species have been identified, of which over $69 \%$ are native, mainly apophytes. The most numerous group among the anthropophytes is that of early introductions (archaeophytes). The dominant life forms are hemicryptophytes (over $46 \%$ ), with a significant proportion of taxa exhibiting short life cycles (almost 27\%), 
phanerophytes (over 13\%) and geophytes (over 8\%). There are 12 phytosociological classes, the most abundant ones including representatives of fields with MolinioArrhenatheretea (almost 21\%), segetal habitats of the Stellarietea mediae class (almost 19\%), ruderal Artemisietea tall herbs (almost 19\%) and grassland habitats of the Koelerio-Corynephoretea and Festuco-Brometea classes (collectively 17.5\%). Protected species include the Dwarf Everlast Helichrysum arenarium and Common Cowslip Primula veris ssp. veris. As many as 9 of the 20 plant communities are ruderal, with an abundance of natural auxochoric (7) and semi-natural (4) habitats. The largest area is covered by impoverished xerothermal and psammophilous grassland communities. The following three protected habitat types are represented at the site: psammophilous grasslands (2330), mesic meadows (6510) and tall herbs (6430).

The food plants of bees identified at this site include Anchusa officinalis, Armeria elongata, Berteroa incana, Carduus acanthoides, Cirsium arvense, Coronilla varia, Crataegus monogyna, Cynoglossum officinale, Echium vulgare, Helichrysum arenarium, Jasione montana, Knautia arvensis, Lotus corniculatus, Medicago falcata, M. sativa, Melilotus alba, M. officinalis, Potentilla anserina, P. argentea, Prunus spinosa, Senecio jacobaea, S. vernalis, Taraxacum officinale and Vicia villosa.

B. Islands on Lake Lednica

1. Ostrów Lednicki is the biggest of the islands that we investigated. Approximately $470 \mathrm{~m}$ long and $250 \mathrm{~m}$ wide, it has an area of $7.5 \mathrm{ha}$. It lies $150 \mathrm{~m}$ from the eastern shore of the lake and $370 \mathrm{~m}$ from the western shore. The island can be described as very heterogeneous, both in terms of terrain (with height differences of the order of $10 \mathrm{~m}$ ) and human impact (including an archaeological excavation and exhibition site, a ferry haven and a network of paths). The southern part of the island encompasses a $150 \times 190$ $\mathrm{m}$ former fortified settlement of the concave type and a $6 \mathrm{~m}$ high wall (HENSEL \& HILCZER-KURNATOWSKA 1972). It is popular with tourists arriving by ferry. Herbaceous plants grow within the settlement. The plants are mown from time to time near some parts of the wall and in the depression. The shores of the islands are covered with trees and shrubs, mainly Alnus glutinosa, Salix alba and S. cinerea and there is a belt of Phragmitetum communis reeds and tall herbs with Lysimachia vulgaris, Petasites hybridus, Lythrum salicaria, Cirsium oleraceum and Scrophularia umbrosa. The northern part of the island supports a small mown meadow and an area of shrubs consisting mainly of Crataegus monogyna, Pyrus communis and Prunus spinosa. The potential natural vegetation comprises Galio sylvatici-Carpinetum oak-hornbeam forest, and riparian forests with elm and ash (Querco-Ulmetum), ash and alder (FraxinoAlnetum), willow (Salicetum albae) as well as a fertile alder forest (Carici elongataeAlnetum) and communities of aquatic plants and reeds. CELKA (1998) identified 197 
plant species on the island, which can be attributed to the size of the island and habitat diversity. Our investigations revealed the presence of 200 species. Indigenous species, mainly apophytes, account for more than $83 \%$, with spontaneophytes also occurring quite abundantly at over $35 \%$. The predominant non-native taxa are archaeophytes. Hemicryptophytes account for more than $47 \%$, and there are also significant contributions from therophytes (almost 19\%), phanerophytes (13\% overall) and geophytes $(11.5 \%)$. The plant species represent 20 phytosociological classes, showing the co-dominance of meadow and pasture edificators of the Molinio-Arrhenatheretea class (25\%) and tall herbs of the Artemisietea class (over 20\%), and other fairly abundant classes including field vegetation of the Stellarietea mediae class (over 9\%) and reed vegetation of the Phragmitetea class (over 8\%). Regionally endangered species comprise the Water Germander Teucrium scordium, Golden Oat Grass Trisetum flavescens and Saw-Leaved Speedwell Veronica teucrium, while the Common Cowslip Primula veris ssp. veris is a protected species. As many as 40 distinct plant communities were found, the majority of which (over 69\%) were natural, almost exclusively auxochoric. The largest area is occupied by woodland and scrub, and tall herbs; there is also an abundant contribution of grass communities. Protected habitat types (5) are riparian forests $(* 91 \mathrm{E} 0)$, mesic meadows $(6510)$, tall herbs $(6430)$, communities of shore-associated therophytes (3270) and aquatic plants (3150).

The main food plants of bees in this area are Primula veris, Salix alba, S. cinerea, Taraxacum officinale, Prunus spinosa, Ballota nigra, Vicia sepium, Anchusa officinalis, Veronica chamaedrys, Cirsium sp. div., Lathyrus pratensis, Lysimachia vulgaris, Potentilla arenaria, Crataegus monogyna, Lotus corniculatus, Anthriscus sylvestris, Malva alcea, Rubus caesius and Medicago falcata.

2. Ledniczka, approximately 1.3 ha in area, is situated in the western part of the lake, approximately $200 \mathrm{~m}$ south-west of Ostrów Lednicki Island and $100 \mathrm{~m}$ from the western lake shore. The centre of the island is occupied by a former fortified settlement of the cone type, with an $18^{\text {th }}$ century wooden bell tower at the top. About 40 years ago the island was economically managed, supporting small gardens and orchards, the contemporary remnants of which include mainly Prunus domestica shrubs. Now the settlement and the entire island are overgrown with trees and shrubs, mainly Alnus glutinosa, Salix alba, Ulmus laevis, Cornus sanguinea and Rosa canina. The fringe of the island supports a belt of reed bed. The potential natural vegetation consists of riparian forests with elm and ash Querco-Ulmetum, ash and alder Fraxino-Alnetum and Salicetum albae. CELKA (1998) recorded 82 species of vascular plants, and our investigations revealed the presence of 103 taxa, 82\% of which are native, with 50\% each of apophytes and non-synanthropic spontaneophytes. Among anthropophytes, 
archaeophytes are abundant. The dominant life forms are hemicryptophytes (45\%), with phanerophytes (shrubs being more frequent) accounting for $21 \%$ overall. Therophytes $(17 \%)$ and geophytes (12\%) are also quite numerous. The flora can be divided into 14 phytosociological classes, the most abundant of which are the characteristic Artemisietea (27\%), followed by Molinio-Arrhenatheretea (17\%) and Querco-Fagetea (10\%). The Common Cowslip Primula veris ssp. veris is legally protected. As many as 14 (almost 88\%) of the 16 plant communities identified are indigenous, mainly auxochoric. The largest proportion of the area is covered by scrub, with a considerable proportion of tall herbs and younger stages of riparian forests. Protected habitats (4) include riparian forests $(* 91 \mathrm{E} 0,91 \mathrm{~F} 0)$, tall herbs $(6430)$ and clumps of shore-associated therophytes (3270).

The main food plants of bees are Salix alba, Hypericum perforatum, Malva alcea, M. sylvestris, Lysimachia vulgaris, Primula veris, Rosa canina, Rubus caesius, Crataegus monogyna, Prunus spinosa, Melilotus officinalis, Glechoma hederacea, Ballota nigra, Tussilago farfara, Cirsium arvense, C. oleraceum and Taraxacum officinale.

3. Mewia Island (Ptasia Island) is the smallest of the islands that we investigated ( $0.5 \mathrm{ha})$. It lies about $150 \mathrm{~m}$ from the eastern shore and approximately $250 \mathrm{~m}$ north of Ostrów Lednicki Island It used to be farmed, but now almost the entire island is overgrown with trees and shrubs, with only small clearings occupied by tall herbs. The dominant species is Alnus glutinosa, with contributions from Salix cinerea, S. purpurea and Rubus caesius. The fringe supports Phragmites and Carex reed/sedge beds. The potential natural vegetation is that of riparian forests: elm and ash Querco-Ulmetum, ash and alder Fraxino-Alnetum and fertile alder forest Carici elongatae-Alnetum. There are 80 species of vascular plants, nearly $90 \%$ of which are native, with a slight predominance of apophytes. Among anthropophytes, archaeophytes are more abundant. The dominant life forms are hemicryptophytes (almost 44\%), while phanerophytes account for almost $19 \%$ overall and are represented almost exclusively by shrubs. Therophytes (over 16\%) and geophytes (10\%) are also more abundant. Phytosociologically, the flora is represented by 12 classes, the most numerous being meadow and pasture taxa of the Molinio-Arrhenatheretea class (almost 24\%), tall herb taxa of the Artemisietea class (22.5\%), reed taxa of the Phragmitetea class (12.5\%) and bushes of the RhamnoPrunetea class (over 11\%). The Common Cowslip Primula veris ssp. veris is legally protected. Only one of the 13 plant communities is ruderal, the remaining ones being natural, almost exclusively auxochoric. With regard to the proportion of the area occupied, bushes, reed beds and tall herbs are co-dominants. Protected habitats (2) are represented by ash-alder riparian forest $(* 91 \mathrm{E} 0)$ and tall herbs (6430). 
The most important food plants for bees are Ficaria verna, Salix cinerea, S. purpurea, Prunus spinosa, Taraxacum officinale, Primula veris, Lamium purpureum, Ballota nigra, Crataegus monogyna and Cirsium arvense.

\section{RESULTS}

\section{Species diversity and dominance structure (patterns) in Apiformes}

During the fieldwork, we collected a total of 5159 individual bees, representing 161 species. A full list with a breakdown of individual counts per site can be found in Table 4 . The species identified in our study account for $34.2 \%$ of Poland's bee fauna.

Table 4. List of species and number of individuals of Apiformes found in the Lednica Landscape Park.

\begin{tabular}{|c|c|c|c|c|c|c|c|c|c|}
\hline Species & 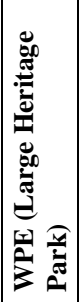 & 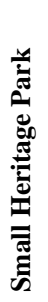 & 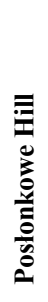 & 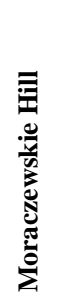 & 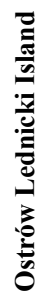 & 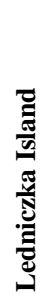 & 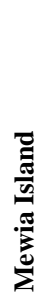 & 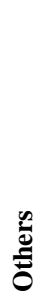 & 芯 \\
\hline \multicolumn{10}{|c|}{ Colletidae } \\
\hline 1. Colletes cunicularius (LINNAEUS, 1761) & 5 & & 1 & & & 2 & & & 8 \\
\hline 2. C. daviesanus SMITH, 1846 & 1 & 1 & & & 3 & & & & 5 \\
\hline 3. C. fodiens (FOURCROY, 1758) & & & 2 & & & & & 1 & 3 \\
\hline 4. Hylaeus angustatus (SCHENCK, 1859) & & & & & & 1 & & 1 & 2 \\
\hline 5. H. annularis (KIRBY, 1802) & 1 & & 2 & & & & & & 3 \\
\hline 6. H. communis NYLANDER, 1852 & 5 & 2 & & & & 13 & 4 & 2 & 26 \\
\hline 7. H. confusus NYLANDER, 1852 & & & & & 2 & 1 & 1 & & 4 \\
\hline 8. H. difformis (EVERSMANN, 1852) & 1 & & & & & 1 & & & 2 \\
\hline 9. H. gibbus SAUNDERS, 1850 & 2 & 1 & & 2 & & 1 & & & 6 \\
\hline 10. H. gracilicornis (MORAWITZ, 1867) & 1 & & & & & & & & 1 \\
\hline 11. H. moricei luteifrous (STRAND, 1909) & & & & & & 2 & & & 2 \\
\hline 12. H. pectoralis FÖRSTER, 1871 & & & & & & 1 & 12 & & 13 \\
\hline 13. H. punctulatissimus SмIтH, 1842 & & 1 & & & & & & & 1 \\
\hline \multicolumn{10}{|c|}{ Andrenidae } \\
\hline 14. Andrena alfkenella PERKINS, 1914 & & & & 2 & & 1 & & & 3 \\
\hline 15. A. apicata SMITH, 1847 & 18 & 3 & & 1 & 1 & 1 & 1 & & 25 \\
\hline 16. A. bicolor FABRICIUS, 1775 & 21 & 13 & 1 & 4 & 1 & 1 & 3 & & 44 \\
\hline 17. A. chrysosceles (KIRBY 1802) & & & & & & 1 & & & 1 \\
\hline 18. A. cineraria (LINNAEUS, 1758) & 3 & 5 & & & & 1 & & & 9 \\
\hline
\end{tabular}


Table 4. Continued.

\begin{tabular}{|c|c|c|c|c|c|c|c|c|c|}
\hline Species & 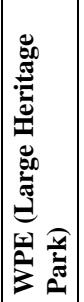 & 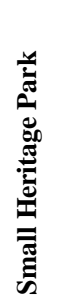 & 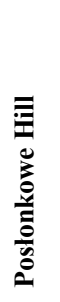 & 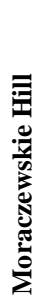 & 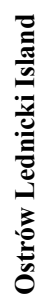 & 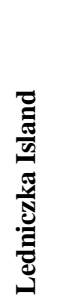 & 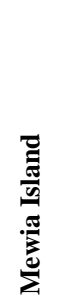 & $\frac{\mathscr{\omega}}{\tilde{\Xi}}$ & ڤ్ \\
\hline 19. Andrena clarkella (KIRBY, 1802) & 1 & 25 & & 1 & 4 & & 2 & & 33 \\
\hline 20. A. dorsata (KIRBY, 1802) & 8 & 1 & 1 & 2 & & 1 & 1 & & 14 \\
\hline 21. A. falsifica PERKINS, 1915 & 1 & & 8 & & & & & & 9 \\
\hline 22. A. flavipes PANZER, 1799 & 24 & 10 & 3 & 2 & 2 & 7 & & & 48 \\
\hline 23. A. floricola EVERSMANN, 1852 & & & & 1 & & & & & 1 \\
\hline 24. A. fucata SMITH, 1847 & 2 & & & & & 8 & & & 10 \\
\hline 25. A. fulva (SCHRANK, 1781) & 73 & 26 & & 4 & 9 & 5 & 12 & 2 & 131 \\
\hline 26. A. gravida IMHOFF, 1899 & 3 & 4 & & & & & 1 & & 8 \\
\hline 27. A. haemorrhoa (FABRICIUS, 1781) & 64 & 128 & 6 & 16 & 14 & 75 & 46 & 8 & 357 \\
\hline 28. A. hattorfiana (FABRICIUS, 1775) & & & 5 & & & & & & 5 \\
\hline 29. A. helvola (LINNAEUS, 1758) & 8 & 38 & & & 29 & 150 & 115 & & 340 \\
\hline 30. A. humilis IMHOFF, 1832 & & & & & & & & 1 & 1 \\
\hline 31. A. jacobi PERKINS, 1921 & 1 & 6 & & & & 4 & & & 11 \\
\hline 32. A. labiata SCHENCK, 1851 & 2 & 2 & 8 & & 1 & & & & 13 \\
\hline 33. A. minutula (KIRBY, 1802) & 7 & 4 & & 1 & & 1 & & & 13 \\
\hline 34. A. nasuta GIRAUD, 1863 & & & & & 16 & & & & 16 \\
\hline 35. A. nigroaenea (KIRBY, 1802) & 9 & 8 & 1 & & 3 & 5 & 2 & 1 & 29 \\
\hline 36. A. nitida (MÜLLER, 1776) & 4 & 56 & & & 13 & 26 & 10 & 5 & 114 \\
\hline 37. A. nycthemera IMHOFF, 1866 & 1 & 1 & & & & & & & 2 \\
\hline 38. A. ovatula (KIRBY, 1802) & 1 & & & & & & & & 1 \\
\hline 39. A. praecox (SCOPOLI, 1763) & 17 & 41 & & 28 & 12 & 67 & 2 & & 167 \\
\hline 40. A. subopaca NYLANDER, 1848 & 33 & 34 & 1 & 11 & 8 & 28 & 18 & 4 & 137 \\
\hline 41. A. synadelpha PERKINS, 1914 & 1 & 2 & & & 4 & 17 & 1 & 1 & 26 \\
\hline 42. A. tibialis (KIRBY, 1802) & 3 & 1 & & & 1 & 5 & 4 & & 14 \\
\hline 43. A. vaga PANZER, 1799 & 25 & 2 & & & 20 & 27 & 22 & & 96 \\
\hline 44. A. ventralis IMHOFF, 1832 & 5 & & 8 & & & & & & 13 \\
\hline 45. A. viridescens VIERECK, 1916 & & & & & 1 & & & & 1 \\
\hline 46. Panurgus calcaratus (SCOPOLI, 1763) & 1 & & & & & & & & 1 \\
\hline \multicolumn{10}{|c|}{ Halictidae } \\
\hline 47. Rhophitoides canus (EVERSMANN, 1852) & 8 & & & & & & & & 8 \\
\hline 48. Halictus maculatus SMITH, 1848 & 16 & & 3 & & & & & 2 & 21 \\
\hline 49. H. quadricinctus (FABRICIUS, 1776) & 9 & & 9 & & & & & & 18 \\
\hline 50. H. rubicundus (CHRIST, 1791) & 1 & & 1 & 1 & & & & 1 & 4 \\
\hline 51. H. sexcinctus (FABRICIUS, 1775) & 2 & & 12 & & 2 & & 1 & 6 & 23 \\
\hline 52. Seladonia confusa (SMITH, 1853) & 6 & 2 & 2 & 2 & & & & 2 & 14 \\
\hline 53. S. leucahenea $($ EBMER, 1972$)$ & & & 3 & & & & & & 3 \\
\hline
\end{tabular}


Table 4. Continued.

\begin{tabular}{|c|c|c|c|c|c|c|c|c|c|}
\hline Species & 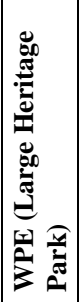 & 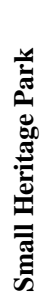 & 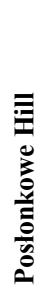 & 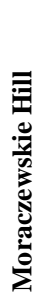 & 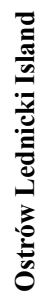 & 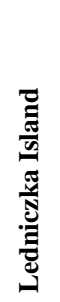 & 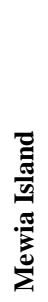 & $\frac{\mathscr{a}}{\bar{\Xi}}$ & ڤ \\
\hline 54. Seladonia subaurata (RossI, 1792) & 1 & & & & & & & & 1 \\
\hline 55. S. tumulorum (LINNAES, 1758) & 20 & 10 & 1 & 4 & 6 & 1 & & & 42 \\
\hline 56. Lasioglossum lativentre (SCHENCK, 1853) & & & & & & & 1 & & 1 \\
\hline 57. L. leucozonium (SCHRANK, 1781) & 4 & 1 & & & & & & 7 & 12 \\
\hline 58. L. quadrinotatum (KIRBY, 1802) & & 4 & & 1 & 1 & 5 & 5 & & 16 \\
\hline 59. L. sexnotatum (KIRBY, 1802) & 8 & 53 & 1 & 3 & 7 & 14 & 6 & & 92 \\
\hline 60. L. subfasciatum (IMHOFF, 1832) & 14 & 4 & & 1 & 5 & 19 & 2 & & 45 \\
\hline 61. L. xanthopus (KIRBY, 1802) & & & 1 & & 2 & 1 & & & 4 \\
\hline 62. L. zonulum (SMITH, 1848) & & & & & 1 & & & & 1 \\
\hline 63. Evylaeus calceatus (SCOPOLI, 1763) & 75 & 75 & 1 & 17 & 94 & 43 & 14 & 3 & 322 \\
\hline 64. E. intermedius (SCHENCK, 1868) & & & & & & 2 & & & 2 \\
\hline 65. E. leucopus (KIRBY, 1802) & 3 & 1 & 1 & 1 & & & & & 6 \\
\hline 66. E. lucidulus (SCHENCK, 1861) & 3 & & & & & 1 & & & 4 \\
\hline 67. E. minutissimus (KIRBY, 1802) & 1 & & & & & 4 & 2 & & 7 \\
\hline 68. E. morio (FABRICIUS, 1793) & 48 & 1 & & 3 & 14 & 93 & & & 159 \\
\hline 69. E. nitidiusculus (KIRBY, 1802) & 4 & 5 & 8 & & 4 & 2 & & & 23 \\
\hline 70. E. nitidulus (FABRICIUS, 1804) & & 1 & & & 1 & & & & 2 \\
\hline 71. E. parvulus (SCHENCK, 1853) & & 2 & & & 1 & 12 & & & 15 \\
\hline 72. E. pauxillus (SCHENCK, 1853) & 14 & 31 & 1 & & 12 & 130 & 6 & 2 & 196 \\
\hline 73. E. punctatissimus (SCHENCK, 1853) & 1 & 3 & & 2 & & 4 & 1 & & 11 \\
\hline 74. E. quadrinotatulus (SCHENCK, 1861) & & 1 & & & 5 & 2 & 1 & & 9 \\
\hline 75. E. rufitarsis (ZETTERSTEDT, 1838) & & 2 & & & 1 & 4 & & 1 & 8 \\
\hline 76. E. sexstrigatus (SCHENCK, 1868) & 22 & & 2 & 1 & 2 & 1 & & & 28 \\
\hline 77. E. tarsatus (SCHENCK, 1868) & 1 & & 1 & & & 1 & & & 3 \\
\hline 78. E. villosulus (KIRBY, 1802) & 1 & & & & & & & 8 & 9 \\
\hline 79. S. albilabris (FABRICIUS, 1793) & & & 1 & & & & & & 1 \\
\hline 80. S. crassus THOMSON, 1870 & 19 & 1 & 1 & & & & & 1 & 22 \\
\hline 81. S. ephippius (LinNAEUS, 1767) & 10 & 3 & 1 & & & 14 & 2 & 2 & 32 \\
\hline 82. S. gibbus (LINNAES, 1758) & & & & & & & & 2 & 2 \\
\hline 83. S. miniatus HAGENS, 1882 & 4 & & & & & 2 & 1 & & 7 \\
\hline 84. S. monilicornis (KIRBY, 1802) & 1 & & 1 & & & & & & 2 \\
\hline 85. S. pellucidus SмIтH, 1845 & 5 & & & & & 1 & 1 & & 7 \\
\hline 86. S. puncticeps THOMSON, 1870 & 1 & & 2 & & & 2 & & & 5 \\
\hline 87. S. reticulatus THOMSON, 1870 & 1 & & & & & & & & 1 \\
\hline \multicolumn{10}{|c|}{ Melittidae } \\
\hline 88. Melitta haemorrhoidalis (FABRICIUS, 1775) & & & 3 & & & & & & 3 \\
\hline
\end{tabular}


Table 4. Continued.

\begin{tabular}{|c|c|c|c|c|c|c|c|c|c|}
\hline Species & 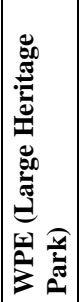 & 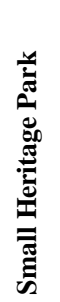 & 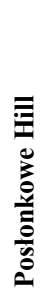 & 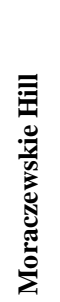 & 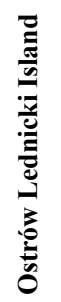 & 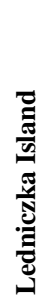 & 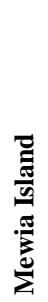 & $\frac{\mathscr{\omega}}{\tilde{\Xi}}$ & ڤ \\
\hline 89. Melitta leporina (PANZER, 1799) & 18 & & 1 & & & & & 3 & 22 \\
\hline 90. Macropis europaea WARNCKE, 1973 & & 2 & & & 1 & & 3 & & 6 \\
\hline 91. Dasypoda hirtipes (HARRIS, 1780) & 12 & & 4 & 1 & & & & 1 & 18 \\
\hline \multicolumn{10}{|c|}{ Megachilidae } \\
\hline 92. Trachusa byssina (PANZER, 1798) & & & 1 & & & & & & 1 \\
\hline 93. Anthidium manicatum (LINNAEUS, 1758) & 1 & & & & 10 & & & & 11 \\
\hline 94. Stelis breviuscula (NYLANDER, 1848) & 4 & & & & 1 & & & & 5 \\
\hline 95. S. phaeoptera (KIRBY, 1802) & 6 & & & & 2 & & & & 8 \\
\hline 96. S. punctulatissima (KIRBY, 1802) & 3 & & 1 & & & & & & 4 \\
\hline 97. Heriades crenulatus NYLANDER, 1856 & 6 & & & & & & & 2 & 8 \\
\hline 98. H. truncorum (LINNAEUS, 1758) & 13 & 2 & & & & & & 3 & 18 \\
\hline 99. Chelostoma florisomne (LINNAEUS, 1758) & 45 & 12 & & & & & & 1 & 58 \\
\hline 100. C. rapunculi (LEPELETIER, 1841) & & & 1 & & & & & & 1 \\
\hline 101. Anthocopa papaveris (LATREILLE, 1799) & 5 & & 18 & & & & & & 23 \\
\hline 102. Hoplitis adunca (PANZER, 1798) & & & 1 & & & & & & 1 \\
\hline 103. H. claviventris (THOMSON, 1872) & & & 1 & & & & & & 1 \\
\hline 104. Osmia aurulenta (PANZER, 1799) & & & 1 & & & & & & 1 \\
\hline 105. O. brevicornis (FABRICIUS, 1798) & 6 & & & & & 1 & & & 7 \\
\hline 106. O. coerulescens (LINNAEUS, 1758) & 15 & 2 & & & 1 & & & & 18 \\
\hline 107. O. fulviventris (PANZER, 1798) & 23 & 4 & & 2 & 11 & 2 & & 1 & 43 \\
\hline 108. O. leaiana (KIRBY, 1802) & 3 & & & & & & & & 3 \\
\hline 109. O. pilicornis SMITH, 1846 & 1 & & & & & & & & 1 \\
\hline 110. O. rufa (LiNNAEUS, 1758) & 206 & 52 & & 12 & 17 & 24 & 9 & 4 & 324 \\
\hline 111. Chalicodoma ericetorum (LEPELETIER, 1841) & & & 1 & & 2 & & & & 3 \\
\hline 112. Megachile alpicola ALFKEN, 1924 & & & 1 & & & & & & 1 \\
\hline 113. M. centuncularis (LINNAEUS, 1758) & & & 1 & & & & & & 1 \\
\hline 114. M. circumcincta (KIRBY, 1802) & 3 & & & & & & & 1 & 4 \\
\hline 115. M. ligniseca $(\mathrm{KIRBY}, 1802)$ & 1 & & 1 & & 1 & 2 & & & 5 \\
\hline 116. M. versicolor SMITH, 1844 & 6 & 1 & 2 & & 5 & 1 & 1 & 1 & 17 \\
\hline 117. Coelioxys inermis (KIRBY, 1802) & & & & & 1 & & & & 1 \\
\hline 118. C. mandibularis NYLANDER, 1848 & & & 1 & & & & & & 1 \\
\hline 119. C. quadridentata (LINNAEUS, 1758) & 2 & & 2 & & 1 & & & 2 & 7 \\
\hline \multicolumn{10}{|c|}{ Anthophoridae } \\
\hline 120. Anthophora furcata (PANZER, 1798) & 1 & & & & 2 & 4 & 1 & & 8 \\
\hline 121 A. plagiata (ILLIGER, 1806) & 2 & & & & & & & & 2 \\
\hline 122. A. plumipes (PALLAS, 1772) & 8 & 2 & & & 15 & 19 & 5 & 2 & 51 \\
\hline
\end{tabular}


Table 4. Continued.

\begin{tabular}{|c|c|c|c|c|c|c|c|c|c|}
\hline Species & 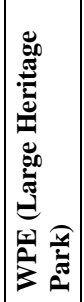 & 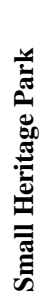 & 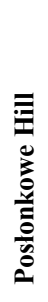 & 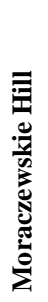 & 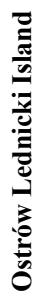 & 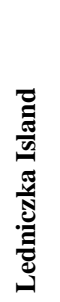 & 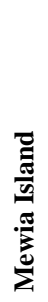 & $\frac{\mathscr{a}}{\bar{\Xi}}$ & ڤ \\
\hline 123. Melecta punctata (FABRICIUS, 1775) & 7 & 1 & & & 4 & 3 & & & 15 \\
\hline 124. Eucera longicornis (LINNAEUS, 1758) & 1 & & & & & & & & 1 \\
\hline 125. Ceratina cyanea (KIRBY, 1802) & 8 & & 2 & & & & & & 10 \\
\hline 126. Nomada bifasciata OLIVIER, 1811 & 2 & & & & & & & & 2 \\
\hline 127. N. fabriciana (LINNAEUS, 1767) & & & & & & & 1 & & 1 \\
\hline 128. N. facilis SCHWARZ, 1967 & & & 1 & & & & & & 1 \\
\hline 129. N. ferruginata (Linnaeus, 1767) & & 2 & & & 6 & 9 & 3 & & 20 \\
\hline 130. $\quad N$. flava PANZER, 1798 & & & & & & 1 & 2 & & 3 \\
\hline 131. N. flavoguttata (KIRBY, 1802) & & & & & & 1 & & & 1 \\
\hline 132. N. fucata PANZER, 1798 & 4 & & & & & & & & 4 \\
\hline 133. N. fulvicornis FABRICIUS, 1793 & 2 & & & & & 6 & & & 8 \\
\hline 134. N. goodeniana (KIRBY, 1802) & 3 & & & & & 2 & 2 & & 7 \\
\hline 135. N. lathburiana (KIRBY, 1802) & 5 & & & & & & & & 5 \\
\hline 136. N. leucophthalma (KIRBY, 1802) & & & & & 1 & & & & 1 \\
\hline 137. N. moeschleri ALFKEN, 1913 & & & & & & 1 & & & 1 \\
\hline 138. N. panzeri LEPELETIER, 1841 & 1 & & 1 & & & 1 & & 1 & 4 \\
\hline 139. N. ruficornis LINNAEUS, 1758 & 3 & & & & 6 & 9 & 10 & 1 & 29 \\
\hline 140. N. sheppardana (KIRBY, 1802) & 2 & & & & & & & & 2 \\
\hline 141. N. signata JURINE, 1807 & & & & & 2 & & 1 & 1 & 4 \\
\hline 142. N. striata FABRICIUS, 1793 & & & & & & 7 & & 1 & 8 \\
\hline 143. N. zonata PANZER, 1798 & & & & & & 5 & 1 & & 6 \\
\hline 144. Epeolus variegatus (LINNAEUS, 1758) & & & 1 & & & & & & 1 \\
\hline \multicolumn{10}{|c|}{ Apidae } \\
\hline 145. Bombus cryptarum FABRICIUS, 1775 & 3 & 1 & 1 & & 5 & & 1 & 1 & 12 \\
\hline 146. B. hortorum (LINNAEUS, 1761) & 9 & 1 & 2 & & 24 & 5 & 2 & 4 & 47 \\
\hline 147. B. hypnorum (LiNNAEUS, 1758) & 6 & 4 & 2 & & 17 & 13 & 5 & & 47 \\
\hline 148. B. jonellus (KIRBY, 1802) & & & & & & & 5 & & 5 \\
\hline 149. B. lapidarius (LiNNAEUS, 1758) & 25 & 5 & 5 & 3 & 24 & 5 & 1 & 9 & 77 \\
\hline 150. B. lucorum (LINNAEUS, 1761) & 38 & 10 & 2 & 4 & 17 & 27 & 21 & & 119 \\
\hline 151. B. muscorum (LINNAEUS, 1758) & 3 & 1 & & & 6 & 1 & 3 & & 14 \\
\hline 152. B. pascuorum (SCOPOLI, 1763) & 56 & 16 & 1 & 2 & 26 & 33 & 14 & 5 & 153 \\
\hline 153. B. pratorum (LINNAEUS, 1761) & 3 & & & & 9 & 19 & 1 & & 32 \\
\hline 154. B. ruderarius (MÜLLER, 1776) & 5 & & 4 & 4 & 20 & & & 2 & 35 \\
\hline 155. B. semenoviellus SKORIKOV, 1910 & & & 1 & & & 2 & 1 & & 4 \\
\hline 156. B. sylvarum (LINNAEUS, 1761) & 17 & 2 & 8 & 2 & 24 & 1 & 5 & 2 & 61 \\
\hline 157. B. terrestris (LINNAEUS, 1758) & 61 & 10 & 6 & 3 & 94 & 27 & 23 & 5 & 229 \\
\hline
\end{tabular}


Table 4. Continued.

\begin{tabular}{|c|c|c|c|c|c|c|c|c|c|c|}
\hline \multicolumn{2}{|r|}{ Species } & 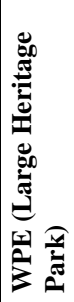 & 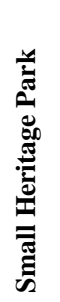 & 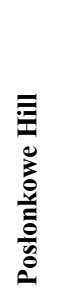 & 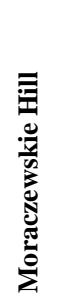 & 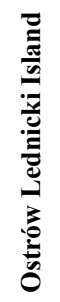 & 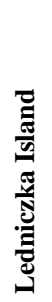 & 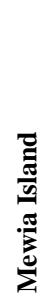 & t气 & $\stackrel{\Xi}{\emptyset}$ \\
\hline 158. & Bombus veteranus (FABRICIUS, 1793) & 1 & & 3 & & 10 & & & & 14 \\
\hline 159. & Psithyrus bohemicus (SEIDL, 1837) & 5 & 1 & 1 & & 4 & 2 & 2 & 2 & 17 \\
\hline 160. & P. rupestris (FABRICIUS, 1793) & 12 & 1 & 4 & & 18 & & & 3 & 38 \\
\hline 161. & Apis mellifera LinnaEus, 1758 & 177 & 68 & 1 & 48 & 20 & 98 & 49 & 17 & 478 \\
\hline & Sum & 1504 & 815 & 187 & 192 & 709 & 1142 & 472 & 138 & 5159 \\
\hline & Number of species & 111 & 65 & 68 & 34 & 69 & 80 & 57 & 47 & 161 \\
\hline
\end{tabular}

The number of species identified in particular habitats, and consequently, also the figure for the entire study area, is not complete, as the species accumulation curves for the three islands on Lake Lednica and the surrounding habitats never reached their asymptotes (Fig. 2). According to the Chao 2 estimator, up to 200 bee species can be expected to exist in the study area.

The differences in the numbers of species identified may be due to the different numbers of samples taken. The diversity of bee assemblages was comparable between sites, as evidenced by the similarity of the species accumulation curves with regard to samples. The Ostrów Lednicki Island bee assemblage appears to be a little more diverse and richer in species. However, the estimated curve for this site and the assemblages inhabiting the other two natural islands lie within one another's $95 \%$ confidence intervals; hence, these differences cannot be definitively treated as statistically significant. The bee assemblages at the mainland sites near Lake Lednica (habitat islands) were, as might be expected, more diverse and richer in species.

The number of species identified at individual sites varied from 34 to 111, depending on the duration of the fieldwork, sampling methods and diversity of the vegetation. Interestingly, technical differences (time, methods and, most importantly, the size of study sites) do not necessarily have a considerable effect on species diversity. For example, the small Ledniczka Island was found to support 70 species, compared to the 65 identified on Ostrów Lednicki Island, which is several times larger. The same number of species (65) was identified on Posłonkowe Hill, which is dominated by a relatively small patch of grassland; however, the immediate vicinity of that site (the adjoining wood, dirt roads and small agricultural areas) is very much more diversified. 


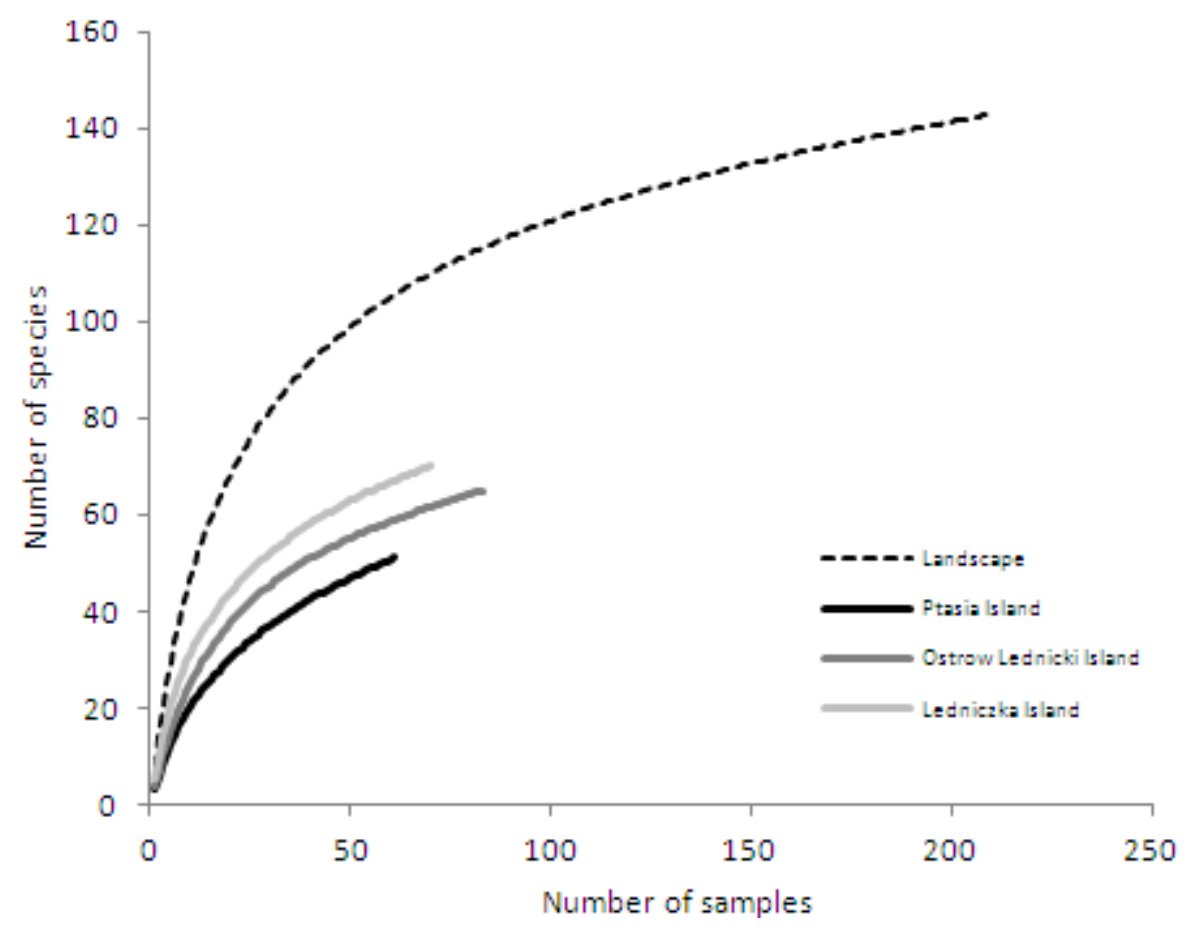

Fig. 2. Species accumulation curves.

The bee assemblage in the Ethnographic Park is characterised by the distinct predominance of the following four species, accounting for 7.5-8\% of the overall abundance of each (Fig. 3): Andrena haemorrhoa, A. helvola, Evylaeus calceatus and Osmia rufa. Collectively, they represent nearly a third $(28.7 \%)$ of the bee fauna at that site, with Bombus terrestris and Evylaeus pauxillus being the two subdominant species (approximately $10 \%$ of the total). The dominance patterns analysed at the individual sites are very different (Fig. 3). At some sites there are marked "chimney-like" dominance peaks of individual species; this is best exemplified by the Large Heritage Park site with its $16 \%$ contribution of Osmia rufa, or the significant proportion of Andrena helvola on Mewia Island (almost 30\%) and Ledniczka Island (almost 15\%). Ostrów Lednicki Island, in turn, had two species occurring very numerously, namely Evylaeus calceatus (over $15 \%$ of overall abundance) and Bombus terrestris, also with approximately $15 \%$ of the overall abundance of the assemblage there (Fig. 3). 

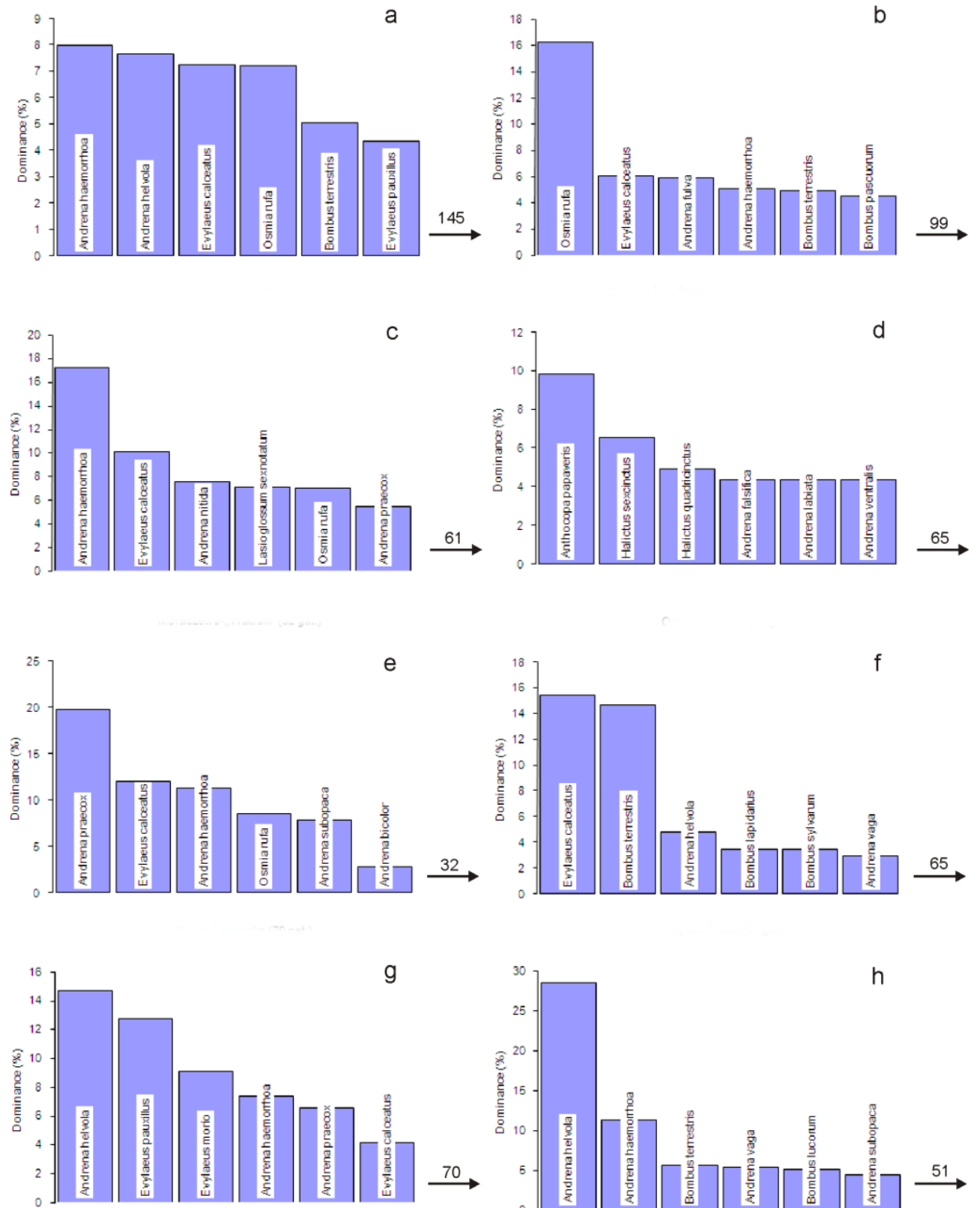

Fig. 3. Dominance patterns of Apiformes: $a$ - Lednicki Landscape Park, $b$ - Large Heritage Park, c - Small Heritage Park, d - Posłonkowe Hill, e - Moraczewskie Hill, f - Ostrów Lednicki island, $\mathrm{g}$ - Ledniczka island, $\mathrm{h}$ - Mawia Island. 


\section{Calculation of bee abundance (density)}

The bee density was calculated at two sites of the greatest importance for the bees in the study area, namely, the grassland in the Large Heritage Park (escarpment on the lake shore) and on Ostrów Lednicki Island, with its open habitats of meadows and pastures.

The overall bee abundance varied. The mean seasonal density of Apiformes during three years of fieldwork was more than twice as high on the island as on the mainland grassland. It was 2053.3 ind./ha on Ostrów Lednicki Island, compared to just 868.3 ind./ha on the escarpment in Large Heritage Park. The abundance of honeybees was similar at both sites - over 600 ind./ha. This indicates a fairly uniform distribution of Apis mellifera across the entire area.

Importantly, bee abundance varied by month. This was particularly apparent on the island, where the mean wild bee density in spring (April-May) was 1125 ind./ha, compared to the three times higher figure (3 325 ind./ha) in summer (July-August). The maximum wild bee density was $6750 \mathrm{ind}$./ha and 7200 ind./ha, recorded on 26 July 1995 and 9 August 1995, respectively. The density of bumblebees alone was 6200 ind./ha and 6450 ind./ha, respectively, on these two occasions.

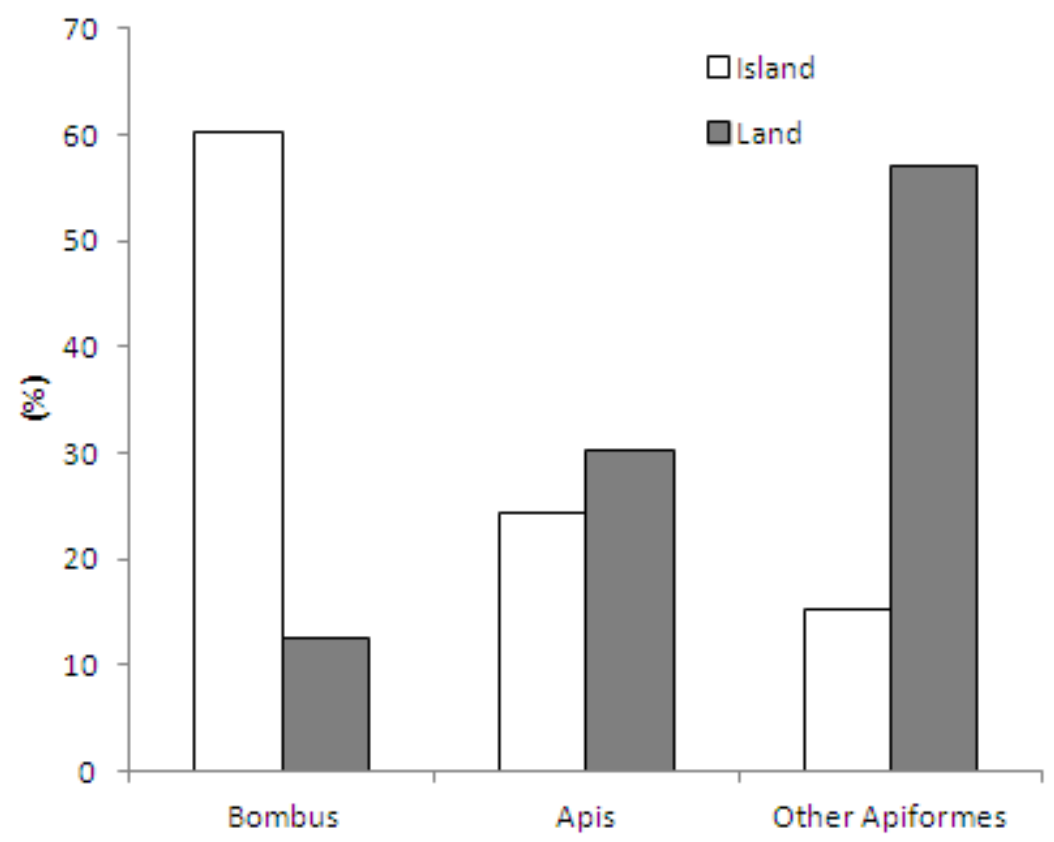

Fig. 4. Comparison of the share (\%) of honeybees, bumblebees and other Apiformes in the fauna on the islands of Lake Lednica and its surroundings. 
A more detailed analysis of the proportions of individuals groups of bees, i.e. the honeybee, bumblebees and the other wild bees, revealed significant differences between the mainland vicinity of the lake and the Ostrów Lednicki Island. The Ostrów Lednicki Island assemblage was characterised by a marked predominance of bumblebees $(60 \%)$, with a relatively low contribution from other wild Apiformes (about 15\%). At the mainland site, the escarpment at Dziekanowice, the quantitative relations were reversed, with wild bees accounting for almost $60 \%$ of the assemblage, and Bombus bees only slightly exceeding 10\% (Fig. 4).

\section{Aspects of bee phenology}

Very generally speaking, counts of 20-50 wild bee species were regularly obtained in a large part of the study area from mid-April to the second half of August. A marked rise was registered in May until the first 10 days of June. In the summer (July-August), species counts fell to 30-40.

The wild bee assemblages in the study area were characterised by a prominent contribution of spring species, as is well illustrated by the curves, which also show changes in the number of species during the growing season (Fig. 5).

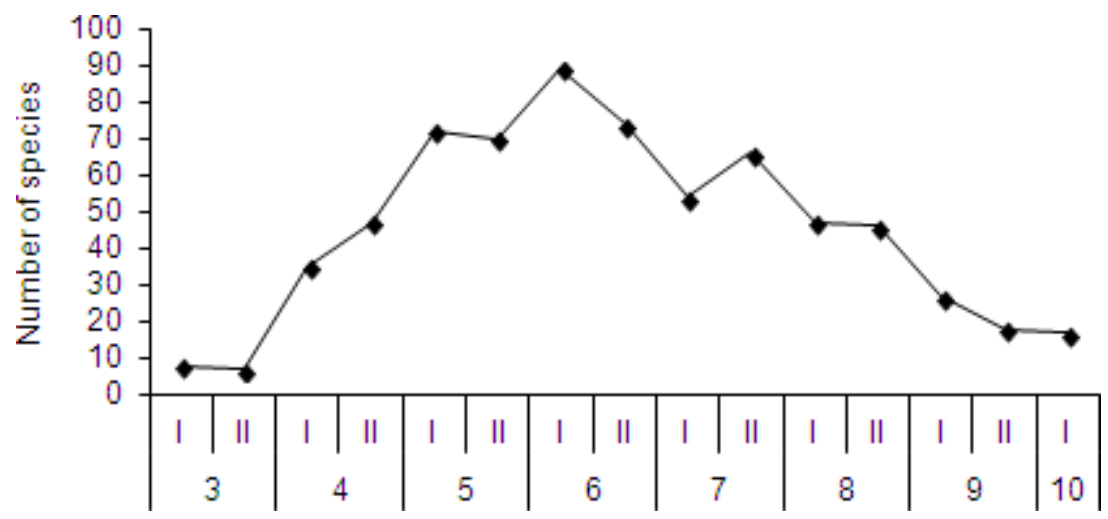

Fig. 5. Changes in the number of species of Apiformes during the growing season in the Lednicki Landscape Park.

The spring period mainly encompasses April and May, the time with the highest number of species recorded, although early bees (Andrena praecox, Bombus hypnorum and Lasioglossum sexnotatum) were seen already in the first half of March. Andrena vaga and Lasioglossum subfasciatum were recorded in the second half of March. Peak abundances for the entire study area were noted in the first half of May. 
Andrena and Bombus bees were found to strongly favour spring and they also ranked among the most abundant bees. Late April and early May were the time when large numbers of Andrena haemorrhoa, A. fulva, A. nitida, A. praecox, A. subopaca and A. vaga appeared. Other abundant early spring species included Evylaeus calceatus, E. morio, E. pauxillus, Osmia rufa and Anthophora plumipes. The most abundant bumblebee species at that time was Bombus terrestris.

Characteristic of the summer period (July-August) at the mainland sites was the constant presence of approximately 30-40 species. The most abundant species in that period included Evylaeus calceatus, E. morio and some bumblebee species, especially Bombus lucorum, B. lapidarius, B. pratorum and B. terrestris.

The dynamics of bee abundance was somewhat different at the island sites (Fig. 6), where bees were generally the most abundant in spring. The exception was Ostrów Lednicki Island, where there were two peaks of abundance, in May and July-August, a finding confirmed in each of the three years of fieldwork.
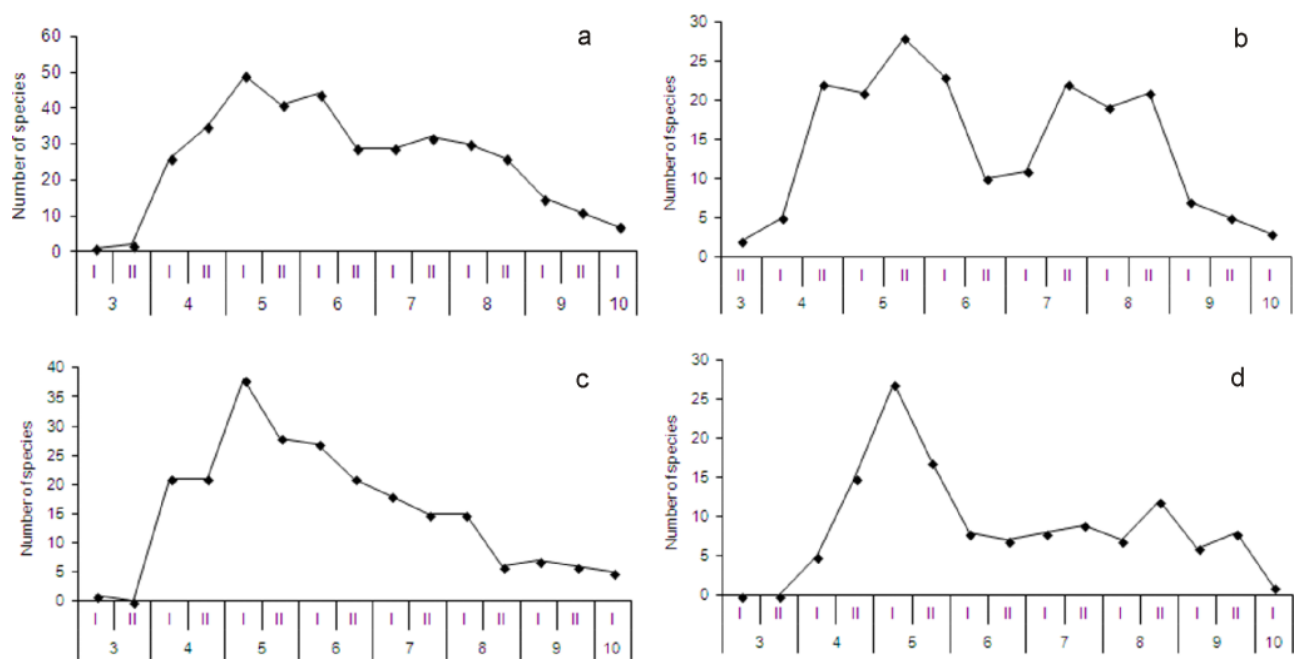

Fig. 6. Changes in the number of species of Apiformes in particular study sites in the Lednicki Landscape Park: a - surroundings of the lake, b - Ostrów Lednicki island, $\mathrm{c}$ - Ledniczka island, $\mathrm{d}$ - Mewia island.

The changes in the dynamics of bee appearance during the growing season were basically also confirmed by the changes in bee density on Ostrów Lednicki Island and on the grassland in the Large Heritage Park. The mainland site had a higher bee density in May (mean $1450 \mathrm{ind} . / \mathrm{ha}$ ), whereas the island site reached its peak abundance in summer, with 
the abundance of summer Apiformes being three times (3 325 ind./ha) as high as the figure obtained in spring.

\section{Uniqueness of the island bee fauna}

The description of the unique features of the island bee fauna was a major objective of the study. It appears that the study successfully identified differences between the islands and the mainland sites in the vicinity of the lake and also between particular islands, since the total of 161 species recorded in the Lednica Landscape Park includes species found all over the study area, i.e. both on the islands and at the mainland sites (more than half the taxa -89$)$. There is also a group of species found exclusively on the mainland (55 species) and a group recorded only on the islands (17 species).

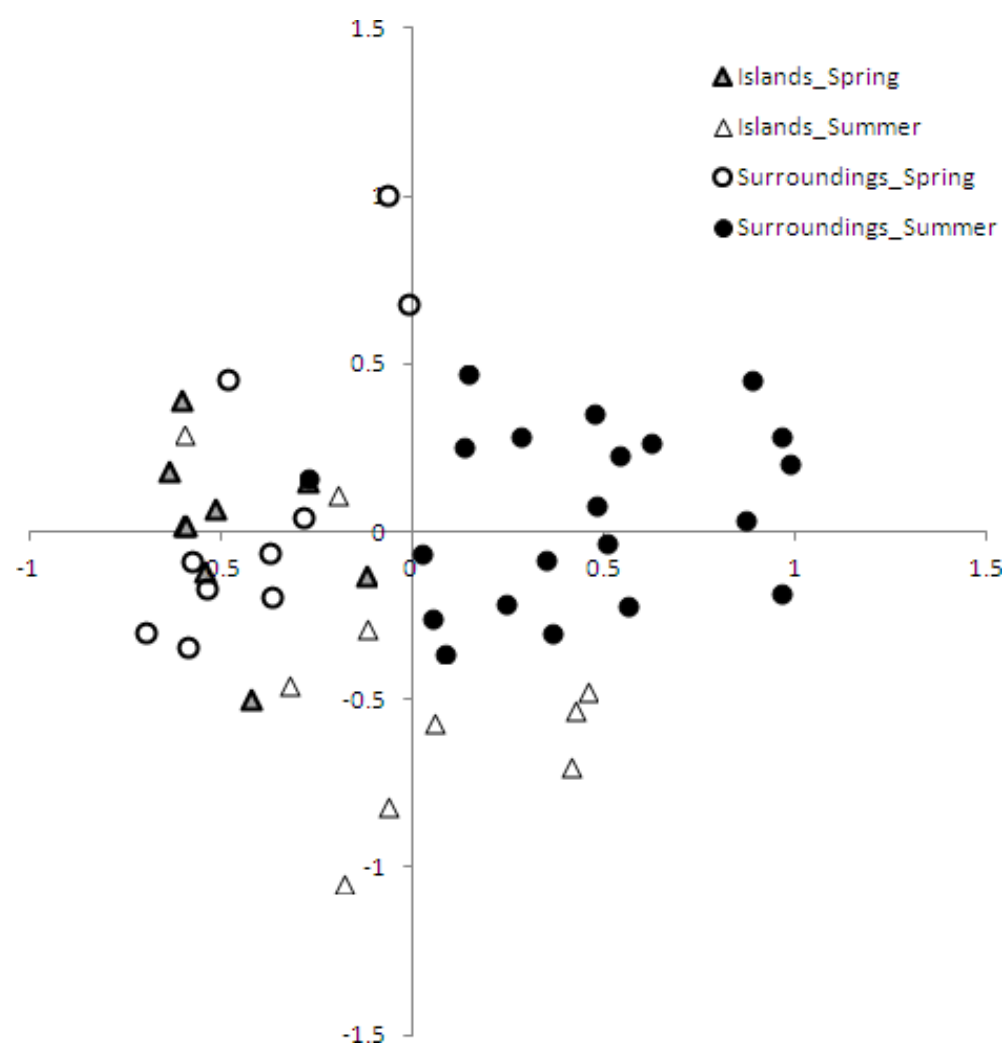

Fig. 7. Deagram of non-parametric ordination NMDS for assemblages of Apfirmes in studied sites in the LLP in particular years divided into spring and summer phenological group. A material collected in one year (III-VI and VI-X) was taken as a single sample. Bray-Curtis index was used as the distance. 
Table 4 shows that the three islands, differing in area and plant associations, also supported different numbers of species ( 80 vs. 69 vs. 57 ). It is hard to find a straightforward reason for this easy: the largest island, Ostrów Lednicki, did not have the highest number of bee species, even though it supported as many as 200 species of plants, compared to just 103 on Ledniczka Island and 80 on Mewia Island. It is therefore difficult to explain the finding of 80 bee species on Ledniczka Island. At the same time, Ostrów Lednicki Island supported an unique diversity of bumblebees, as 12 species were recorded there in abundances not seen at any other site. In early spring, when most specimens captured were females, density figures for bumblebees reached levels of 1600 ind./ha, while in summer maximum abundances of bumblebees reached 6500 ind./ha.

The bee assemblages from the islands and the mainland vicinity of Lake Lednica were analysed with regard to assemblage similarity. The seasonality of changes in assemblage indices was also accounted for, with all samples divided into a spring and summer phenological period (Fig. 7), i.e. containing bees collected between March and June (spring) and between June and October (summer), respectively. The wild bee assemblages in summer showed distinct structural differences both on the islands and on the mainland, forming distinct clusters. The spring assemblages appeared to be closer to one another, both on the mainland and on the islands. A general MRPP test showed up the significance of differences for four groups of samples $(\mathrm{P}<0.001)$. Pair-wise comparisons revealed significant differences for each pair compared $(\mathrm{P}<0.05)$. Overall, this means that the island and the mainland sites possessed unique species compositions and contributions of particular species in the two phenological periods. IndVal analysis revealed the species responsible for the significance of differences in the general MRPP test (Table 5).

Despite having access to similar food resources on the islands and on the mainland (a predominance of willows), the two clusters had different characteristic species. On the islands, the most characteristic species was Anthophora plumipes along with representatives of Andrena, such as Andrena helvola, A. vaga, A. praecox and A. tibialis. On the mainland, the dominant early spring species was Osmia rufa, which probably took advantage of the numerous wooden huts in both open-air museums, along with Colletes cunicularius and Andrena clarkella, both associated with sandy locations, as well as A. fulva, forming nesting colonies in light soil. In summer, the most characteristic species of the islands, in terms of abundance (as shown above) and diversity, are bumblebees, "headed" by Bombus lucorum, B. terrestris and B. hypnorum. The mainland assemblages in summer contain a different bee fauna, with no bumblebees among the indicator species, and characteristic forms including, most prominently, Heriades truncorum and Dasypoda hirtipes. 
Table 5. Characteristic species and their indicator values calculated according to the IndVal method for the four bee assemblages.

\begin{tabular}{|c|c|c|c|}
\hline Species & Group & IndVal & Probability \\
\hline Anthophora plumipes & \multirow{7}{*}{$\begin{array}{l}\text { Islands } \\
\text { Spring }\end{array}$} & 0.6838 & 0.001 \\
\hline Andrena helvola & & 0.6701 & 0.003 \\
\hline A. vaga & & 0.5737 & 0.001 \\
\hline Nomada ruficornis & & 0.4211 & 0.003 \\
\hline Andrena praecox & & 0.3989 & 0.012 \\
\hline Nomada ferruginata & & 0.3938 & 0.002 \\
\hline Andrena tibialis & & 0.3838 & 0.007 \\
\hline Bombus lucorum & \multirow{11}{*}{$\begin{array}{l}\text { Islands } \\
\text { Summer }\end{array}$} & 0.5921 & 0.002 \\
\hline B. terrestris & & 0.5682 & 0.004 \\
\hline B. hypnorum & & 0.5615 & 0.002 \\
\hline Anthophora furcata & & 0.5 & 0.001 \\
\hline Bombus pascuorum & & 0.4062 & 0.024 \\
\hline Evylaeus quadrinotatulus & & 0.3556 & 0.005 \\
\hline Bombus pratorum & & 0.3231 & 0.037 \\
\hline B. muscorum & & 0.312 & 0.025 \\
\hline Andrena nasuta & & 0.3 & 0.019 \\
\hline Evylaeus minutissimus & & 0.2812 & 0.025 \\
\hline Bombus veteranus & & 0.252 & 0.05 \\
\hline Osmia rufa & \multirow{10}{*}{$\begin{array}{l}\text { Surroundings } \\
\text { Spring }\end{array}$} & 0.6496 & 0.003 \\
\hline Andrena fulva & & 0.552 & 0.002 \\
\hline A. nigroaenea & & 0.4483 & 0.009 \\
\hline A. bicolor & & 0.3948 & 0.021 \\
\hline A. flavipes & & 0.3712 & 0.015 \\
\hline A. minutula & & 0.3496 & 0.019 \\
\hline A. apicata & & 0.3429 & 0.025 \\
\hline A. clarkella & & 0.3208 & 0.023 \\
\hline A. ventralis & & 0.3 & 0.025 \\
\hline Colletes cunicularius & & 0.2919 & 0.006 \\
\hline Heriades truncotum & \multirow{5}{*}{$\begin{array}{l}\text { Surroundings } \\
\text { Summer }\end{array}$} & 0.4762 & 0.003 \\
\hline Dasypoda hirtipes & & 0.4286 & 0.002 \\
\hline Halictus sexcinctus & & 0.2861 & 0.045 \\
\hline H. quadricinctus & & 0.2857 & 0.025 \\
\hline Lasioglossum leucozonium & & 0.2857 & 0.035 \\
\hline
\end{tabular}

\section{Associations between bees and plants}

Researchers of the flora of the Lednica Landscape Park have described 561 species of vascular plants (CELKA 1998), most of which are potential food plants of bees. Knowledge of these plants makes it easier to understand assemblage indices and interpret bee assemblages at the study sites, and particularly on the islands. The number of plant species 
indicates a great potential. Of special importance are the data from the islands, as the vegetation cover of Mewia Island had not been previously described. We know now that this small stretch of land supports a relatively rich flora of 80 species. A comparison of the sizes of bee populations on the islands shows that there is no clear-cut correlation between the area of an island and the number of plant species on the one hand and the number of species and abundance of bees on the other. The diversity of Apiformes turned out to be the highest not on Ostrów Lednicki Island, but on Ledniczka Island, an island several times smaller in size and supporting half as many plant species, mostly tree and shrub communities. This indicates that Ledniczka Island, 1.3 ha in area, rather than being an independent locality, acts mainly as a periodically rich source of food between the mainland (the village of Latalice) and the larger, neighbouring Ostrów Lednicki Island. The distance from the mainland is $100 \mathrm{~m}$ and from the neighbouring island is $200 \mathrm{~m}$. Such distances are easily negotiated even by small bees. The small Ledniczka Island supports such trees and shrubs as Salix alba, S. cinerea, S. fragilis, Prunus spinosa, P. domestica, Ribes spicatum, Rosa canina, $R$. rugosa and Rubus caesius, which offer rich sources of food for local and in-flying bees.

In contrast to the above statement is the finding of a large abundance of Evylaeus pauxillus in May and June and until mid-July. Interestingly, only single individuals were recorded at other sites, which may indicate that Ledniczka Island offers the best habitat conditions for this species.

Other bees numerously recorded from Ledniczka Island include representatives of Andrena, such as Andrena haemorrhoa, A. helvola and A. praecox, but these bees were also abundant at other sites. Ostrów Lednicki Island, in turn, exemplifies an independent island locality, especially with regard to the abundance of bumblebees, which clearly indicates the presence of rich food resources. The 200 plant species identified on Ostrów Lednicki Island (Table 1) provide diversified and ample food resources throughout the growing season, as confirmed by the presence of bumblebees at abundances not recorded at other sites.

Regardless of the potential food resources, insects, particularly social ones (including bumblebees), are attracted to patches of abundantly blooming plants. During such periods, they were found to occur quite numerously on a few abundantly blooming plants. For example, the most important food plants on Ostrów Lednicki Island in April and May were Lamium album, Taraxacum officinale and Primula veris, supporting bee densities up to 1700 ind./ha. After the meadows on the island had been mowed, bees switched to Juneblooming Vicia sepium and Anchusa officinalis, when densities of approx. 33-50 ind./ha were recorded. In July and August the bees moved on to Anchusa officinalis, Cirsium arvense and possibly also Ballota nigra, with densities then reaching nearly 7000 ind./ha.

In May, most bees fed on the flowers of Senecio vernalis, in densities of approx. 1100 ind./ha. In June the preferred flowers were those of Vicia sp. div., Anchusa officinalis, 
Hieracium pilosella, and in July and August mostly Medicago sp. div., Melilotus officinalis, Centaurea rhenana and Anchusa officinalis, with densities reaching 2750 ind./ha.

\section{DISCUSSION}

Of the various forms of spatial management seen in the region of Lake Lednica and Lednogóra, agriculture and the consequent dominance of arable land have the greatest impact on plant populations and communities, and, consequently, on the plant landscape (ChMiEL 1993, RATYŃSKa 2003). Accordingly, the localities investigated in the present study may be regarded as specific habitat islands that stand out from the background environment of arable fields or the waters of Lake Lednica. In accordance with the conception of FORMAN \& GODRON (1986), habitat islands are small patches within extensive matrices, sometimes connected through a network of ecological corridors.

The study sites vary with regard to their terrain patterns (from flat and undulating to a steep, high escarpment), potential natural vegetation (from aquatic and near-water habitats to alder forest, various types of riparian forest, oak-hornbeam forest, mixed coniferous forest, to mesic coniferous forest) and the forms and intensity of human impact (settlement, meadows and pastures, forest areas). These differences are reflected in the richness and diversity of the vegetation cover. Table 3 compares the study sites with respect to the gradient of decreasing human impact.

The study sites were found to support from 67 (Small Heritage Park) to 200 (Lednica) vascular plant species. Such large discrepancies stem from differences in size, habitat diversity and the diversity of human pressure in the study localities. Despite being subject to varying intensities of such pressure, all the study sites predominantly support native plant taxa, their contributions ranging from a little less than $52 \%$ (the gardens in the Large Heritage Park) to more than $90 \%$ (Mewia Island). The proportion of non-synanthropic spontaneophytes ranges from $9 \%$ in the gardens in the Large Heritage Park (the greatest degree of transformation) to almost $40 \%$ on Ledniczka Island and almost $44 \%$ on Mewia Island, where they are co-dominant with apophytes. Among anthropophytes, there is always a predominance of archaeophytes (range: 6.3-24.7\%), which is the result of the form of land management and the presence of neighbouring arable fields. CELKA (1998) suggests that the high proportion of earlier alien species in the flora of the former forts in the LLP $(12.7 \%)$ should be linked to past settlement, which continued for several centuries. Kenophytes reach their highest percentages in the gardens in the Large and Small Heritage Parks (over 15\% and almost 15\%, respectively), reflecting the cultivation of numerous nonnative plant species in these localities. 
Hemicryptophytes are the dominant life form at all the study sites; this is likewise the case for the entire Polish flora (cf. KoRNAŚ \& MEDWECKA-KoRNAŚ 1986, MATUSZKIEWICZ 1990). Their proportions ranged from almost 39\% (the gardens in the Large Heritage Park) to over $48 \%$ (Posłonkowe Hill). Therophytes constituted another significant group, with proportions from approximately 16\% (Mewia Island) to almost 32\% (the escarpment in the Large Heritage Park). This high contribution of therophytes indicates early successional stages. Importantly, the prevailing types of species among therophytes are synanthropic species, including both indigenous and anthropophytes (see e.g. JACKOWIAK 1990, 1998, CELKA 1999, RATYŃSKA 2003). Among phanerophytes, there was usually some predominance of shrubs.

The two phytosociological classes encompassing the largest number of species are Artemisietea and Molinio-Arrhenatheretea. The proportions of their representatives ranged from almost $15 \%$ to over $28 \%$ and from almost $15 \%$ to $25 \%$, respectively. At the most transformed sites (the Large and Small Heritage Parks), there is a definite numerical advantage of edificators of the Stellarietea mediae class. The highest proportions of thermophilous taxa of the Festuco-Brometea, Koelerio-Corynephoretea and FestucoBrometea classes were noted on the steep escarpments and hills (the escarpment in the Large Heritage Park, the windmills in Moraczewo and on Posłonkowe Hill). These species groups are typically associated with non-forested habitats. Forest-associated taxa of the Querco-Fagetea, Alnetea glutinosae and Salicetea purpureae classes and reed-associated ones of the Phragmitetea class are characteristic of the islands on Lake Lednica, i.e. habitats where the more natural vegetation cover has been preserved or secondary succession (regeneration) has reached an advanced stage.

Special-care species (protected and endangered) were found at nearly all the study sites. The largest number of such species (4-5) was found on the escarpment in the Large Heritage Park and on Lednica. This indicates a significant role of semi-natural ecosystems in protecting the gene pool of protected and endangered plants.

The proportion of indigenous communities ranged from $30 \%$ (the gardens in the Large Heritage Park) to over 90\% (Mewia Island). The islands on Lake Lednica are characterised by the presence of natural perdochoric phytocoenons. Their occurrence was correlated with the presence of forest-related species and indicates a high degree of naturalness of the study area at an advanced stage of secondary succession. The presence of ruderal communities at all the study sites is evidence of more or less intense, current or past human impact.

We studied the main bee refuges within the Park by establishing seven permanent study sites. They were located in the immediate vicinity of Lake Lednica (sites 1-4) and on the three islands. The sites represent mainly permanent meadows and pastures, and particularly grasslands and near-water habitats. We did not investigate areas subjected to intensive agricultural use, which are dominant in the LLP. This area, where mineral fertilisers and 
plant protection agents have been used unsparingly (TOBOLSKI 1991), was largely deforested a thousand years ago. There is a small forested area (secondary stands of pine) at the northern end of Lake Lednica.

Research conducted over many years has revealed the presence of 161 species of Apiformes, corresponding to nearly a third of all the bee species found in Poland; however, up to 200 bee species may be expected to live there. The species count in the LLP is similar to the number identified in the Mazurian Landscape Park or in the various national parks in Poland, despite the striking differences in the size or percentage of forested areas. A study of field afforestation and roadside grasslands, representing bee refuges, in the similarly predominantly agricultural area of the Gen. D. Chłapowski Agroecological Landscape Park in the Leszno Lakeland (Pojezierze Leszczyńskie), identified 75 species (BANASZAK 1983).

Table 6. Comparison of the number of bee species recorded in selected Polish Lowland national parks and in the LLP.

\begin{tabular}{|c|c|c|c|c|c|}
\hline Park & $\begin{array}{c}\text { Area } \\
\text { (ha) }\end{array}$ & $\begin{array}{c}\text { Forest area } \\
(\%)\end{array}$ & $\begin{array}{c}\text { No. of bee } \\
\text { species }\end{array}$ & $\begin{array}{c}\text { \% of Polish } \\
\text { fauna }\end{array}$ & Authors \\
\hline Białowieża NP & 10517 & 96.0 & 181 & 38.6 & $\begin{array}{c}\text { BISCHOFF (1925), BANASZAK } \\
\text { \& JAROSZEWICZ (2009) }\end{array}$ \\
\hline $\begin{array}{c}\text { NP Bory } \\
\text { Tucholskie }\end{array}$ & 4798 & 79.0 & 101 & 21.1 & $\begin{array}{c}\text { BANASZAK \& WENDZONKA } \\
(2002)\end{array}$ \\
\hline Drawa NP & 11342 & 82.0 & 121 & 25.5 & BANASZAK et al. (2009) \\
\hline Wigry NP & 15085 & 62.7 & 191 & 40.7 & $\begin{array}{c}\text { BANASZAK \& KRZYSZTOFIAK } \\
(1996)\end{array}$ \\
\hline $\begin{array}{c}\text { Wielkopolski } \\
\text { NP }\end{array}$ & 7620 & 58.3 & 226 & 48.2 & $\begin{array}{c}\text { BANASZAK 1987, BANASZAK } \\
\text { \& CIERZNIAK (1994), } \\
\text { CIERZNIAK (2003) }\end{array}$ \\
\hline Wolin NP & 10937 & 41.0 & 133 & 28.0 & BANASZAK (2010) \\
\hline Masurian LP & 53665 & 50.0 & 153 & 32.6 & BANASZAK (2010) \\
\hline Narew NP & 7350 & 12.0 & 125 & 26.4 & BANASZAK (2006) \\
\hline Kampinoski NP & 38544 & 75.0 & 180 & 38.2 & BANASZAK \& PLEWKA (1981) \\
\hline Lednica LP & 4854 & 9,1 & 161 & 34.0 & new daa \\
\hline
\end{tabular}

A similar species count in the same landscape (Gen. D. Chłapowski Agroecological Landscape Park) was obtained by CIERZNIAK (1995). Table 6 compares various data on bee diversity in 10 national parks and landscape parks of different sizes and proportions of afforested land. However, there is no clear, direct correlation between these indices and the overall numbers of bee species. Even if we assume that the figures in the table (assumed to represent $80 \%$ ) are different from the actual numbers of species, one should bear in mind that the examples of various landscape types of diverse sizes (from approximately 5 to 10 or 50 thousand hectares) listed in the table are home to approximately 30 to $50 \%$ of the bee 
fauna in Poland. The possibility of a link between the small size of a habitat and species richness is indicated by the data from the islands on Lake Lednica, where the small Ledniczka Island (1.3 ha) supported more species than the much larger Ostrów Lednicki Island (7.5 ha). Similarly, studies of habitat islands near Bydgoszcz (mineral hills around hay and periodically inundated meadows) occupying similarly small areas (3 ha and 6 ha) found 89 species (BANASZAK et al. 2004) and 108 species (BANASZAK et al. in press), respectively. In the extensive Bory Tucholskie National Park, where a study was conducted in the sparse, open enclaves among the forests, only 101 bee species were identified. These problems, not adequately studied to date, demand more detailed and insightful research.

Floristic research has shown that the food resources for bees in the area we studied comprised mainly xerothermal grasslands on escarpments and willow thickets immediately adjacent to Lake Lednica and on the islands. These groups of habitats (Table 2) are permanent and act as life refuges and locations of food plants in arable fields and their margins. The great importance of willow thickets is indicated by the large diversity of bees, especially early spring Andrena bees of the subgenus Andrena s. str., where the dominants were Andrena haemorrhoa, A. helvola and A. praecox, feeding on many flowers of Salix species, as this habitat supports all but three Polish species of Andrena s. str. Similar findings were obtained in the Narew National Park where, thanks to the dominant willow thickets, nearly all the Polish species of the subgenus Andrena can be found (BANASZAK 2006). In turn, the great diversity of the vegetation in terms of moisture (from reed beds and willow thickets to mesic meadows) on Ostrów Lednicki Island provides very favourable conditions for bumblebees. While the island is a nesting place for bumblebees, these bees also visit it during the blooming of attractive plants, resulting in bumblebee densities not noted anywhere else.

The high diversity of pollinating insects on islands supporting natural phytocoenoses is a contemporary reflection of the natural environment from the past, even though human beings have been ever present there for a thousand years.

\section{REFERENCES}

BANASZAK J. 1980. Studies on methods of censusing the numbers of bees (Hymenoptera, Apoidea). Polish Ecological Studies 6(2): 355-366.

BANASZAK J. 1983. Ecology of bees (Apoidea) of agricultural landscape. Polish Ecological Studies 9(4): 421-505.

BanaszaK J. 1995. Old buildings as refugia of life of Hymenoptera. Chrońmy Przyrodę Ojczystą 51(4): 94-97. (in Polish)

BANASZAK J. 1996. Preliminary results of studies on natural resources of pollinating insects in Lednicki Landscape Park. Studia Lednickie 4: 495-498. (in Polish) 
BANASZAK J. 1998. Old rural buildings - as a place of life of Hymenoptera. Studia Lednickie 5: 293-305. (in Polish)

BANASZAK J. 2000. Studies on insect fauna of Lednicki Landscape Park. Preliminary report. Studia Lednickie 6: 417-422. (in Polish)

BANASZAK J. 2006. Bees (Hymenoptera: Apiformes) in the Narew National Park. Polish Journal of Entomology 75(4): 511-537.

BANASZAK J., RATYŃSKa H., BANASZAK W.A. 2004. Proposed "Folusz" Nature Reserve near Szubin (in Poland) as a refuge of thermophilous plant cover and the fauna of the superfamilies Apoidea and Scolioidea. Badania Fizjograficzne nad Polską Zachodnią, seria C - Zoologia 50: 101-132. (in Polish)

BANASZAK J., RATYŃSKa H., SZEFER P. in press. Diversity of bees in relation to plant cover. The case of "Ostrów Małe Rudy" habitat island in the Noteć River valley near Bydgoszcz (Hymenoptera: Apoidea, Apiformes). Badania Fizjograficzne.

BANASZAK W.A. 2005. What has caused the decline of the solitary bee Anthophora plagiata (ILLIGER, 1806) (Hymenoptera: Apoidea) in the Wielkopolska-Kujawy Lowland in west Poland? Polish Journal of Entomology 74(2): 157-185.

Barkman J.J., Doing H. \& Segal S. 1964. Kritische Bemerkungen und Vorschläge zur quantitativen Vegetationsanalyse. Acta Botanica Neerlandica 13(3): 394-419.

Bednarek R., Prusinkiewicz Z. 1997. Geography of soils. PWN, Warszawa. (in Polish)

BonNer J.L., ANDERSON J.T., RENTCh J.S., GRAFOn W.N. 2009. Vegetative composition and community structure associated with beaver ponds in Canaan valley, West Virginia, USA. Wetlands Ecology and Management 17(5): 543-554.

BuCZeK K. 1960. Works of the Historical Commission, 5. Polish lands a thousand years ago (a geographical and historical outline). Wydawnictwo PAN, Wrocław - Kraków. (in Polish)

BURNHAM K.P., Overton W.S. 1979. Robust estimation of population size when capture probabilities vary among animals. Ecology 60(5): 927-936.

CELKa Z. 1998. Vascular plants of settlements in Lednicki Landscape Park. Studia Lednickie 5: 241-267. (in Polish)

CELKA Z. 1999. Works of the Department of Plant Taxonomy of Adam Mickiewicz University in Poznań, No. 9. Vascular plants of settlements in Gerat Poland. Bogucki Wydawnictwo Naukowe, Poznań. (in Polish)

Chmiel J. 1993. Works of the Department of Plant Taxonomy of Adam Mickiewicz University in Poznań, No. 1. Flora of vascular plants in the eastern part of Gniezno Lake District and its anthropogenic changes in the $19^{\text {th }}$ and $20^{\text {th }}$ centuries. Part 1 . Wydawnictwo Sorus, Poznań. (in Polish)

Chojnacka M., RaszKa B. 2007. Lednicki Landscape Park. Wydawnictwo WBPiCAK, Poznań. (in Polish)

CIERZNIAK T. 1995. The effect of a differentiation of agricultural landscape on Apoidea communities. Zeszyty Naukowe Wyższej Szkoły Pedagogicznej w Bydgoszczy, Studia Przyrodnicze 11: 13-50.

Colwell R. K. 2006. EstimateS: Statistical estimation of species richness and shared species from sample. Version 8.0.0. User's Guide and applications. Internet: http://purl.oclc.org/estimates 
Colwell R.K., Mao C.X., Chang J. 2004. Interpolating Extrapolating, and Comparing Incidence based Species Accumulation Curves. Ecology 85(10): 2717-2727.

Drzymata S., Mocek A. 1994. The suitability of soils in Lednica Landscape Park in the aspect of water protection and landscape. Studia Lednickie 3: 309-326. (in Polish)

DUfRÊNE M., LEGENDRE P., 1997. Species assemblages and indicator species: the need for a flexible asymmetrical approach. Ecological Monographs 67(3): 345-366.

FAITH D.P., Minchin P.R., BelBin L. 1987. Compositional dissimilarity as a robust measure of ecological distance. Vegetatio 69(1): 57-68.

Forman R.T.T., Godron M. 1986. Landscape Ecology. Wiley and Sons, New York.

Gotelli N.J., Colwell R.K. 2001. Quantifying biodiversity: procedures and pitfalls in measurement and comparison of species richness. Ecology Letters 4(4): 379-391.

Hensel W., HilcZer-KurnatowsKa Z. 1972. Studies on settlement in Wielkopolska in the early historical period. Vol. 4. Ossolineum, Wrocław - Warszawa - Kraków - Gdańsk. (in Polish)

HŁADYŁowiCZ K.J. 1932. Changes to the landscape and the development of settlement in Wielkopolska from the $14^{\text {th }}$ to the $10^{\text {th }}$ century. The study of social and economic history 12 . Kasa im. Rektora J. Mianowskiego, Instytut Popierania Polskiej Twórczości Naukowej, Lwów Warszawa. (in Polish)

JACKOWIAK B. 1990. Anthropogenic changes of the flora of vascular plants of Poznań. Wydawnictwo Naukowe Uniwersytetu Adama Mickiewicza w Poznaniu, Poznań. (in Polish)

JACKOWIAK B. 1998. Works of the Department of Plant Taxonomy of Adam Mickiewicz University in Poznań, No. 8. Spatial structure of urban flora. A methodological-cognitive study. Bogucki Wydawnictwo Naukowe, Poznań. (in Polish)

JaCkowiak B., Celka Z., Chmiel J., Żukowski W. 2007. Red list of vascular flora of Wielkopolska (Poland). Biodiversity: Research and Conservation 5-8(3-4): 95-127.

KANIECKI A. 1989. Commentary on the hydrographic map in scale 1:50.000, sheet no. 423.1. Okręgowe Przedsiębiorstwo Geodezyjno-Kartograficzne w Poznaniu, Poznań. (in Polish)

KondRaCKI J. 1998. Physical geography of Poland. Wydawnictwo Naukowe PWN, Warszawa. (in Polish)

Kornaś J., Medwecka-Kornaś A. 1986. Geography of plants. PWN, Warszawa. (in Polish)

KuRAntowska Z., KuRANTOWSKi S. 1991. Settlement of the region of Lednica in prehistoric times and the Middle Ages in light of previous research. [in:] K. TовOLSKI (ed.). Introduction to the paleoecology of the Lednicki Landscape Park. Wydawnictwo Naukowe UAM, Poznań, 35-42. (in Polish)

Matuszkiewicz W. 1990. Geobotanical regionalization. [in:] T. Trampler, A. Kliczkowska, E. DMYterko, A. SierpińsKA (eds.). Regionalization of natural-forest on the basis of ecological and physiographic. PWRiL, Warszawa, 134-157. (in Polish)

MiLESKA M.I. (ed.) 1983. Geographical and touring lexicon of Poland. PWN, Warszawa. (in Polish)

Oksanen J., Blanchet F.G., Kindt R., Legendre P., O'Hara R.B., Simpson G.L., Solymos P., StEvens M.H.H., WAGNER H. 2011. vegan: Community Ecology Package. R package version 1.17-11. Internet: https://cran.r-project.org/web/packages/vegan/index.html 
PolCYN M. 2000. Some remarks on the Early medieval plant communities around Ostrów Lednicki and Giecz. Studia Lednickie 6: 339-350. (in Polish)

RATYŃSKA H. 2003. Vegetation as an expression of anthropogenic landscape transformation on the example of the basin of the River Główna. Wydawnictwo Akademii Bydgoskiej im. Kazimierza Wielkiego, Bydgoszcz. (in Polish)

RatyŃska H., Wojterska M., Brzeg A., Kolacz M. 2010. Multimedia encyclopedia of plant communities in Poland, Ver. 1.1. NFOSiGW, UKW, IETI, Bydgoszcz. (in Polish)

RUtKOWSKi L., RATYŃSKa H., SzWED W. 1994. Vascular plants. List of taxa of plants of Poland intended to form databases geobotanical. [in:] S. HenNEKENS 1991-94. Turboveg (versie 9.23). IBN Wageningen, Wageningen.

RDCT [R Development Core Team] 2008. R: A language and environment for statistical computing. R Foundation for Statistical Computing. Vienna, Austria. Internet:: http://www.R-project.org

RoberTs D.W. 2010. labdsv: Ordination and Multivariate Analysis for Ecology. R package version 1.4-1. Internet: https://cran.r-project.org/web/packages/labdsv/index.html

Solon J. 1983. The local complex of phytocenoses and the vegetation landscape - fundamental units of the spatial organization of the vegetation above the phytocenose level. Acta Botanica Hungarica 29(1-4): 337-384.

TOBOLSKI K. 1996. The state of knowledge of nature in the Lednica Landscape Park and proposed forms of nature conservation. Studia Lednickie 4: 48-57. (in Polish)

ToBOLSKI K. 1991: The current state of palaeobotanical and biostratigraphic studies in the Lednicki Landscape Park. [in:] K. TOBOLSKI (ed.). Introduction to the paleoecology of the Lednicki Landscape Park. Wydawnictwo Naukowe UAM, Poznań, 11-34. (in Polish)

TÜXEN R. 1973: Vorschläge zur Aufnahme von Gesellschaftskomplexen in potentiell natürlichen Vegetationsgebieten. Acta Botanica Hungarica 19(1-4): 379-384.

Woś A. 1999: Climate of Poland. Wydawnictwo Naukowe PWN, Warszawa. (in Polish)

Woś A. 2003: Climate of the climatic region of Central Great Poland in the light of the incidence of selected types of weather and their variability in the years 1951-2000. [in:] J. BANASZAK (ed.). The Transformation of Great Poland Into a Steppe Region half a century later. Wydawnictwo Akademii Bydgoskiej im. Kazimierza Wielkiego, Bydgoszcz, 27-42. (in Polish)

Zimmerman G.M., Goetz H., MielKe Jr. P.W. 1985: Use of an improved statistical method for group comparisons to study effects of prairie fire. Ecology 66(2): 606-611.

Received: 1 October 2015

Accepted: 15 October 2015 